\title{
The 2D Hubbard model on the honeycomb lattice
}

\author{
Alessandro Giuliani \\ Dipartimento di Matematica, Università di Roma Tre, \\ L.go S. Leonardo Murialdo 1, 00146 Roma Italy \\ Vieri Mastropietro \\ Dipartimento di Matematica, Università di Roma Tor Vergata, \\ V.le della Ricerca Scientifica, 00133 Roma Italy
}

\begin{abstract}
We consider the 2D Hubbard model on the honeycomb lattice, as a model for a single layer graphene sheet in the presence of screened Coulomb interactions. At half filling and weak enough coupling, we compute the free energy, the ground state energy and we construct the correlation functions up to zero temperature in terms of convergent series; analiticity is proved by making use of constructive fermionic renormalization group methods. We show that the interaction produces a modification of the Fermi velocity and of the wave function renormalization without changing the asymptotic infrared properties of the model with respect to the unperturbed non-interacting case; this rules out the possibility of superconducting or magnetic instabilities in the ground state. We also prove that the correlations verify a Ward Identity similar to the one for massless Dirac fermions, up to asymptotically negligible corrections and a renormalization of the charge velocity.
\end{abstract}

\section{INTRODUCTION}

The recent experimental realization of a monocrystalline graphitic film, known as graphene 20], revived the interest in the low temperature physics of two-dimensional electron systems on the honeycomb lattice, which is the typical underlying structure displayed by single-layer graphene sheets. Graphene is quite different from most conventional quasi-two dimensional electron gases, because of the peculiar quasi-particles dispersion relation, which closely resembles the one of massless Dirac fermions in $2+1$ dimensions. This was already pointed out in [25] and further exploited in [24], where the analogy between graphene and 2+1-dimensional quantum electrodynamics (QED) was made explicit, and used to predict a condensed-matter analogue of the axial anomaly in QED. From this point of view, graphene can be considered as a sort of testing bench to investigate the properties of infrared QED in $2+1$ dimensions. Recently, the experimental observation of graphene greatly enhanced the study of the anomalous effects induced by the pseudo-relativistic dispersion relation of its quasi particles, see [6] for an up-to-date description of the state of art. Among the most unusual and exciting phenomena displayed by graphene, and already experimentally observed, let us mention the anomalous integer quantum Hall effect and the insensitivity to localization effects generated by disorder. It is reasonable to guess that the unique properties of graphene will have in the next few years several important applications in condensed matter and in nano-technologies.

The main reason behind these anomalous effects lies in the geometry of the Fermi surface, which at half filling is not given by a curve, as in usual 2D Fermi systems, but is completely degenerate: it consists of two isolated points, as in one dimensional Fermi systems. From a theoretical point of view, this fact completely changes the infrared scaling properties of the propagator. It has been pointed out, see for instance [11] and references therein, that, in the case of short-range electron-electron interactions, all the operators with four or more fermionic fields are irrelevant in a Renormalization Group (RG) sense; this suggests that the interaction should not affect too much the asymptotic behavior of the model, at least at small coupling. It should be remarked however that such RG analyses were performed only at a perturbative level, without any control on the convergence of the expansion, and directly in the relativistic approximation, consisting in replacing the actual dispersion relation by its linear approximation around the singularity; such approximation implies in particular the validity of a continuous Lorentz $U(1)$ symmetry that is not present in the original model.

Aim of this paper is to present the first rigorous construction of the low temperature and ground state properties of the 2D Hubbard model on the honeycomb lattice with weak local interactions; this is achieved by rewriting the correlation functions in terms of resummed series, 
convergent uniformly in the temperature up to zero temperature, as we prove by making use of constructive fermionic renormalization group. We show that indeed the interaction does not change the asymptotic infrared properties of the model with respect to the unperturbed non-interacting case, but it produces a renormalization of the Fermi velocity and of the wave function (note that no renormalization of the Fermi surface would be present in the relativistic approximation). Our result rules out the presence of superconducting or magnetic instabilities at weak coupling; this is in striking contrast with the Hubbard model on the square lattice, where quantum instabilities (corresponding to the magnetic or superconducting long range order that are presumably present in the ground state) prevent the convergence of the perturbative expansion in $U$ for low enough temperatures. We also prove that indeed the $2 \mathrm{D}$ Hubbard model on a honeycomb lattice is asymptotically described by a $\mathrm{QED}_{2+1}$ in the presence of an ultraviolet cutoff, massive "photons" and massless electrons; however the bare parameters of the QED theory must be carefully chosen to include lattice effects.

\section{THE MODEL AND THE MAIN RESULTS}

\section{A. The model}

The grandcanonical Hamiltonian of the 2D Hubbard model on the honeycomb lattice at half filling in second quantized form is given by:

$$
\begin{aligned}
H_{\Lambda} & =-\sum_{\substack{\vec{x} \in \Lambda \\
i=1,2,3}} \sum_{\sigma=\uparrow \downarrow}\left(a_{\vec{x}, \sigma}^{+} b_{\vec{x}+\vec{\delta}_{i}, \sigma}^{-}+b_{\vec{x}+\vec{\delta}_{i}, \sigma}^{+} a_{\vec{x}, \sigma}^{-}\right)+ \\
& +\frac{U}{3} \sum_{\substack{\vec{x} \in \Lambda \\
i=1,2,3}}\left[\left(a_{\vec{x}, \uparrow}^{+} a_{\vec{x}, \uparrow}^{-}-\frac{1}{2}\right)\left(a_{\vec{x}, \downarrow}^{+} a_{\vec{x}, \downarrow}^{-}-\frac{1}{2}\right)+\left(b_{\vec{x}+\vec{\delta}_{i}, \uparrow}^{+} b_{\vec{x}+\vec{\delta}_{i}, \uparrow}^{-}-\frac{1}{2}\right)\left(b_{\vec{x}+\vec{\delta}_{i}, \downarrow}^{+} b_{\vec{x}+\vec{\delta}_{i}, \downarrow}^{-}-\frac{1}{2}\right)\right]
\end{aligned}
$$

where:

1. $\Lambda$ is a periodic triangular lattice, defined as $\Lambda=\mathbb{B} / L \mathbb{B}$, where $L \in \mathbb{N}$ and $\mathbb{B}$ is the triangular lattice with basis $\vec{a}_{1}=\frac{1}{2}(3, \sqrt{3}), \vec{a}_{2}=\frac{1}{2}(3,-\sqrt{3})$.

2. The vectors $\vec{\delta}_{i}$ are defined as

$$
\vec{\delta}_{1}=(1,0), \quad \vec{\delta}_{2}=\frac{1}{2}(-1, \sqrt{3}), \quad \vec{\delta}_{3}=\frac{1}{2}(-1,-\sqrt{3})
$$

3. $a_{\vec{x}, \sigma}^{ \pm}$are creation or annihilation fermionic operators with spin index $\sigma=\uparrow \downarrow$ and site index $\vec{x} \in \Lambda$, satisfying periodic boundary conditions in $\vec{x}$

4. $b_{\vec{x}+\vec{\delta}_{i}, \sigma}^{ \pm}$are creation or annihilation fermionic operators with spin index $\sigma=\uparrow \downarrow$ and site index $\vec{x}+\vec{\delta}_{i} \in \Lambda+\vec{\delta}_{1}$, satisfying periodic boundary conditions in $\vec{x}$.

5. $U$ is the strength of the on-site density-density interaction; it can be either positive or negative.

Note that the Hamiltonian (2.1) is hole-particle symmetric, i.e., it is invariant under the exchange $a_{\vec{x}, \sigma}^{ \pm} \longleftrightarrow a_{\vec{x}, \sigma}^{\mp}, \quad b_{\vec{x}+\vec{\delta}_{1}, \sigma}^{ \pm} \longleftrightarrow-b_{\vec{x}+\vec{\delta}_{1}, \sigma}^{\mp}$ This invariance implies in particular that, if we define the average density of the system to be $\rho=(2|\Lambda|)^{-1}\langle N\rangle_{\beta, \Lambda}$, with $N=\sum_{\vec{x}, \sigma}\left(a_{\vec{x}, \sigma}^{+} a_{\vec{x}, \sigma}^{-}+b_{\vec{x}+\vec{\delta}_{1}, \sigma}^{+} b_{\vec{x}+\vec{\delta}_{1}, \sigma}^{-}\right)$the total particle number operator and $\langle\cdot\rangle_{\beta, \Lambda}=\operatorname{Tr}\left\{e^{-\beta H_{\Lambda}} \cdot\right\} / \operatorname{Tr}\left\{e^{-\beta H_{\Lambda}}\right\}$ the average with respect to the (grandcanonical) Gibbs measure at inverse temperature $\beta$, one has $\rho \equiv 1$, for any $|\Lambda|$ and any $\beta$. We also recall that a theorem [15] guarantees that at half filling the ground state of (2.1) is unique and its total spin is equal to zero.

Our goal is to characterize the low and zero temperature properties of the system described by (2.1), by computing thermodynamic functions (e.g., specific free energy and specific ground 
state energy) and a complete set of correlations at low or zero temperatures. To this purpose it is convenient to introduce the notions of specific free energy

$$
f_{\beta}(U)=-\frac{1}{\beta} \lim _{|\Lambda| \rightarrow \infty}|\Lambda|^{-1} \log \operatorname{Tr}\left\{e^{-\beta H_{\Lambda}}\right\},
$$

of specific ground state energy $e(U)=\lim _{\beta \rightarrow \infty} f_{\beta}(U)$, and of Schwinger functions, defined as follows.

Let us introduce the two component fermionic operators $\Psi_{\vec{x}, \sigma}^{ \pm}=\left(a_{\vec{x}, \sigma}^{ \pm}, b_{\vec{x}+\vec{\delta}_{1}, \sigma}^{ \pm}\right)$and let us write $\Psi_{\vec{x}, \sigma, 1}^{ \pm}=a_{\vec{x}, \sigma}^{ \pm}$and $\Psi_{\vec{x}, \sigma, 2}^{ \pm}=b_{\vec{x}+\vec{\delta}_{1}, \sigma}^{ \pm}$. We shall also consider the operators $\Psi_{\mathbf{x}, \sigma}^{ \pm}=$ $e^{H x_{0}} \Psi_{\vec{x}, \sigma}^{ \pm} e^{-H x_{0}}$ with $\mathbf{x}=\left(x_{0}, \vec{x}\right)$ and $x_{0} \in[0, \beta]$, for some $\beta>0$; we shall call $x_{0}$ the time variable. We shall write $\Psi_{\mathbf{x}, \sigma, 1}^{ \pm}=a_{\mathbf{x}, \sigma}^{ \pm}$and $\Psi_{\mathbf{x}, \sigma, 2}^{ \pm}=b_{\mathbf{x}+\boldsymbol{\delta}_{1}, \sigma}^{ \pm}$, with $\boldsymbol{\delta}_{1}=\left(0, \vec{\delta}_{1}\right)$. We define

$$
S_{n}^{\beta, \Lambda}\left(\mathbf{x}_{1}, \varepsilon_{1}, \sigma_{1}, \rho_{1} ; \ldots ; \mathbf{x}_{n}, \varepsilon_{n}, \sigma_{n}, \rho_{n}\right)=\left\langle\mathbf{T}\left\{\Psi_{\mathbf{x}_{1}, \sigma_{1}, \rho_{1}}^{\varepsilon_{1}} \cdots \Psi_{\mathbf{x}_{n}, \sigma_{n}, \rho_{n}}^{\varepsilon_{n}}\right\}\right\rangle_{\beta, \Lambda}
$$

where: $\mathbf{x}_{i} \in[0, \beta] \times \Lambda, \sigma_{i}=\uparrow \downarrow, \varepsilon_{i}= \pm, \rho_{i}=1,2$ and $\mathbf{T}$ is the operator of fermionic time ordering, acting on a product of fermionic fields as:

$$
\mathbf{T}\left(\Psi_{\mathbf{x}_{1}, \sigma_{1}, \rho_{1}}^{\varepsilon_{1}} \cdots \Psi_{\mathbf{x}_{n}, \sigma_{n}, \rho_{n}}^{\varepsilon_{n}}\right)=(-1)^{\pi} \Psi_{\mathbf{x}_{\pi(1)}, \sigma_{\pi(1)}, \rho_{\pi(1)}}^{\varepsilon_{\pi(1)}} \cdots \Psi_{\mathbf{x}_{\pi(n)}, \sigma_{\pi(n)}, \rho_{\pi(n)}}^{\varepsilon_{\pi(n)}}
$$

where $\pi$ is a permutation of $\{1, \ldots, n\}$, chosen in such a way that $x_{\pi(1) 0} \geq \cdots \geq x_{\pi(n) 0}$, and $(-1)^{\pi}$ is its sign. [If some of the time coordinates are equal each other, the arbitrariness of the definition is solved by ordering each set of operators with the same time coordinate so that creation operators precede the annihilation operators.]

Taking the limit $\Lambda \rightarrow \infty$ in (2.4) we get the finite temperature $n$-point Schwinger functions, denoted by $S_{n}^{\beta}\left(\mathbf{x}_{1}, \varepsilon_{1}, \sigma_{1}, \rho_{1} ; \ldots ; \mathbf{x}_{n}, \varepsilon_{n}, \sigma_{n}, \rho_{n}\right)$, which describe the properties of the infinite volume system at finite temperature. Taking the $\beta \rightarrow \infty$ limit of the finite temperature Schwinger functions, we get the zero temperature Schwinger functions, simply denoted by $S_{n}\left(\mathbf{x}_{1}, \varepsilon_{1}, \sigma_{1}, \rho_{1} ; \ldots ; \mathbf{x}_{n}, \varepsilon_{n}, \sigma_{n}, \rho_{n}\right)$, which describe the properties of the ground state of (2.1) in the thermodynamic limit (note that in this case, by the uniqueness of the ground state proved in [16], the infinite volume and zero temperature limits commute).

\section{B. The non interacting case}

In the non-interacting case $U=0$ the Schwinger functions of any order $n$ can be exactly computed as linear combinations of products of two-point Schwinger functions, via the wellknown Wick rule. The two-point Schwinger function itself, also called the free propagator, for $\mathbf{x} \neq \mathbf{y}$ and $\mathbf{x}-\mathbf{y} \neq( \pm \beta, \overrightarrow{0})$, is equal to (see Appendix $\mathrm{A}$ for details):

$$
\begin{aligned}
S_{0}^{\beta, \Lambda}(\mathbf{x}-\mathbf{y})_{\rho, \rho^{\prime}} & \left.\equiv S_{2}^{\beta, \Lambda}\left(\mathbf{x}, \sigma,-, \rho ; \mathbf{y}, \sigma,+, \rho^{\prime}\right)\right|_{U=0}= \\
& =\lim _{M \rightarrow \infty} \frac{1}{\beta|\Lambda|} \sum_{\mathbf{k} \in \mathcal{D}_{\beta, L}} \frac{e^{-i \mathbf{k} \cdot(\mathbf{x}-\mathbf{y})}}{k_{0}^{2}+|v(\vec{k})|^{2}}\left(\begin{array}{cc}
i k_{0} & -v^{*}(\vec{k}) \\
-v(\vec{k}) & i k_{0}
\end{array}\right)_{\rho, \rho^{\prime}}
\end{aligned}
$$

where:

1. $M \in \mathbb{N}, \mathbf{k}=\left(k_{0}, \vec{k}\right)$ and $\mathcal{D}_{\beta, L}=\mathcal{D}_{\beta} \times \mathcal{D}_{L} ;$

2. $\mathcal{D}_{\beta}=\left\{k_{0}=\frac{2 \pi}{\beta}\left(n_{0}+\frac{1}{2}\right): n_{0}=-M, \ldots, M-1\right\}$ and $\mathcal{D}_{L}=\left\{\vec{k}=\frac{n_{1}}{L} \vec{b}_{1}+\frac{n_{2}}{L} \vec{b}_{2}: 0 \leq\right.$ $\left.n_{1}, n_{2} \leq L-1\right\}$, where $\vec{b}_{1}=\frac{2 \pi}{3}(1, \sqrt{3}), \vec{b}_{2}=\frac{2 \pi}{3}(1,-\sqrt{3})$ are a basis of the dual lattice $\Lambda^{*}$;

3. $v(\vec{k})=\sum_{i=1}^{3} e^{i \vec{k}\left(\vec{\delta}_{i}-\vec{\delta}_{1}\right)}=1+2 e^{-i 3 / 2 k_{1}} \cos \frac{\sqrt{3}}{2} k_{2} ;$ its modulus $|v(\vec{k})|$ is the dispersion relation, given by

$$
\left|v_{\vec{k}}\right|=\sqrt{\left(1+2 \cos \left(3 k_{1} / 2\right) \cos \left(\sqrt{3} k_{2} / 2\right)\right)^{2}+4 \sin ^{2}\left(3 k_{1} / 2\right) \cos ^{2}\left(\sqrt{3} k_{2} / 2\right)} .
$$


At $\mathbf{x}=\mathbf{y}$ or $\mathbf{x}-\mathbf{y}=( \pm \beta, \overrightarrow{0})$, the free propagator has a jump discontinuity, see discussion at the end of Appendix $\mathrm{A}$. Note that $S_{0}^{\beta, \Lambda}(\mathbf{x})$ is antiperiodic in $x_{0}$, i.e. $S_{0}^{\beta, \Lambda}\left(x_{0}+\beta, \vec{x}\right)=$ $-S_{0}^{\beta, \Lambda}\left(x_{0}, \vec{x}\right)$, and that its Fourier transform $\hat{S}_{0}^{\beta, \Lambda}(\mathbf{k})$ is well-defined for any $\mathbf{k} \in \mathcal{D}_{\beta, L}$, even in the thermodynamic limit $L \rightarrow \infty$, since $\left|k_{0}\right| \geq \frac{\pi}{\beta}$. We shall refer to this last property by saying that the inverse temperature $\beta$ acts as an infrared cutoff for our theory.

If we take $\beta, L \rightarrow \infty$, the limiting propagator $\hat{S}_{0}(\mathbf{k})$ becomes singular at $\left\{k_{0}=0\right\} \times\left\{\vec{k}=\vec{p}_{F}^{ \pm}\right\}$, where

$$
\vec{p}_{F}^{ \pm}=\left(\frac{2 \pi}{3}, \pm \frac{2 \pi}{3 \sqrt{3}}\right)
$$

are the Fermi points (also called Dirac points, for an analogy with massive $\mathrm{QED}_{2+1}$ that will become clearer below). Note that the asymptotic behavior of $v(\vec{k})$ close to the Fermi points is given by $v\left(\vec{p}_{F}^{ \pm}+\vec{k}^{\prime}\right) \simeq \frac{3}{2}\left(i k_{1}^{\prime} \pm k_{2}^{\prime}\right)$. In particular, if $\omega= \pm$, the Fourier transform of the 2-point Schwinger function close to the Fermi point $\vec{p}_{F}^{\omega}$ can be rewritten in the form:

$$
\hat{S}_{0}\left(k_{0}, \vec{p}_{F}^{\omega}+\vec{k}^{\prime}\right)=\frac{1}{Z_{0}}\left(\begin{array}{cc}
-i k_{0} & -v_{F}^{(0)}\left(-i k_{1}^{\prime}+\omega k_{2}^{\prime}\right)+r_{\omega}\left(\vec{k}^{\prime}\right) \\
-v_{F}^{(0)}\left(i k_{1}^{\prime}+\omega k_{2}^{\prime}\right)+r_{\omega}^{*}\left(\vec{k}^{\prime}\right) & -i k_{0}
\end{array}\right)^{-1},
$$

where $Z_{0}=1$ is the free wave function renormalization and $v_{F}^{(0)}=3 / 2$ is the free Fermi velocity. Moreover, $\left|r_{\omega}\left(\vec{k}^{\prime}\right)\right| \leq C\left|\vec{k}^{\prime}\right|^{2}$, for small values of $\vec{k}^{\prime}$ and for some positive constant $C$.

\section{The interacting case}

We are now interested in what happens by adding a local interaction. In the case $U \neq 0$, the Schwinger functions are not exactly computable anymore. It is well-known that they can be written as formal power series in $U$, constructed in terms of Feynmann diagrams, using as free propagator the function $S_{0}(\mathbf{x})$ in (2.6). Our main result consists in a proof of convergence of this formal expansion for $U$ small enough, after the implementation of suitable resummations of the original power series. Our main result can be described as follows.

Theorem 1. Let us consider the 2D Hubbard model on the honeycomb lattice at half filling, defined by (2.1). There exist a constant $U_{0}>0$ such that, if $|U| \leq U_{0}$, the specific free energy $f_{\beta}(U)$ and the finite temperature Schwinger functions are analytic functions of $U$, uniformly in $\beta$ as $\beta \rightarrow \infty$, and so are the specific ground state energy $e(U)$ and the zero temperature Schwinger functions. The Fourier transform of the zero temperature two point Schwinger function $S(\mathbf{x})_{\rho, \rho^{\prime}} \stackrel{\text { def }}{=} S_{2}\left(\mathbf{x}, \sigma,-, \rho ; \mathbf{0}, \sigma,+, \rho^{\prime}\right)$, denoted by $\hat{S}(\mathbf{k})$, is singular only at the Fermi points $\mathbf{k}=\mathbf{p}_{F}^{ \pm}=\left(0, \vec{p}_{F}^{ \pm}\right)$, see (2.8), and, close to the singularities, if $\omega= \pm$, it can be written as

$$
\hat{S}\left(k_{0}, \vec{p}_{F}^{\omega}+\vec{k}^{\prime}\right)=\frac{1}{Z}\left(\begin{array}{cc}
-i k_{0} & -v_{F}\left(-i k_{1}^{\prime}+\omega k_{2}^{\prime}\right) \\
-v_{F}\left(i k_{1}^{\prime}+\omega k_{2}^{\prime}\right) & -i k_{0}
\end{array}\right)^{-1}\left(\mathbb{1}+R\left(\mathbf{k}^{\prime}\right)\right),
$$

with $\mathbf{k}^{\prime}=\left(k_{0}, \vec{k}^{\prime}\right)$, and with $Z$ and $v_{F}$ two real constants such that

$$
Z=1+a U^{2}+O\left(U^{3}\right), \quad v_{F}=\frac{3}{2}+b U^{2}+O\left(U^{3}\right)
$$

where $a$ and $b$ are non-vanishing constants. Moreover the matrix $R\left(\mathbf{k}^{\prime}\right)$ satisfies $\left\|R\left(\mathbf{k}^{\prime}\right)\right\| \leq C\left|\mathbf{k}^{\prime}\right|^{\vartheta}$ for some constants $C, \vartheta>0$ and for $\left|\mathbf{k}^{\prime}\right|$ small enough.

\section{Remarks.}

1) Theorem 1 says that the location of the singularity does not change in the presence of interaction; on the contrary, the wave function renormalization and Fermi velocity are modified by the interaction. Note also that, in the presence of the interaction, the Fermi velocity remains the same in the two coordinate direction even though the model does not display $90^{\circ}$ discrete rotational symmetry, but rather a $120^{\circ}$ rotational symmetry. 
2) The resulting theory is not quasi-free: the Wick rule is not valid anymore in the presence of interactions. However, the long distance asymptotics of the higher order Schwinger functions can be estimated by the same methods used to prove Theorem 1, and it is the same suggested by the Wick rule.

3) The fact that the interacting correlations decay as in the non-interacting case implies in particular the absence of long range order at zero temperature, e.g., the absence of Néel order in the ground state at weak coupling. In fact, as a corollary of our construction, we find:

$$
\left|\lim _{\beta,|\Lambda| \rightarrow \infty}\left\langle\vec{S}_{\vec{x}} \cdot \vec{S}_{\vec{y}}\right\rangle_{\beta, \Lambda}\right| \leq C \frac{1}{|\vec{x}-\vec{y}|^{4}},
$$

where, if $\vec{x} \in \Lambda$, the spin operator $\vec{S}_{\vec{x}}$ is defined as: $\vec{S}_{\vec{x}}=a_{\vec{x}, \cdot}^{+} \vec{\sigma} a_{\vec{x}, \cdot}^{-}$, with $\sigma_{i}, i=1,2,3$, the Pauli matrices; similarly, if $\vec{x} \in \Lambda+\vec{\delta}_{1}, \vec{S}_{\vec{x}}=\sum_{\sigma} b_{\vec{x}, \cdot}^{+} \vec{\sigma} b_{\vec{x}, \cdot}^{-}$. Note that it is well known that the ground state has zero total spin [16], however existence of Néel order was neither proven nor ruled out by the results in [16].

4) Similarly to what remarked in the previous item, one can exclude the existence of superconducting long range order: the Cooper pairs correlations decay to zero at infinity at least as fast as the spin-spin correlations in (2.12).

5) Our analysis can be extended in a straightforward way to the case of exponentially decaying interactions (instead of local interactions). However, if the decay is slower, the result may change. In particular, in the presence of 3D Coulomb interactions, the electron-electron interaction becomes marginal (instead of irrelevant), in a renormalization group sense [12].

6) Previous analyses of the Hubbard model on the honeycomb lattice were performed only at a perturbative level, without any control on the convergence of the weak coupling expansion, and directly in the Quantum Field Theory approximation, consisting in the replacement of $\hat{S}_{0}(\mathbf{k})$ by its linear approximation around the Fermi points, see for instance [1] and references therein.

7) In Appendix $\mathrm{C}$ we prove that indeed the 2D Hubbard model on a honeycomb lattice is asymptotically described by a $\mathrm{QED}_{2+1}$ model in the presence of an ultraviolet cutoff, massive "photons" and massless electrons, provided that the bare parameters of the $\mathrm{QED}_{2+1}$ are carefully chosen to include lattice effects. As a result, the correlations asymptotically verify a modified Ward Identity (WI) related to an approximate local $U(1)$ Lorentz symmetry: note, however, that the renormalized charge velocity appearing in the modified WI for graphene explicitly breaks rotational invariance, contrary to what happens to the Fermi velocity, or to the charge velocity of a pure relativistic QED model.

The proof of the Theorem is based on constructive fermionic Renormalization Group (RG) methods, see [2, 18, 22] for extensive reviews. It is worth remarking that the result summarized in Theorem 1 is one of the few rigorous construction of the ground state properties (including correlations) of a weak coupling 2D Hubbard model. The only other example we are aware of is the Fermi liquid construction in [8], applicable to cases of weakly interacting 2D Fermi systems with a highly asymmetric interacting Fermi surface. Related results include the construction of the state at temperatures larger than a BCS-like critical temperature [3, 7], or the computation of the first contribution to the ground state energy in a weak coupling limit [10, 17, 23].

The rest of the paper will be devoted to the proof of Theorem 1. In Sec. III A we review the Grassmann integral representation for the free energy and the Schwinger functions. In Sec IIIB we start to describe the integration procedure leading to the computation of the free energy, and in particular we describe how to integrate out the ultraviolet degrees of freedom. In Sec IIIC we complete the proof of convergence of the series for the free energy and the ground state energy. In Sec IIID we describe the proof of convergence for the series for the Schwinger functions, with particular emphasis to the case of the two-point Schwinger function. In the Appendices we provide further details concerning the non-interacting theory, the ultraviolet integration and the equivalence (as far as the long distance behavior is concerned) between the Hubbard model and a massive QED theory in $2+1$ dimensions. 


\section{RENORMALIZATION GROUP ANALYSIS}

\section{A. Grassmann Integration}

It is well-known that the usual formal power series in $U$ for the partition function and for the Schwinger functions of model (2.1) can be equivalently rewritten in terms of Grassmann functional integrals, defined as follows.

We consider the Grassmann algebra generated by the Grassmannian variables $\left\{\hat{\Psi}_{\mathbf{k}, \sigma, \rho}^{ \pm}\right\}_{\mathbf{k} \in \mathcal{D}_{\beta, L}=\uparrow \downarrow, \rho=1,2}^{\sigma}$ and a Grassmann integration $\int\left[\prod_{\mathbf{k} \in \mathcal{D}_{\beta, L}} \prod_{\sigma=\uparrow \downarrow}^{\rho=1,2} d \hat{\Psi}_{\mathbf{k}, \sigma, \rho}^{+} d \hat{\Psi}_{\mathbf{k}, \sigma, \rho}^{-}\right]$defined as the linear operator on the Grassmann algebra such that, given a monomial $Q\left(\hat{\Psi}^{-}, \hat{\Psi}^{+}\right)$ in the variables $\hat{\Psi}_{\mathbf{k}, \sigma, \rho}^{ \pm}$, its action on $Q\left(\hat{\Psi}^{-}, \hat{\Psi}^{+}\right)$is 0 except in the case $Q\left(\hat{\Psi}^{-}, \hat{\Psi}^{+}\right)=$ $\prod_{\mathbf{k} \in \mathcal{D}_{\beta, L}} \prod_{\sigma=\uparrow \downarrow}^{\rho=1,2} \hat{\Psi}_{\mathbf{k}, \sigma, \rho}^{-} \hat{\Psi}_{\mathbf{k}, \sigma, \rho}^{+}$, up to a permutation of the variables. In this case the value of the integral is determined, by using the anticommuting properties of the variables, by the condition

$$
\int\left[\prod_{\mathbf{k} \in \mathcal{D}_{\beta}, L} \prod_{\sigma=\uparrow \downarrow}^{\rho=1,2} d \hat{\Psi}_{\mathbf{k}, \sigma, \rho}^{+} d \hat{\Psi}_{\mathbf{k}, \sigma, \rho}^{-}\right] \prod_{\mathbf{k} \in \mathcal{D}_{\beta, L}} \prod_{\sigma=\uparrow \downarrow}^{\rho=1,2} \hat{\Psi}_{\mathbf{k}, \sigma, \rho}^{-} \hat{\Psi}_{\mathbf{k}, \sigma, \rho}^{+}=1
$$

Defining the free propagator matrix $\hat{g}_{\mathbf{k}}$ as

$$
\hat{g}_{\mathbf{k}}=\left(\begin{array}{cc}
-i k_{0} & -v^{*}(\vec{k}) \\
-v(\vec{k}) & -i k_{0}
\end{array}\right)^{-1}
$$

and the "Gaussian integration" $P(d \Psi)$ as

$$
\begin{array}{r}
P(d \Psi)=\left[\prod_{\mathbf{k} \in \mathcal{D}_{\beta, L}}^{\sigma=\uparrow \downarrow} \frac{-\beta^{2}|\Lambda|^{2}}{k_{0}^{2}+|v(\vec{k})|^{2}} d \hat{\Psi}_{\mathbf{k}, \sigma, 1}^{+} d \hat{\Psi}_{\mathbf{k}, \sigma, 1}^{-} d \hat{\Psi}_{\mathbf{k}, \sigma, 2}^{+} d \hat{\Psi}_{\mathbf{k}, \sigma, 2}^{-}\right] \\
\cdot \exp \left\{-(\beta|\Lambda|)^{-1} \sum_{\mathbf{k} \in \mathcal{D}_{\beta, L}}^{\sigma=\uparrow \downarrow} \hat{\Psi}_{\mathbf{k}, \sigma, \cdot}^{+} \hat{g}_{\mathbf{k}}^{-1} \hat{\Psi}_{\mathbf{k}, \sigma, \cdot}^{-}\right\}
\end{array}
$$

it turns out that

$$
\int P(d \Psi) \hat{\Psi}_{\mathbf{k}_{1}, \sigma_{1}, \rho_{1}}^{-} \hat{\Psi}_{\mathbf{k}_{2}, \sigma_{2}, \rho_{2}}^{+}=\beta|\Lambda| \delta_{\sigma_{1}, \sigma_{2}} \delta_{\mathbf{k}_{1}, \mathbf{k}_{2}}\left[\hat{g}_{\mathbf{k}_{1}}\right]_{\rho_{1}, \rho_{2}},
$$

so that, if $\mathbf{x}-\mathbf{y} \notin \beta \mathbb{Z} \times\{\overrightarrow{0}\}$,

$$
\lim _{M \rightarrow \infty} \frac{1}{\beta|\Lambda|} \sum_{\mathbf{k} \in \mathcal{D}_{\beta, L}} e^{-i \mathbf{k}(\mathbf{x}-\mathbf{y})} \hat{g}_{\mathbf{k}}=\lim _{M \rightarrow \infty} \int P(d \Psi) \Psi_{\mathbf{x}, \sigma}^{-} \Psi_{\mathbf{y}, \sigma}^{+}=S_{0}(\mathbf{x}-\mathbf{y})
$$

where $S_{0}(\mathbf{x}-\mathbf{y})$ was defined in (2.5) and the Grassmann fields $\Psi_{\mathbf{x}, \sigma}^{ \pm}$are defined by

$$
\Psi_{\mathbf{x}, \sigma, \rho}^{ \pm}=\frac{1}{\beta|\Lambda|} \sum_{\mathbf{k} \in \mathcal{D}_{\beta, L}} e^{ \pm i \mathbf{k x}} \hat{\Psi}_{\mathbf{k}, \sigma, \rho}^{ \pm}, \quad \mathbf{x} \in \Lambda_{\beta, M} \times \Lambda
$$

with $\Lambda_{\beta, M}=\{m \beta / M: m=-M, \ldots, M-1\}$. Let us now consider the function on the Grassmann algebra

$$
\begin{aligned}
V(\Psi) & =U \sum_{\rho=1,2} \int d \mathbf{x} \Psi_{\mathbf{x}, \uparrow, \rho}^{+} \Psi_{\mathbf{x}, \uparrow, \rho}^{-} \Psi_{\mathbf{x}, \downarrow, \rho}^{+} \Psi_{\mathbf{x}, \downarrow, \rho}^{-}= \\
& =\frac{U}{(\beta|\Lambda|)^{3}} \sum_{\rho=1,2} \sum_{\mathbf{k}, \mathbf{k}^{\prime}, \mathbf{p}} \hat{\Psi}_{\mathbf{k}-\mathbf{p}, \uparrow, \rho}^{+} \hat{\Psi}_{\mathbf{k}, \uparrow, \rho}^{-} \hat{\Psi}_{\mathbf{k}^{\prime}+\mathbf{p}, \downarrow, \rho}^{+} \hat{\Psi}_{\mathbf{k}^{\prime}, \downarrow, \rho}^{-}
\end{aligned}
$$


where, in the first line, the symbol $\int d \mathbf{x}$ must be interpreted as

$$
\int d \mathbf{x}=\frac{\beta}{2 M} \sum_{x_{0} \in \Lambda_{\beta, M}} \sum_{\vec{x} \in \Lambda}
$$

and, in the second line, the sums over $\mathbf{k}, \mathbf{k}^{\prime}$ run over the set $\mathcal{D}_{\beta, L}$, while the sums over $\mathbf{p}$ run over the set $2 \pi \beta^{-1} \mathbb{Z} \times \mathcal{D}_{L}$ (p is the transferred momentum). Note that the integral $\int P(d \Psi) e^{-V(\Psi)}$ is well defined for any $U$; it is indeed a polynomial in $U$, of degree depending on $M$ and $L$. Standard arguments show that, if there exists the limit of $\int P(d \Psi) e^{-V(\Psi)}$ as $M \rightarrow \infty$, then the normalized partition function can be written as

$$
e^{-\beta|\Lambda| F_{\beta, L}} \stackrel{\text { def }}{=} \frac{\operatorname{Tr}\left[e^{-\beta H_{\Lambda}}\right]}{\operatorname{Tr}\left[e^{-\beta H_{0}}\right]}=\lim _{M \rightarrow \infty} \int P(d \Psi) e^{-V(\Psi)}
$$

where $H_{0}$ is equal to (2.1) with $U=0$. A possible way to prove (3.21) is to compare the perturbation theory obtained by expanding in powers of $U$ via Trotter's product formula the trace $\operatorname{Tr}\left\{e^{-\beta H_{\Lambda}}\right\} / \operatorname{Tr}\left\{e^{-\beta H_{0}}\right\}$ with the one obtained by expanding in $U$ the Grassmann functional integral, and then show that they are the same, order by order, see [1]. This proof also shows that the correct choice of the interaction (3.19) expressed in Grassmann variables does not include terms bilinear in the fields, contrary to the interaction in second quantized form, see (2.1): in fact, with this choice, in both perturbative expansions the "tadpoles" are exactly vanishing, as required by the condition that the system is at half filling.

Similarly, the Schwinger functions at distinct space-time points, defined in (2.4), can be computed as

$$
S\left(\mathbf{x}_{1}, \sigma_{1}, \varepsilon_{1}, \rho_{1} ; \ldots ; \mathbf{x}_{n}, \sigma_{n}, \varepsilon_{n}, \rho_{n}\right)=\lim _{M \rightarrow \infty} \frac{\int P(d \Psi) e^{-V(\Psi)} \Psi_{\mathbf{x}_{1}, \sigma_{1}, \rho_{1}}^{\varepsilon_{1}} \cdots \Psi_{\mathbf{x}_{n}, \sigma_{n}, \rho_{n}}^{\varepsilon_{n}}}{\int P(d \Psi) e^{-V(\Psi)}} .
$$

Note that the limit $\mathbf{x}_{i}-\mathbf{x}_{j} \rightarrow \mathbf{0}$ and the limit $M \rightarrow \infty$ do not commute in general.

In the following we shall study the functional integrals by introducing suitable expansions where the value of $M$ plays no essential role and we shall indeed be able to control such expansions uniformly in $M$, if $U$ is small enough and that the limit $M \rightarrow \infty$ can be taken in the resulting expressions for the free energy and the Schwinger functions. For this reason, from now on we shall not stress anymore the dependence on $M$, unless for the cases where the presence of a finite $M$ is relevant, e.g., for the analysis of the ultraviolet integration described in Appendix A.

It is important to note that both the Gaussian integration $P(d \Psi)$ and the interaction $V(\Psi)$ are invariant under the action of a number of remarkable symmetry transformations, which will be preserved by the subsequent iterative integration procedure and will guarantee the vanishing of some running coupling constants (see below for details). Let us collect in the following lemma all the symmetry properties we will need in the following.

Lemma 1. For any choice of $M, \beta, \Lambda$, both the quadratic Grassmann measure $P(d \Psi)$ defined in (3.15) and the quartic Grassmann interaction $V(\Psi)$ defined in (3.19) are invariant under the following transformations:

(1) spin exchange: $\hat{\Psi}_{\mathbf{k}, \sigma, \rho}^{\varepsilon} \longleftrightarrow \hat{\Psi}_{\mathbf{k},-\sigma, \rho}^{\varepsilon}$;

(2) global $U(1): \hat{\Psi}_{\mathbf{k}, \sigma, \rho}^{\varepsilon} \rightarrow e^{i \varepsilon \alpha_{\sigma}} \hat{\Psi}_{\mathbf{k}, \sigma, \rho}^{\varepsilon}$, with $\alpha_{\sigma} \in \mathbb{R}$ independent of $\mathbf{k}$;

(3) $\operatorname{spin} S O(2):\left(\begin{array}{c}\hat{\Psi}_{\mathbf{k}, \uparrow, \rho}^{\varepsilon} \\ \hat{\Psi}_{\mathbf{k}, \downarrow, \rho}^{\varepsilon}\end{array}\right) \rightarrow R_{\theta}\left(\begin{array}{c}\hat{\Psi}_{\mathbf{k}, \uparrow, \rho}^{\varepsilon} \\ \hat{\Psi}_{\mathbf{k}, \downarrow, \rho}^{\varepsilon}\end{array}\right)$, with $R_{\theta}=\left(\begin{array}{cc}\cos \theta & \sin \theta \\ -\sin \theta & \cos \theta\end{array}\right)$ and $\theta \in \mathbb{T}$ independent of $\mathbf{k}$;

(4) discrete spatial rotations: $\hat{\Psi}_{\left(k_{0}, \vec{k}\right), \sigma, \rho}^{ \pm} \rightarrow e^{\mp i \vec{k}\left(\vec{\delta}_{3}-\vec{\delta}_{1}\right)(\rho-1)} \hat{\Psi}_{\left(k_{0}, T_{1} \vec{k}\right), \sigma, \rho}^{ \pm}$, with $T_{1} \vec{x} \stackrel{\text { def }}{=} R_{2 \pi / 3} \vec{x}$; note that in real space this transformation simply reads $a_{\left(x_{0}, \vec{x}\right), \sigma}^{ \pm} \rightarrow a_{\left(x_{0}, T_{1} \vec{x}\right), \sigma}^{ \pm}$and $b_{\left(x_{0}, \vec{x}\right), \sigma}^{ \pm} \rightarrow b_{\left(x_{0}, T_{1} \vec{x}\right), \sigma}^{ \pm}$

(5) complex conjugation: $\hat{\Psi}_{\mathbf{k}, \sigma, \rho}^{ \pm} \rightarrow \hat{\Psi}_{-\mathbf{k}, \sigma, \rho}^{ \pm}, c \rightarrow c^{*}$, where $c$ is a generic constant appearing in $P(d \Psi)$ and/or in $V(\Psi)$;

(6.a) horizontal reflections: $\hat{\Psi}_{\left(k_{0}, k_{1}, k_{2}\right), \sigma, 1}^{ \pm} \longleftrightarrow \hat{\Psi}_{\left(k_{0},-k_{1}, k_{2}\right), \sigma, 2}^{ \pm}$; 
(6.b) vertical reflections: $\hat{\Psi}_{\left(k_{0}, k_{1}, k_{2}\right), \sigma, \rho}^{ \pm} \rightarrow \hat{\Psi}_{\left(k_{0}, k_{1},-k_{2}\right), \sigma, \rho}^{ \pm}$;

(7) particle-hole: $\hat{\Psi}_{\left(k_{0}, \vec{k}\right), \sigma, \rho}^{ \pm} \rightarrow i \hat{\Psi}_{\left(k_{0},-\vec{k}\right), \sigma, \rho}^{\mp}$.

(8) $\underline{\text { inversion: }} \hat{\Psi}_{\left(k_{0}, \vec{k}\right), \sigma, \rho}^{ \pm} \rightarrow i(-1)^{\rho} \hat{\Psi}_{\left(-k_{0}, \vec{k}\right), \sigma, \rho}^{ \pm}$.

Proof. A moment's thought shows that the invariance of $V(\Psi)$ under the above symmetries is obvious, and so is the invariance of $P(d \Psi)$ under (1)-(2)-(3). Let us then prove the invariance of $P(d \Psi)$ under (4)-(5)-(6.a)-(6.b)-(7)-(8). More precisely, let us consider the term

$$
\begin{aligned}
& \sum_{\mathbf{k}} \hat{\Psi}_{\mathbf{k}, \sigma,}^{+} \hat{g}_{\mathbf{k}}^{-1} \hat{\Psi}_{\mathbf{k}, \sigma, \cdot}^{-}= \\
& -i \sum_{\mathbf{k}} \hat{\Psi}_{\mathbf{k}, \sigma, 1}^{+} k_{0} \hat{\Psi}_{\mathbf{k}, \sigma, 1}^{-}-\sum_{\mathbf{k}} \hat{\Psi}_{\mathbf{k}, \sigma, 1}^{+} v^{*}(\vec{k}) \hat{\Psi}_{\mathbf{k}, \sigma, 2}^{-}-\sum_{\mathbf{k}} \hat{\Psi}_{\mathbf{k}, \sigma, 2}^{+} v(\vec{k}) \hat{\Psi}_{\mathbf{k}, \sigma, 1}^{-}-i \sum_{\mathbf{k}} \hat{\Psi}_{\mathbf{k}, \sigma, 2}^{+} k_{0} \hat{\Psi}_{\mathbf{k}, \sigma, 2}^{-}
\end{aligned}
$$

in (3.15), and let us prove its invariance under the transformations (4)-(5)-(6.a)-(6.b)-(7)-(8).

Under the transformation (4), the first and fourth term in the second line of (3.23) are obviously invariant, while the sum of the second and third is changed into

$$
\begin{aligned}
& -\sum_{\mathbf{k}}\left[\hat{\Psi}_{\left(k_{0}, T_{1} \vec{k}\right), \sigma, 1}^{+} v^{*}(\vec{k}) e^{+i \vec{k}\left(\vec{\delta}_{3}-\vec{\delta}_{1}\right)} \hat{\Psi}_{\left(k_{0}, T_{1} \vec{k}\right), \sigma, 2}^{-}+\hat{\Psi}_{\left(k_{0}, T_{1} \vec{k}\right), \sigma, 2}^{+} e^{-i \vec{k}\left(\vec{\delta}_{3}-\vec{\delta}_{1}\right)} v(\vec{k}) \hat{\Psi}_{\left(k_{0}, T_{1} \vec{k}\right), \sigma, 1}^{-}\right]= \\
& =-\sum_{\mathbf{k}}\left[\hat{\Psi}_{\mathbf{k}, \sigma, 1}^{+} v^{*}\left(T_{1}^{-1} \vec{k}\right) e^{+i \vec{k}\left(\vec{\delta}_{1}-\vec{\delta}_{2}\right)} \hat{\Psi}_{\mathbf{k}, \sigma, 2}^{-}+\hat{\Psi}_{\mathbf{k}, \sigma, 2}^{+} e^{-i \vec{k}\left(\vec{\delta}_{1}-\vec{\delta}_{2}\right)} v\left(T_{1}^{-1} \vec{k}\right) \hat{\Psi}_{\mathbf{k}, \sigma, 1}^{-}\right] .
\end{aligned}
$$

Using that $v\left(T_{1}^{-1} \vec{k}\right)=e^{i \vec{k}\left(\vec{\delta}_{1}-\vec{\delta}_{2}\right)} v(\vec{k})$, as it follows by the definition $v(\vec{k})=\sum_{i=1,2,3} e^{i \vec{k}\left(\vec{\delta}_{i}-\vec{\delta}_{1}\right)}$, we find that the last line of (3.24) is equal to the sum of the second and third term in (3.23), as desired.

The invariance of (3.23) under the transformation (5) is very simple, if one notes that $v(-\vec{k})=$ $v^{*}(\vec{k})$, as it follows by the definition of $v(\vec{k})$.

Under the transformation (6.a), the sum of the first and fourth term in the second line of (3.23) is obviously invariant, while the sum of the second and third is changed into

$$
\begin{aligned}
& -\sum_{\mathbf{k}} \hat{\Psi}_{\left(k_{0},-k_{1}, k_{2}\right), \sigma, 2}^{+} v^{*}(\vec{k}) \hat{\Psi}_{\left(k_{0},-k_{1}, k_{2}\right), \sigma, 1}^{-}-\sum_{\mathbf{k}} \hat{\Psi}_{\left(k_{0},-k_{1}, k_{2}\right), \sigma, 1}^{+} v(\vec{k}) \hat{\Psi}_{\left(k_{0},-k_{1}, k_{2}\right), \sigma, 2}^{-}= \\
& =-\sum_{\mathbf{k}} \hat{\Psi}_{\mathbf{k}, \sigma, 2}^{+} v^{*}\left(\left(-k_{1}, k_{2}\right)\right) \hat{\Psi}_{\mathbf{k}, \sigma, 1}^{-}-\sum_{\mathbf{k}} \hat{\Psi}_{\mathbf{k}, \sigma, 1}^{+} v\left(\left(-k_{1}, k_{2}\right)\right) \hat{\Psi}_{\mathbf{k}, \sigma, 2}^{-} .
\end{aligned}
$$

Noting that $v\left(\left(-k_{1}, k_{2}\right)\right)=v^{*}(\mathbf{k})$, one sees that this is the same as the sum of the second and third term in (3.23), as desired.

Similarly, noting that $v\left(\left(k_{1},-k_{2}\right)\right)=v(\mathbf{k})$, one finds that (3.23) is invariant under the transformation (6.b).

Under the transformation (7), the sum of the first and fourth term in (3.23) is obviously invariant, while the sum of the second and third term is changed into

$$
\begin{aligned}
& +\sum_{\mathbf{k}} \hat{\Psi}_{\left(k_{0},-\vec{k}\right), \sigma, 1}^{-} v^{*}(\vec{k}) \hat{\Psi}_{\left(k_{0},-\vec{k}\right), \sigma, 2}^{+}+\sum_{\mathbf{k}} \hat{\Psi}_{\left(k_{0},-\vec{k}\right), \sigma, 2}^{-} v(\vec{k}) \hat{\Psi}_{\left(k_{0},-\vec{k}\right), \sigma, 1}^{+}= \\
& =-\sum_{\mathbf{k}} \hat{\Psi}_{\mathbf{k}, \sigma, 2}^{+} v^{*}(-\vec{k}) \hat{\Psi}_{\mathbf{k}, \sigma, 1}^{-}-\sum_{\mathbf{k}} \hat{\Psi}_{\mathbf{k}, \sigma, 1}^{+} v(-\vec{k}) \hat{\Psi}_{\mathbf{k}, \sigma, 2}^{-} .
\end{aligned}
$$

Using, again, that $v(-\vec{k})=v^{*}(\vec{k})$, we see that the latter sum is the same as the sum of the second and third term in (3.23), as desired.

Finally, under the transformation (8), all the terms in the right hand side of (3.23) are separately invariant, and the proof of Lemma 1 is concluded.

\section{B. Free energy: The ultraviolet integration}

We start by studying the partition function

$$
\Xi_{\beta, L}=e^{-\beta|\Lambda| F_{\beta, L}}=\int P(d \Psi) e^{-V(\Psi)} .
$$


Note that our lattice model has an intrinsic ultraviolet cut-off in the $\vec{k}$ variables, while the $k_{0}$ variable is unbounded. A preliminary step to our infrared analysis is the integration of the ultraviolet degrees of freedom corresponding to the large values of $k_{0}$. We proceed in the following way. We decompose the free propagator $\hat{g}_{\mathbf{k}}$ into a sum of two propagators supported in the regions of $k_{0}$ "large" and "small", respectively. The regions of $k_{0}$ large and small are defined in terms of a smooth support function $\chi_{0}(t)$ which is 1 for $t \leq a_{0}$ and 0 for $t \geq a_{0} \gamma$, $\gamma>1 ; a_{0}$ is chosen so that the support of $\chi_{0}\left(\sqrt{k_{0}^{2}+\left|\vec{k}-\vec{p}_{F}^{+}\right|^{2}}\right)$ and $\chi_{0}\left(\sqrt{k_{0}^{2}+\left|\vec{k}-\vec{p}_{F}\right|^{2}}\right)$ are disjoint (here $|\cdot|$ is the euclidean norm over $\mathbb{R}^{2} / \Lambda^{*}$ ). In order for this condition to be satisfied, it is enough that $2 a_{0} \gamma<4 \pi /(3 \sqrt{3})$; in the following, for reasons that will become clearer later, we shall assume the slightly more restrictive condition $2 a_{0} \gamma<4 \pi / 3-4 \pi /(3 \sqrt{3})$. We define

$$
f_{u . v .}(\mathbf{k})=1-\chi_{0}\left(\sqrt{k_{0}^{2}+\left|\vec{k}-\vec{p}_{F}^{+}\right|^{2}}\right)-\chi_{0}\left(\sqrt{k_{0}^{2}+\left|\vec{k}-\vec{p}_{F}\right|^{2}}\right)
$$

and $f_{i . r .}(\mathbf{k})=1-f_{u . v .}(\mathbf{k})$, so that we can rewrite $\hat{g}_{\mathbf{k}}$ as:

$$
\hat{g}_{\mathbf{k}}=f_{u . v .}(\mathbf{k}) \hat{g}_{\mathbf{k}}+f_{i . r .}(\mathbf{k}) \hat{g}_{\mathbf{k}} \stackrel{\text { def }}{=} \hat{g}^{(u . v .)}(\mathbf{k})+\hat{g}^{(i . r .)}(\mathbf{k}) .
$$

We now introduce two independent set of Grassmann fields $\left\{\Psi_{\mathbf{k}, \sigma, \rho}^{(u . v) \pm}\right\}$ and $\left\{\Psi_{\mathbf{k}, \sigma, \rho}^{(i . r .) \pm}\right\}$, with $\mathbf{k} \in \mathcal{D}_{\beta, L}, \sigma=\uparrow \downarrow, \rho=1,2$, and the Gaussian integrations $P\left(d \Psi^{(u . v .)}\right)$ and $P\left(d \Psi^{(i . r .)}\right)$ defined by

$$
\begin{gathered}
\int P\left(d \Psi^{(u . v .)}\right) \hat{\Psi}_{\mathbf{k}_{1}, \sigma_{1}, \rho_{1}}^{(u .)} \hat{\Psi}_{\mathbf{k}_{2}, \sigma_{2}, \rho_{2}}^{(u . v .)+}=\beta|\Lambda| \delta_{\sigma_{1}, \sigma_{2}} \delta_{\mathbf{k}_{1}, \mathbf{k}_{2}} \hat{g}^{(u . v .)}\left(\mathbf{k}_{1}\right)_{\rho_{1}, \rho_{2}}, \\
\int P\left(d \Psi^{(i . r .)}\right) \hat{\Psi}_{\mathbf{k}_{1}, \sigma_{1}, \rho_{1}}^{(u . v .)-} \hat{\Psi}_{\mathbf{k}_{2}, \sigma_{2}, \rho_{2}}^{(i . r .)+}=\beta|\Lambda| \delta_{\sigma_{1}, \sigma_{2}} \delta_{\mathbf{k}_{1}, \mathbf{k}_{2}} \hat{g}^{(i . r .)}\left(\mathbf{k}_{1}\right)_{\rho_{1}, \rho_{2}} .
\end{gathered}
$$

Similarly to $P(d \Psi)$, the Gaussian integrations $P\left(d \Psi^{(u \cdot v .)}\right), P\left(d \Psi^{(i . r .)}\right)$ also admit an explicit representation analogous to (3.14), with $\hat{g}_{\mathbf{k}}$ replaced by $\hat{g}^{(u \cdot v .)}(\mathbf{k})$ or $\hat{g}^{(i . r .)}(\mathbf{k})$ and the sum over $\mathbf{k}$ restricted to the values in the support of $f_{u . v .}(\mathbf{k})$ or $f_{i . r .}(\mathbf{k})$, respectively. It easy to verify that the ultraviolet propagator $g^{(u . v .)}(\mathbf{x}-\mathbf{y})=(\beta|\Lambda|)^{-1} \sum_{\mathbf{k} \in \mathcal{D}_{\beta, L}} e^{-i \mathbf{k}(\mathbf{x}-\mathbf{y})} \hat{g}^{(u \cdot v .)}(\mathbf{k})$ satisfies

$$
\left|g^{(u . v .)}(\mathbf{x}-\mathbf{y})\right| \leq \frac{C_{N}}{1+|\mathbf{x}-\mathbf{y}|^{N}} .
$$

The definition of Grassmann integration implies the following identity ("addition principle"):

$$
\int P(d \Psi) e^{-V(\Psi)}=\int P\left(d \Psi^{(i . r .)}\right) \int P\left(d \Psi^{(u . v .)}\right) e^{-V\left(\Psi^{(i . r .)}+\Psi^{(u . v .)}\right)}
$$

so that we can rewrite the partition function as

$$
\begin{aligned}
\Xi_{\beta, L} & =e^{-\beta|\Lambda| F_{L, \beta}}=\int P\left(d \Psi^{(i . r .)}\right) \exp \left\{\sum_{n \geq 1} \frac{1}{n !} \mathcal{E}_{u . v .}^{T}\left(-V\left(\Psi^{(i . r .)}+\cdot\right) ; n\right)\right\} \equiv \\
& \equiv e^{-\beta|\Lambda| F_{0}} \int P\left(d \Psi^{(i . r .)}\right) e^{-\mathcal{V}\left(\Psi^{(i . r .)}\right)},
\end{aligned}
$$

where the truncated expectation $\mathcal{E}_{u . v}^{T}$. is defined, given any polynomial $V_{1}\left(\Psi^{(u . v .)}\right)$ with coefficients depending on $\Psi^{(i . r .)}$, as

$$
\mathcal{E}_{u . v .}^{T}\left(V_{1}(\cdot) ; n\right)=\left.\frac{\partial^{n}}{\partial \lambda^{n}} \log \int P\left(d \Psi^{(u . v .)}\right) e^{\lambda V_{1}\left(\Psi^{(u . v .)}\right)}\right|_{\lambda=0}
$$

and $\mathcal{V}$ is fixed by the condition $\mathcal{V}(0)=0$. It can be shown (see discussion after (3.37) and Appendix $\mathrm{B}$ ) that $\mathcal{V}$ can be written as

$$
\begin{gathered}
\mathcal{V}(\Psi)=\sum_{n=1}^{\infty}(\beta|\Lambda|)^{-2 n} \sum_{\sigma_{1}, \ldots, \sigma_{n}=\uparrow \downarrow} \sum_{\rho_{1}, \ldots, \rho_{2 n}=1,2} \sum_{\mathbf{k}_{1}, \ldots, \mathbf{k}_{2 n}}\left[\prod_{j=1}^{n} \hat{\Psi}_{\mathbf{k}_{2 j-1}, \sigma_{j}, \rho_{2 j-1}}^{(i . r .)+} \hat{\Psi}_{\mathbf{k}_{2 j}, \sigma_{j}, \rho_{2 j}}^{(i . r)-}\right] . \\
\cdot \hat{W}_{2 n, \underline{\rho}}\left(\mathbf{k}_{1}, \ldots, \mathbf{k}_{2 n-1}\right) \delta\left(\sum_{j=1}^{n}\left(\mathbf{k}_{2 j-1}-\mathbf{k}_{2 j}\right)\right)
\end{gathered}
$$


where $\underline{\rho}=\left(\rho_{1}, \ldots, \rho_{2 n}\right)$ and we used the notation

$$
\delta(\mathbf{k})=\delta(\vec{k}) \delta\left(k_{0}\right), \quad \delta(\vec{k})=|\Lambda| \sum_{n_{1}, n_{2} \in \mathbb{Z}} \delta_{\vec{k}, n_{1} \vec{b}_{1}+n_{2} \vec{b}_{2}}, \quad \delta\left(k_{0}\right)=\beta \delta_{k_{0}, 0},
$$

with $\vec{b}_{1}, \vec{b}_{2}$ a basis of $\Lambda^{*}$. The possibility of representing $\mathcal{V}$ in the form (3.35), with the kernels $\hat{W}_{2 n, \underline{\rho}}$ independent of the spin indices $\sigma_{i}$, follows from the symmetries listed in Lemma 1 and from the remark that $P\left(d \Psi^{(u . v .)}\right)$ and $P\left(d \Psi^{(i . r .)}\right)$ are separately invariant under the same symmetries.

The constant $F_{0}$ in (3.33) and the kernels $\hat{W}_{2 n, \rho}$ in (3.35) are given by power series in $U$, convergent under the condition $|U| \leq U_{0}$, for $U_{0}$ small enough; after Fourier transform, the x-space counterparts of the kernels $\hat{W}_{2 n, \underline{\rho}}$ satisfy the following bounds:

$$
\int d \mathbf{x}_{1} \cdots d \mathbf{x}_{2 n}\left[\prod_{1 \leq i<j \leq 2 n}\left|\mathbf{x}_{i}-\mathbf{x}_{j}\right|^{m_{i, j}}\right]\left|W_{2 n, \underline{\rho}}\left(\mathbf{x}_{1}, \ldots, \mathbf{x}_{2 n}\right)\right| \leq \beta|\Lambda| C_{m}^{n}|U|^{\max \{1, n-1\}}
$$

for some constant $C_{m}>0$, where $m=\sum_{1<i<j<2 n} m_{i, j}$.

The proof of convergence of the power series defining $F_{0}$ and $W_{2 n, \rho}$, as well as the proof of the bounds (3.37), uses the decay property (3.31) combined with standard fermionic cluster expansion methods. The proof is much simpler than the infrared integration that we shall study below, and is based on similar ideas; see Appendix B for a proof. Note that the decay (3.31) suggests the possibility of a single scale integration of the ultraviolet degrees of freedom. However, the discontinuity of the propagator at $x_{0}-y_{0}=0$ implies that $\hat{g}_{\mathbf{k}}$ does not admit a Gram representation [21] and this fact prevents the direct implementation of a single step fermionic cluster expansion (see next section for the notion of Gram determinant and for a description of the use of Gram determinants in the infrared multiscale integration). A possible way out of this problem is to decompose the ultraviolet propagator as a sum of propagators, each admitting a Gram representation, and to perform a simple multiscale analysis of the ultraviolet problem. This strategy was described many times before in the literature, see for instance [3, 4, 5, 9, 15]; for completeness, it will be presented in a self-contained form in Appendix $[\mathrm{B}$. Recently, a different proof based on a single scale integration step and using improved bounds on determinants associated to "chronological products" was proposed [21].

It is important for the incoming discussion to note that the symmetries listed in Lemma 1 also imply some non trivial invariance properties of the kernels. We will be particularly interested in the invariance properties of the quadratic part $\hat{W}_{2,\left(\rho_{1}, \rho_{2}\right)}(\mathbf{k})$, which will be used below to show that the structure of the quadratic part of the new effective interaction has the same symmetries as the free integration. The crucial properties that we will need are the following.

Lemma 2. Let $\hat{W}_{a a}(\mathbf{k}) \equiv \hat{W}_{2,(1,1)}(\mathbf{k}), \hat{W}_{b b}(\mathbf{k})=\hat{W}_{2,(2,2)}(\mathbf{k}), \hat{W}_{a b}(\mathbf{k})=\hat{W}_{2,(1,2)}(\mathbf{k})$ and $\hat{W}_{b a}(\mathbf{k})=\hat{W}_{2,(2,1)}(\mathbf{k})$. Then the following properties are valid:

(i) $W_{a a}(\mathbf{k})=W_{b b}(\mathbf{k})$ and $W_{a b}(\mathbf{k})=W_{b a}^{*}(\mathbf{k})$;

(ii) as $\beta \rightarrow \infty$, for $\omega= \pm, W_{a a}\left(0, \vec{p}_{F}^{\omega}\right)=W_{a b}\left(0, \vec{p}_{F}^{\omega}\right)=0$;

(iii) as $\beta,|\Lambda| \rightarrow \infty$, for $\omega= \pm$,

$$
\begin{aligned}
& \partial_{\vec{k}} \hat{W}_{a a}\left(0, \vec{p}_{F}^{\omega}\right)=\overrightarrow{0}, \quad \operatorname{Re}\left\{\partial_{k_{0}} \hat{W}_{a a}\left(0, \vec{p}_{F}^{\omega}\right)\right\}=0, \quad \partial_{k_{0}} \hat{W}_{a b}\left(0, \vec{p}_{F}^{\omega}\right)=0, \\
& \operatorname{Re}\left\{\partial_{k_{1}} \hat{W}_{a b}\left(0, \vec{p}_{F}^{\omega}\right)\right\}=\operatorname{Im}\left\{\partial_{k_{2}} \hat{W}_{a b}\left(0, \vec{p}_{F}^{\omega}\right)\right\}=0, \quad i \partial_{k_{1}} \hat{W}_{a b}\left(0, \vec{p}_{F}^{\omega}\right)=\omega \partial_{k_{2}} \hat{W}_{a b}\left(0, \vec{p}_{F}^{\omega}\right) .
\end{aligned}
$$

\section{Remarks.}

1) For simplicity, the properties (ii) and (iii) are spelled out only in the zero temperature limit and in the thermodynamic limit; however, as it will be clear from the proof, those properties all have a finite temperature/volume counterpart.

2) Lemma 2 implies that in the vicinity of the Fermi points the kernel $W_{2,\left(\rho, \rho^{\prime}\right)}(\mathbf{k})$ can be rewritten in the form

$$
W_{2,\left(\rho, \rho^{\prime}\right)}\left(k_{0}, \vec{p}_{F}^{\omega}+\vec{k}^{\prime}\right) \simeq\left(\begin{array}{cc}
-i z_{0} k_{0} & \delta_{0}\left(i k_{1}^{\prime}-\omega k_{2}^{\prime}\right) \\
\delta_{0}\left(-i k_{1}^{\prime}-\omega k_{2}^{\prime}\right) & -i z_{0} k_{0}
\end{array}\right)_{\rho, \rho^{\prime}}
$$


for some real constants $z_{0}, \delta_{0}$, modulo higher order terms in $\left(k_{0}, \vec{k}^{\prime}\right)$. Therefore, it is apparent that its structure is the same as the one of $\hat{S}_{0}(\mathbf{k})$, modulo higher order terms in $\left(k_{0}, \vec{k}^{\prime}\right)$.

Proof. As remarked after (3.36),$P\left(d \Psi^{(u . v .)}\right)$ and $P\left(d \Psi^{(i . r .)}\right)$ are separately invariant under the symmetry properties listed in Lemma 1 . Therefore $\mathcal{V}(\Psi)$ is also invariant under the same symmetries, and so is the quadratic part of $\mathcal{V}(\Psi)$, that is

$$
\begin{aligned}
(\beta|\Lambda|)^{-2} \sum_{\sigma} & \sum_{\mathbf{k}, \mathbf{p}} \delta(\mathbf{p})\left[\hat{\Psi}_{\mathbf{k}, \sigma, 1}^{(i . r .)+} \hat{\Psi}_{\mathbf{k}+\mathbf{p}, \sigma, 1}^{(i . r)-} W_{a a}(\mathbf{k})+\hat{\Psi}_{\mathbf{k}, \sigma, 1}^{(i . r .)+} \hat{\Psi}_{\mathbf{k}+\mathbf{p}, \sigma, 2}^{(i . r .)-} W_{a b}(\mathbf{k})+\right. \\
& \left.+\hat{\Psi}_{\mathbf{k}, \sigma, 2}^{(i . r .)+} \hat{\Psi}_{\mathbf{k}+\mathbf{p}, \sigma, 1}^{(i . r)-} W_{b a}(\mathbf{k})+\hat{\Psi}_{\mathbf{k}, \sigma, 2}^{(i . r .)+} \hat{\Psi}_{\mathbf{k}+\mathbf{p}, \sigma, 2}^{(i . r .)-} W_{b b}(\mathbf{k})\right] .
\end{aligned}
$$

Recall that, as assumed in the lines preceding (3.28) , the support of $\hat{\Psi}^{(i . r .)}$ consists of two disjoint regions around $\vec{p}_{F}^{+}$and $\vec{p}_{F}$, respectively; in particular, we assumed that $2 a_{0} \gamma<4 \pi / 3-4 \pi /(3 \sqrt{3})$. Under this condition, it is easy to realize that if both $\mathbf{k}$ and $\mathbf{p}+\mathbf{k}$ belong to the support of $\hat{\Psi}^{(i . r .)}$, then $|\mathbf{p}|<4 \pi / 3$. As a consequence, in (3.37), the only non zero contributions correspond to the terms with $\mathbf{p}=\mathbf{0}$ (in fact, if $\mathbf{p}$ is $\neq \mathbf{0}$ and belongs to the support of $\delta(\mathbf{p})$, then $|\mathbf{p}| \geq 4 \pi / 3$, which means that either $\mathbf{k}$ or $\mathbf{k}+\mathbf{p}$ is outside the support of $\hat{\Psi}^{(i . r .)}$, and the corresponding term in the sum is identically zero). This means that the sum

$$
\begin{aligned}
\sum_{\sigma, \mathbf{k}}\left[\hat{\Psi}_{\mathbf{k}, \sigma, 1}^{(i . r .)+} \hat{\Psi}_{\mathbf{k}, \sigma, 1}^{(i . r .)-} W_{a a}(\mathbf{k})+\hat{\Psi}_{\mathbf{k}, \sigma, 1}^{(i . r .)+} \hat{\Psi}_{\mathbf{k}, \sigma, 2}^{(i . r .)-} W_{a b}(\mathbf{k})+\right. \\
\left.+\hat{\Psi}_{\mathbf{k}, \sigma, 2}^{(i . r .)+} \hat{\Psi}_{\mathbf{k}, \sigma, 1}^{(i . r .)-} W_{b a}(\mathbf{k})+\hat{\Psi}_{\mathbf{k}, \sigma, 2}^{(i . r .)+} \hat{\Psi}_{\mathbf{k}, \sigma, 2}^{(i . r .)-} W_{b b}(\mathbf{k})\right] .
\end{aligned}
$$

is invariant under the symmetries (1)-(7) listed in Lemma 1.

Invariance under symmetry (4) implies that:

$$
\begin{aligned}
& W_{a a}\left(k_{0}, \vec{k}\right)=W_{a a}\left(k_{0}, T_{1}^{-1} \vec{k}\right), \quad W_{b b}\left(k_{0}, \vec{k}\right)=W_{b b}\left(k_{0}, T_{1}^{-1} \vec{k}\right), \\
& W_{a b}\left(k_{0}, \vec{k}\right)=e^{i \vec{k}\left(\vec{\delta}_{1}-\vec{\delta}_{2}\right)} W_{a b}\left(k_{0}, T_{1}^{-1} \vec{k}\right), \quad W_{b a}\left(k_{0}, \vec{k}\right)=e^{-i \vec{k}\left(\vec{\delta}_{1}-\vec{\delta}_{2}\right)} W_{a b}\left(k_{0}, T_{1}^{-1} \vec{k}\right) ;
\end{aligned}
$$

invariance under (5) implies that:

$$
\begin{array}{ll}
W_{a a}(\mathbf{k})=W_{a a}(-\mathbf{k})^{*}, & W_{b b}(\mathbf{k})=W_{b b}(-\mathbf{k})^{*}, \\
W_{a b}(\mathbf{k})=W_{a b}(-\mathbf{k})^{*}, & W_{b a}(\mathbf{k})=W_{b a}(-\mathbf{k})^{*} ;
\end{array}
$$

invariance under (6.a) implies that:

$$
W_{a a}\left(k_{0}, k_{1}, k_{2}\right)=W_{b b}\left(k_{0},-k_{1}, k_{2}\right), \quad W_{a b}\left(k_{0}, k_{1}, k_{2}\right)=W_{b a}\left(k_{0},-k_{1}, k_{2}\right) ;
$$

invariance under (6.b) implies that:

$$
\begin{aligned}
& W_{a a}\left(k_{0}, k_{1}, k_{2}\right)=W_{a a}\left(k_{0}, k_{1},-k_{2}\right), \quad W_{b b}\left(k_{0}, k_{1}, k_{2}\right)=W_{b b}\left(k_{0}, k_{1},-k_{2}\right), \\
& W_{a b}\left(k_{0}, k_{1}, k_{2}\right)=W_{a b}\left(k_{0}, k_{1},-k_{2}\right), \quad W_{b a}\left(k_{0}, k_{1}, k_{2}\right)=W_{b a}\left(k_{0}, k_{1},-k_{2}\right) ;
\end{aligned}
$$

invariance under (7) implies that:

$$
\begin{aligned}
& W_{a a}\left(k_{0}, \vec{k}\right)=W_{a a}\left(k_{0},-\vec{k}\right), \quad W_{b b}\left(k_{0}, \vec{k}\right)=W_{b b}\left(k_{0},-\vec{k}\right), \\
& W_{a b}\left(k_{0}, \vec{k}\right)=W_{b a}\left(k_{0},-\vec{k}\right) ;
\end{aligned}
$$

Finally, invariance under (8) implies that:

$$
\begin{aligned}
& W_{a a}\left(k_{0}, \vec{k}\right)=-W_{a a}\left(-k_{0}, \vec{k}\right), \quad W_{b b}\left(k_{0}, \vec{k}\right)=-W_{b b}\left(-k_{0}, \vec{k}\right), \\
& W_{a b}\left(k_{0}, \vec{k}\right)=W_{a b}\left(-k_{0}, \vec{k}\right), \quad W_{b a}\left(k_{0}, \vec{k}\right)=W_{b a}\left(-k_{0}, \vec{k}\right) ;
\end{aligned}
$$

Now, combining the first of (3.44), the second of (3.45) and the second of (3.46), we find that $W_{a a}(\mathbf{k})=W_{b b}(\mathbf{k})$. Combining the third of (3.43), the third of (3.46) and the last of (3.47), we find that $W_{a b}(\mathbf{k})=W_{b a}(\mathbf{k})^{*}$. This concludes the proof of item (i). 
The first of (3.47) implies that, as $\beta \rightarrow \infty, W_{a a}(0, \vec{k})=0$, and this proves, in particular, that $W_{a a}\left(0, \vec{p}_{F}^{\omega}\right)=0$ and that, in the limit $|\Lambda| \rightarrow \infty, \partial_{\vec{k}} W_{a a}\left(0, \vec{p}_{F}^{\omega}\right)=\overrightarrow{0}$.

Using that $\vec{p}_{F}^{\omega}$ is invariant under the action of $T_{1}$, we see that the third of (3.42) implies that $\left(1-e^{i \vec{p}_{F}^{\omega}\left(\vec{\delta}_{1}-\vec{\delta}_{2}\right)}\right) W_{a b}\left(k_{0}, \vec{p}_{F}^{\omega}\right)=0$. Since $e^{i \vec{p}_{F}^{\omega}\left(\vec{\delta}_{1}-\vec{\delta}_{2}\right)}=-e^{i \omega \pi / 3} \neq 1$, this identity proves, in particular, that $W_{a b}\left(0, \vec{p}_{F}^{\omega}\right)=0$, and $\partial_{k_{0}} W_{a b}\left(0, \vec{p}_{F}^{\omega}\right)=0$. This concludes the proof of item (ii).

Now, combining the first of (3.43) with the first of (3.46), we find that $W_{a a}\left(k_{0}, \vec{k}\right)=$ $W_{a a}\left(-k_{0}, \vec{k}\right)^{*}$, which implies, in particular, that $\operatorname{Re}\left\{\partial_{k_{0}} \hat{W}_{a a}\left(0, \vec{p}_{F}^{\omega}\right)\right\}=0$.

Finally, let $W_{a b}\left(0, \vec{p}_{F}^{\omega}+\vec{k}^{\prime}\right) \simeq \alpha_{1}^{\omega} k_{1}^{\prime}+\alpha_{2}^{\omega} k_{2}^{\prime}$, modulo higher order terms in $\vec{k}^{\prime}$. Using that $T_{1}^{-1}=\left(\begin{array}{cc}-1 / 2 & \sqrt{3} / 2 \\ -\sqrt{3} / 2 & -1 / 2\end{array}\right)$ in the third of (3.42), we find that

$$
\alpha_{1}^{\omega} k_{1}^{\prime}+\alpha_{2}^{\omega} k_{2}^{\prime}=e^{-i \omega \pi / 3}\left[\alpha_{1}^{\omega}\left(k_{1}^{\prime} / 2-\sqrt{3} k_{2}^{\prime} / 2\right)+\alpha_{2}^{\omega}\left(\sqrt{3} k_{1}^{\prime} / 2+k_{2}^{\prime} / 2\right)\right],
$$

which implies $\alpha_{1}^{\omega}=-i \omega \alpha_{2}^{\omega}$. Moreover, using the third of (3.43) we find that $\alpha_{i}^{\omega}=-\left(\alpha_{i}^{-\omega}\right)^{*}$, and using the third of (3.45) we find that $\alpha_{2}^{\omega}=-\alpha_{2}^{-\omega}$. Therefore, $\alpha_{2}^{\omega}=-\alpha_{2}^{-\omega}=-\left(\alpha_{2}^{-\omega}\right)^{*}$, and we see that $\alpha_{2}^{\omega}$ is real and odd in $\omega$, that is $\alpha_{2}^{\omega}=\omega a$, for some real constant $a$. Therefore, $\alpha_{1}^{\omega}=-i \omega \alpha_{2}^{\omega}=-i a$, and this concludes the proof of item (iii).

\section{Free energy: The infrared integration}

Multiscale analysis. In order to compute (3.33) we shall proceed in an iterative fashion, using standard functional Renormalization Group methods [2, 13, 18]. As a starting point, it is convenient to decompose the infrared propagator as:

$$
g^{(i . r .)}(\mathbf{x}, \mathbf{y})=\sum_{\omega= \pm} e^{-i \vec{p}_{F}^{\omega}(\vec{x}-\vec{y})} g_{\omega}^{(\leq 0)}(\mathbf{x}, \mathbf{y})
$$

where, if $\mathbf{k}^{\prime}=\left(k_{0}, \vec{k}^{\prime}\right)$,

$$
g_{\omega}^{(\leq 0)}(\mathbf{x}, \mathbf{y})=\frac{1}{\beta|\Lambda|} \sum_{\mathbf{k}^{\prime} \in \mathcal{D}_{\beta, L}^{\omega}} \chi_{0}\left(\left|\mathbf{k}^{\prime}\right|\right) e^{-i \mathbf{k}^{\prime}(\mathbf{x}-\mathbf{y})}\left(\begin{array}{cc}
-i k_{0} & -v^{*}\left(\vec{k}^{\prime}+\vec{p}_{F}^{\omega}\right) \\
-v\left(\vec{k}^{\prime}+\vec{p}_{F}^{\omega}\right) & -i k_{0}
\end{array}\right)^{-1}
$$

and $\mathcal{D}_{\beta, L}^{\omega}=\mathcal{D}_{\beta} \times \mathcal{D}_{L}^{\omega}$, with $\mathcal{D}_{L}^{\omega}=\left\{\frac{n_{1}}{L} \vec{b}_{1}+\frac{n_{2}}{L} \vec{b}_{2}-\frac{(L \mid \bmod 3)}{L} \vec{p}_{F}^{\omega},\left[\frac{-L}{2}\right]+1 \leq n_{1}, n_{2} \leq\left[\frac{L}{2}\right]\right\}$.

Correspondingly, we rewrite $\Psi^{(i . r .)}$ as a sum of two independent Grassmann fields:

$$
\Psi_{\mathbf{x}, \sigma, \rho}^{(i . r .) \pm}=\sum_{\omega= \pm} e^{i \vec{p}_{F}^{\omega} \vec{x}} \Psi_{\mathbf{x}, \sigma, \rho, \omega}^{(\leq 0) \pm}
$$

and we rewrite $(3.33)$ in the form:

$$
\Xi_{\beta, L}=e^{-\beta|\Lambda| F_{0}} \int P_{\chi_{0}, A_{0}}\left(d \Psi^{(\leq 0)}\right) e^{-\mathcal{V}^{(0)}\left(\Psi^{(\leq 0)}\right)},
$$

where $\mathcal{V}^{(0)}\left(\Psi^{(\leq 0)}\right)$ is equal to $\mathcal{V}\left(\Psi^{(i . r .)}\right)$, once $\Psi^{(i . r .)}$ is rewritten as in (3.51), i.e.,

$$
\begin{gathered}
\mathcal{V}^{(0)}\left(\Psi^{(\leq 0)}\right)= \\
=\sum_{n=1}^{\infty}(\beta|\Lambda|)^{-2 n} \sum_{\sigma_{1}, \ldots, \sigma_{n}=\uparrow \downarrow} \sum_{\rho_{1}, \ldots, \rho_{2 n}=1,2}^{\omega_{1}, \ldots, \omega_{2 n}= \pm} \sum_{\mathbf{k}_{1}^{\prime}, \ldots, \mathbf{k}_{2 n}^{\prime}}\left[\prod_{j=1}^{n} \hat{\Psi}_{\mathbf{k}_{2 j-1}^{\prime}, \sigma_{j}, \rho_{2 j-1}, \omega_{2 j-1}}^{(\leq 0)+} \hat{\Psi}_{\mathbf{k}_{2 j}^{\prime}, \sigma_{j}, \rho_{2 j}, \omega_{2 j}}^{(\leq 0)-}\right] . \\
\cdot \hat{W}_{2 n, \underline{\rho}, \underline{\omega}}^{(0)}\left(\mathbf{k}_{1}^{\prime}, \ldots, \mathbf{k}_{2 n-1}^{\prime}\right) \delta\left(\sum_{j=1}^{2 n}(-1)^{j}\left(\mathbf{p}_{F}^{\omega_{j}}+\mathbf{k}_{j}^{\prime}\right)\right)= \\
=\sum_{n=1}^{\infty} \sum_{\underline{\sigma}, \underline{\rho}, \underline{\omega}}^{n} \int d \mathbf{x}_{1} \cdots d \mathbf{x}_{2 n}\left[\prod_{j=1}^{n} \Psi_{\mathbf{x}_{2 j-1}, \sigma_{j}, \rho_{2 j-1}, \omega_{2 j-1}}^{(\leq 0)+} \Psi_{\mathbf{x}_{2 j}, \sigma_{j}, \rho_{2 j}, \omega_{2 j}}^{(\leq 0)-}\right] W_{2 n, \underline{\rho}, \underline{\omega}}^{(0)}\left(\mathbf{x}_{1}, \ldots, \mathbf{x}_{2 n}\right),
\end{gathered}
$$


with:

1) $\underline{\omega}=\left(\omega_{1}, \ldots, \omega_{2 n}\right), \underline{\sigma}=\left(\sigma_{1}, \ldots, \sigma_{n}\right)$ and $\mathbf{p}_{F}^{\omega}=\left(0, \vec{p}_{F}^{\omega}\right)$;

2) $\hat{W}_{2 n, \underline{\rho}, \underline{\omega}}^{(0)}\left(\mathbf{k}_{1}^{\prime}, \ldots, \mathbf{k}_{2 n-1}^{\prime}\right)=\hat{W}_{2 n, \underline{\rho}}\left(\mathbf{k}_{1}^{\prime}+\mathbf{p}_{F}^{\omega_{j}}, \ldots, \mathbf{k}_{2 n-1}^{\prime}+\mathbf{p}_{F}^{\omega_{2 n-1}}\right)$, see (3.35);

$3)$ the kernels $W_{2 n, \underline{\rho}, \underline{\omega}}^{(0)}\left(\mathbf{x}_{1}, \ldots, \mathbf{x}_{2 n}\right)$ are defined as:

$$
\begin{aligned}
& W_{2 n, \underline{\rho}, \underline{\omega}}^{(0)}\left(\mathbf{x}_{1}, \ldots, \mathbf{x}_{2 n}\right)= \\
& =(\beta|\Lambda|)^{-2 n} \sum_{\mathbf{k}_{1}^{\prime}, \ldots, \mathbf{k}_{2 n}^{\prime}} e^{i \sum_{j=1}^{2 n}(-1)^{j} \mathbf{k}_{j} \mathbf{x}_{j}} \hat{W}_{2 n, \underline{\rho}, \underline{\omega}}^{(0)}\left(\mathbf{k}_{1}^{\prime}, \ldots, \mathbf{k}_{2 n-1}^{\prime}\right) \delta\left(\sum_{j=1}^{2 n}(-1)^{j}\left(\mathbf{p}_{F}^{\omega_{j}}+\mathbf{k}_{j}^{\prime}\right)\right) .
\end{aligned}
$$

Moreover, $P_{\chi_{0}, A_{0}}\left(d \Psi^{(\leq 0)}\right)$ is defined as

$$
\begin{aligned}
P_{\chi_{0}, A_{0}}\left(d \Psi^{(\leq 0)}\right)=\mathcal{N}_{0}^{-1}\left[\prod_{\mathbf{k}^{\prime} \in \mathcal{D}_{\beta, L}^{\omega}}^{\chi_{0}\left(\left|\mathbf{k}^{\prime}\right|\right)>0} \prod_{\sigma, \omega, \rho} d \hat{\Psi}_{\mathbf{k}^{\prime}, \sigma, \rho, \omega}^{(\leq 0)+} d \hat{\Psi}_{\mathbf{k}^{\prime}, \sigma, \rho, \omega}^{(\leq 0)-}\right] \\
\cdot \exp \left\{-(\beta|\Lambda|)^{-1} \sum_{\omega= \pm, \sigma=\uparrow \downarrow} \sum_{\mathbf{k}^{\prime} \in \mathcal{D}_{\beta, L}^{\omega}}^{\chi_{0}\left(\left|\mathbf{k}^{\prime}\right|\right)>0} \chi_{0}^{-1}\left(\left|\mathbf{k}^{\prime}\right|\right) \hat{\Psi}_{\mathbf{k}^{\prime}, \sigma, \cdot, \omega}^{(\leq 0)+} A_{0, \omega}\left(\mathbf{k}^{\prime}\right) \hat{\Psi}_{\mathbf{k}^{\prime}, \sigma, \cdot, \omega}^{(\leq 0)-}\right\},
\end{aligned}
$$

where:

$$
\begin{aligned}
A_{0, \omega}\left(\mathbf{k}^{\prime}\right) & =\left(\begin{array}{cc}
-i k_{0} & -v^{*}\left(\vec{k}^{\prime}+\vec{p}_{F}^{\omega}\right) \\
-v\left(\vec{k}^{\prime}+\vec{p}_{F}^{\omega}\right) & -i k_{0}
\end{array}\right)= \\
& =\left(\begin{array}{cc}
-i \zeta_{0} k_{0}+s_{0}\left(\mathbf{k}^{\prime}\right) & c_{0}\left(i k_{1}^{\prime}-\omega k_{2}^{\prime}\right)+t_{0, \omega}\left(\mathbf{k}^{\prime}\right) \\
c_{0}\left(-i k_{1}^{\prime}-\omega k_{2}^{\prime}\right)+t_{0, \omega}^{*}\left(\mathbf{k}^{\prime}\right) & -i \zeta_{0} k_{0}+s_{0}\left(\mathbf{k}^{\prime}\right)
\end{array}\right),
\end{aligned}
$$

$\mathcal{N}_{0}$ is chosen in such a way that $\int P_{\chi_{0}, A_{0}}\left(d \Psi^{(\leq 0)}\right)=1, \zeta_{0}=1, c_{0}=3 / 2, s_{0} \equiv 0$ and $\left|t_{0, \omega}\left(\mathbf{k}^{\prime}\right)\right| \leq$ $C\left|\mathbf{k}^{\prime}\right|^{2}$

It is apparent that the $\Psi^{(\leq 0)}$ field has zero mass (i.e., its propagator decays polynomially at large distances in $\mathbf{x}$-space). Therefore, its integration requires an infrared multiscale analysis. We consider the scaling parameter $\gamma>1$ introduced above, see the lines preceding (3.28), and we define a sequence of geometrically decreasing momentum scales $\gamma^{h}, h=0,-1,-2, \ldots$ Correspondingly we introduce compact support functions $f_{h}\left(\mathbf{k}^{\prime}\right)=\chi_{0}\left(\gamma^{-h}\left|\mathbf{k}^{\prime}\right|\right)-\chi_{0}\left(\gamma^{-h+1}\left|\mathbf{k}^{\prime}\right|\right)$ and we rewrite

$$
\chi_{0}\left(\left|\mathbf{k}^{\prime}\right|\right)=\sum_{h=-\infty}^{0} f_{h}\left(\mathbf{k}^{\prime}\right) .
$$

The purpose is to perform the integration of (3.52) in an iterative way. We step by step decompose the propagator into a sum of two propagators, the first supported on momenta $\sim \gamma^{h}, h \leq 0$, the second supported on momenta smaller than $\gamma^{h}$. Correspondingly we rewrite the Grassmann field as a sum of two independent fields: $\Psi^{(\leq h)}=\Psi^{(h)}+\Psi^{(\leq h-1)}$ and we integrate the field $\Psi^{(h)}$. In this way we inductively prove that, for any $h \leq 0$, (3.52) can be rewritten as

$$
\Xi_{\beta, L}=e^{-\beta|\Lambda| F_{h}} \int P_{\chi_{h}, A_{h}}\left(d \Psi^{(\leq h)}\right) e^{-\mathcal{V}^{(h)}\left(\Psi^{(\leq h)}\right)},
$$

where $F_{h}, A_{h}, \mathcal{V}^{(h)}$ will be defined recursively, $\chi_{h}\left(\left|\mathbf{k}^{\prime}\right|\right)=\sum_{k=-\infty}^{h} f_{k}\left(\mathbf{k}^{\prime}\right)$ and $P_{\chi_{h}, A_{h}}\left(d \Psi^{(\leq h)}\right)$ is defined in the same way as $P_{\chi_{0}, A_{0}}\left(d \Psi^{(\leq 0)}\right)$ with $\Psi^{(\leq 0)}, \chi_{0}, A_{0, \omega}, \zeta_{0}, c_{0}, s_{0}, t_{0, \omega}$ replaced by $\Psi^{(\leq h)}, \chi_{h}, A_{h, \omega}, \zeta_{h}, c_{h}, s_{h}, t_{h, \omega}$, respectively. Moreover $\mathcal{V}^{(h)}(0)=0$ and

$$
\begin{gathered}
\mathcal{V}^{(h)}(\Psi)=\sum_{n=1}^{\infty}(\beta|\Lambda|)^{-2 n} \sum_{\underline{\sigma}, \underline{\rho}, \underline{\omega}} \sum_{\mathbf{k}_{1}^{\prime}, \ldots, \mathbf{k}_{2 n}^{\prime}}\left[\prod_{j=1}^{n} \hat{\Psi}_{\mathbf{k}_{2 j-1}^{\prime}, \sigma_{j}, \rho_{2 j-1}, \omega_{2 j-1}}^{(\leq h)+} \hat{\Psi}_{\mathbf{k}_{2 j}^{\prime}, \sigma_{j}, \rho_{2 j}, \omega_{2 j}}^{(\leq h)-} .\right. \\
\cdot \hat{W}_{2 n, \underline{\rho}, \underline{\omega}}^{(h)}\left(\mathbf{k}_{1}^{\prime}, \ldots, \mathbf{k}_{2 n-1}^{\prime}\right) \delta\left(\sum_{j=1}^{2 n}(-1)^{j}\left(\mathbf{p}_{F}^{\omega_{j}}+\mathbf{k}_{j}^{\prime}\right)\right)=
\end{gathered}
$$




$$
=\sum_{n=1}^{\infty} \sum_{\underline{\sigma}, \underline{\rho}, \underline{\omega}} \int d \mathbf{x}_{1} \cdots d \mathbf{x}_{2 n}\left[\prod_{j=1}^{n} \Psi_{\mathbf{x}_{2 j-1}, \sigma_{j}, \rho_{2 j-1}, \omega_{2 j-1}}^{(\leq h)+} \Psi_{\mathbf{x}_{2 j}, \sigma_{j}, \rho_{2 j}, \omega_{2 j}}^{(\leq h)-}\right] W_{2 n, \underline{\rho}, \underline{\omega}}^{(h)}\left(\mathbf{x}_{1}, \ldots, \mathbf{x}_{2 n}\right) .
$$

Note that the field $\Psi_{\mathbf{k}^{\prime}, \sigma, \cdot \omega}^{(\leq h)}$, whose propagator is given by $\chi_{h}\left(\left|\mathbf{k}^{\prime}\right|\right)\left[A_{\omega}^{(h)}\left(\mathbf{k}^{\prime}\right)\right]^{-1}$, has the same support as $\chi_{h}$, that is on a neighborood of size $\gamma^{h}$ around the singularity $\mathbf{k}^{\prime}=\mathbf{0}$ (that, in the original variables, corresponds to the Dirac point $\mathbf{k}=\mathbf{p}_{F}^{\omega}$ ). It is important for the following to think $\hat{W}_{2 n, \underline{\rho}, \underline{\omega}}^{(h)}, h \leq 0$, as functions of the variables $\left\{\zeta_{k}, c_{k}\right\}_{h<k \leq 0}$. The iterative construction below will inductively imply that the dependence on these variables is well defined.

The iteration will continue up to the scale $h_{\beta}$, where $h_{\beta}$ is the largest scale such that

$$
a_{0} \gamma^{h_{\beta}-1}<\frac{\pi}{\beta} \zeta_{h_{\beta}}
$$

where $a_{0}$ is the constant appearing in the definition of $\chi_{0}\left(\left|\mathbf{k}^{\prime}\right|\right)$. By the properties of $\zeta_{h}$ that will be described and proved below, it will turn out that $h_{\beta}$ is finite and larger than $\log _{\gamma} \frac{\pi}{2 a_{0} \beta}$. The result of the last iteration will be $\Xi_{\beta, L}$, i.e., the value of the partition function.

Localization and renormalization. In order to inductively prove (3.57) we write

$$
\mathcal{V}^{(h)}=\mathcal{L} \mathcal{V}^{(h)}+\mathcal{R} \mathcal{V}^{(h)}
$$

where

$$
\mathcal{L} \mathcal{V}^{(h)}=\frac{1}{\beta|\Lambda|} \sum_{\sigma_{1}, \sigma_{2}=\uparrow \downarrow} \sum_{\substack{\rho_{1}, \rho_{2}=1,2 \\ \omega= \pm}} \sum_{\mathbf{k}^{\prime}}^{\chi_{h}\left(\left|\mathbf{k}^{\prime}\right|\right)>0} \hat{\Psi}_{\mathbf{k}^{\prime}, \sigma_{1}, \rho_{1}, \omega}^{(\leq h)+} \hat{\Psi}_{\mathbf{k}^{\prime}, \sigma_{2}, \rho_{2}, \omega}^{(\leq h)-} \hat{W}_{2, \underline{\rho},(\omega, \omega)}^{(h)}\left(\mathbf{k}^{\prime}\right)
$$

and $\mathcal{R} \mathcal{V}^{(h)}$ is given by $(3.58)$ with $\sum_{n=1}^{\infty}$ replaced by $\sum_{n=2}^{\infty}$, that is it contains only the monomials with more than four fields.

Note that in (3.61) the $\omega$-index of the $\Psi$ fields is the same; this follows from the fact that in the terms with different $\omega$ 's the momenta verify $\mathbf{k}_{1}^{\prime}-\mathbf{k}_{2}^{\prime}+\mathbf{p}_{F}^{\omega}-\mathbf{p}_{F}^{-\omega}=n_{1} \vec{b}_{1}+n_{2} \vec{b}_{2}$, for some choice of $n_{1}, n_{2}$, and such a condition cannot be verified if $\mathbf{k}_{1}^{\prime}, \mathbf{k}_{2}^{\prime}$ are in the support of the $\Psi^{(\leq h)}$ fields, because $\mathbf{p}_{F}^{\omega}-\mathbf{p}_{F}^{-\omega} \notin \Lambda^{*}$ and $2 a_{0} \gamma$ is smaller than $4 \pi / 3-4 \pi /(3 \sqrt{3})$, see the lines preceding (3.28) and the discussion after (3.40).

Remark. The fact that the quadratic terms with different $\omega$ 's, i.e., the one particle umklapp processes, do not contribute to the infrared effective potential is a crucial fact, which reduces the number of relevant running coupling constants and, in particular, tells us that the interaction does not generate mass terms. Note, in fact, that the presence of one particle umklapp terms with a non zero contribution at the Fermi points could produce an exponential decay of the interacting correlations.

The symmetries of the action, listed in Lemma 1, which are preserved by the iterative integration procedure, imply that, in the zero temperature and thermodynamic limit, $\hat{W}_{2, \underline{\rho},(\omega, \omega)}^{(h)}(\mathbf{0})=0$ and

$$
\mathbf{k}^{\prime} \partial_{\mathbf{k}^{\prime}} \hat{W}_{2,\left(\rho_{1}, \rho_{2}\right),(\omega, \omega)}^{(h)}(\mathbf{0})=\left(\begin{array}{cc}
-i z_{h} k_{0} & \delta_{h}\left(i k_{1}^{\prime}-\omega k_{2}^{\prime}\right) \\
\delta_{h}\left(-i k_{1}^{\prime}-\omega k_{2}^{\prime}\right) & -i z_{h} k_{0}
\end{array}\right)_{\rho_{1}, \rho_{2}}
$$

for suitable real constants $z_{h}, \delta_{h}$. The proof of (3.62) is completely analogous to the proof of Lemma 2 and will not be repeated here.

Once that the above definitions are given, we can describe our iterative integration procedure for $h \leq 0$. We start from (3.57) and we rewrite it as

$$
\int P_{\chi_{h}, A_{h}}\left(d \Psi^{(\leq h)}\right) e^{-\mathcal{L} \mathcal{V}^{(h)}\left(\Psi^{(\leq h)}\right)-\mathcal{R} \mathcal{V}^{(h)}\left(\Psi^{(\leq h)}\right)-\beta|\Lambda| F_{h}},
$$


with

$$
\begin{aligned}
\mathcal{L} \mathcal{V}^{(h)}\left(\Psi^{(\leq h)}\right) & =(\beta|\Lambda|)^{-1} \sum_{\omega, \sigma} \sum_{\mathbf{k}^{\prime}}^{\chi_{h}\left(\left|\mathbf{k}^{\prime}\right|\right)>0} \\
& \cdot \hat{\Psi}_{\mathbf{k}^{\prime}, \sigma, \cdot, \omega}^{(\leq h)+}\left(\begin{array}{cc}
-i z_{h} k_{0}+\sigma_{h}\left(\mathbf{k}^{\prime}\right) & \delta_{h}\left(i k_{1}^{\prime}-\omega k_{2}^{\prime}\right)+\tau_{h, \omega}\left(\mathbf{k}^{\prime}\right) \\
\delta_{h}\left(-i k_{1}^{\prime}-\omega k_{2}\right)+\tau_{h, \omega}^{*}\left(\mathbf{k}^{\prime}\right) & -i z_{h} k_{0}+\sigma_{h}\left(\mathbf{k}^{\prime}\right)
\end{array}\right) \hat{\Psi}_{\mathbf{k}^{\prime}, \sigma, \cdot, \omega}^{(\leq h)-}
\end{aligned}
$$

Then we include $\mathcal{L} \mathcal{V}^{(h)}$ in the fermionic integration, so obtaining

$$
\int P_{\chi_{h}, \bar{A}_{h-1}}\left(d \Psi^{(\leq h)}\right) e^{-\mathcal{R} \mathcal{V}^{(h)}\left(\Psi^{(\leq h)}\right)-\beta|\Lambda|\left(F_{h}+e_{h}\right)}
$$

where $e_{h}$ is a constant that takes into account the change in the normalization factor of the measure and

$$
\bar{A}_{h-1, \omega}\left(\mathbf{k}^{\prime}\right)=\left(\begin{array}{cc}
-i \bar{\zeta}_{h-1} k_{0}+\bar{s}_{h-1}\left(\mathbf{k}^{\prime}\right) & \bar{c}_{h-1}\left(i k_{1}^{\prime}-\omega k_{2}^{\prime}\right)+\bar{t}_{h-1, \omega}\left(\mathbf{k}^{\prime}\right) \\
\bar{c}_{h-1}\left(-i k_{1}^{\prime}-\omega k_{2}^{\prime}\right)+\bar{t}_{h-1, \omega}^{*}\left(\mathbf{k}^{\prime}\right) & -i \bar{\zeta}_{h-1} k_{0}+\bar{s}_{h-1}\left(\mathbf{k}^{\prime}\right)
\end{array}\right)
$$

with:

$$
\begin{aligned}
& \bar{\zeta}_{h-1}\left(\mathbf{k}^{\prime}\right)=\zeta_{h}+z_{h} \chi_{h}\left(\mathbf{k}^{\prime}\right), \quad \bar{c}_{h-1}\left(\mathbf{k}^{\prime}\right)=c_{h}+\delta_{h} \chi_{h}\left(\mathbf{k}^{\prime}\right) \\
& \bar{s}_{h-1}\left(\mathbf{k}^{\prime}\right)=s_{h}\left(\mathbf{k}^{\prime}\right)+\sigma_{h}\left(\mathbf{k}^{\prime}\right) \chi_{h}\left(\mathbf{k}^{\prime}\right), \quad \bar{t}_{h-1, \omega}\left(\mathbf{k}^{\prime}\right)=t_{h, \omega}\left(\mathbf{k}^{\prime}\right)+\tau_{h, \omega}\left(\mathbf{k}^{\prime}\right) \chi_{h}\left(\mathbf{k}^{\prime}\right) \text {. }
\end{aligned}
$$

Now we can perform the integration of the $\Psi^{(h)}$ field. We rewrite the Grassmann field $\Psi^{(\leq h)}$ as a sum of two independent Grassmann fields $\Psi^{(\leq h-1)}+\Psi^{(h)}$ and correspondingly we rewrite (3.65) as

$$
e^{-\beta|\Lambda|\left(F_{h}+e_{h}\right)} \int P_{\chi_{h-1}, A_{h-1}}\left(d \Psi^{(\leq h-1)}\right) \int P_{f_{h}^{-1}, \bar{A}_{h-1}}\left(d \Psi^{(h)}\right) e^{-\mathcal{R} \mathcal{V}^{(h)}\left(\Psi^{(\leq h-1)}+\Psi^{(h)}\right)},
$$

where

$$
A_{h-1, \omega}\left(\mathbf{k}^{\prime}\right)=\left(\begin{array}{cc}
-i \zeta_{h-1} k_{0}+s_{h-1}\left(\mathbf{k}^{\prime}\right) & c_{h-1}\left(i k_{1}^{\prime}-\omega k_{2}^{\prime}\right)+t_{h-1, \omega}\left(\mathbf{k}^{\prime}\right) \\
c_{h-1}\left(-i k_{1}^{\prime}-\omega k_{2}^{\prime}\right)+t_{h-1, \omega}^{*}\left(\mathbf{k}^{\prime}\right) & -i \zeta_{h-1} k_{0}+s_{h-1}\left(\mathbf{k}^{\prime}\right)
\end{array}\right)
$$

with:

$$
\begin{aligned}
& \zeta_{h-1}=\zeta_{h}+z_{h}, \quad c_{h-1}=c_{h}+\delta_{h}, \\
& s_{h-1}\left(\mathbf{k}^{\prime}\right)=s_{h}\left(\mathbf{k}^{\prime}\right)+\sigma_{h}\left(\mathbf{k}^{\prime}\right), \quad t_{h-1, \omega}\left(\mathbf{k}^{\prime}\right)=t_{h, \omega}\left(\mathbf{k}^{\prime}\right)+\tau_{h, \omega}\left(\mathbf{k}^{\prime}\right) .
\end{aligned}
$$

The single scale propagator is

$$
\int P_{f_{h}^{-1}, \bar{A}_{h-1}}\left(d \Psi^{(h)}\right) \Psi_{\mathbf{x}_{1}, \sigma_{1}, \rho_{1}, \omega_{1}}^{(h)} \Psi_{\mathbf{x}_{2}, \sigma_{2}, \rho_{2}, \omega_{2}}^{(h)+}=\delta_{\sigma_{1}, \sigma_{2}} \delta_{\omega_{1}, \omega_{2}}\left[g_{\omega}^{(h)}\left(\mathbf{x}_{1}, \mathbf{x}_{2}\right)\right]_{\rho_{1}, \rho_{2}},
$$

where

$$
g_{\omega}^{(h)}\left(\mathbf{x}_{1}, \mathbf{x}_{2}\right)=\frac{1}{\beta|\Lambda|} \sum_{\mathbf{k}^{\prime} \in \mathcal{D}_{\beta, L}^{\omega}} e^{-i \mathbf{k}^{\prime}\left(\mathbf{x}_{1}-\mathbf{x}_{2}\right)} f_{h}\left(\mathbf{k}^{\prime}\right)\left[\bar{A}_{h-1, \omega}\left(\mathbf{k}^{\prime}\right)\right]^{-1} .
$$

After the integration of the field on scale $h$ we are left with an integral involving the fields $\Psi^{(\leq h-1)}$ and the new effective interaction $\mathcal{V}^{(h-1)}$, defined as

$$
e^{-\mathcal{V}^{(h-1)}\left(\Psi^{(\leq h-1)}\right)-\bar{e}_{h} \beta|\Lambda|}=\int P_{f_{h}^{-1}, \bar{A}_{h-1}}\left(d \Psi^{(h)}\right) e^{-\mathcal{R} \mathcal{V}^{(h)}\left(\Psi^{(\leq h-1)}+\Psi^{(h)}\right)} .
$$

It is easy to see that $\mathcal{V}^{(h-1)}$ is of the form (3.58) and that $F_{h-1}=F_{h}+e_{h}+\bar{e}_{h}$. It is sufficient to use the well known identity

$$
\bar{e}_{h}+\mathcal{V}^{(h-1)}\left(\Psi^{(\leq h-1)}\right)=\sum_{n \geq 1} \frac{1}{n !}(-1)^{n+1} \mathcal{E}_{h}^{T}\left(\mathcal{R V}^{(h)}\left(\Psi^{(\leq h-1)}+\Psi^{(h)}\right) ; n\right),
$$


where $\mathcal{E}_{h}^{T}\left(X\left(\Psi^{(h)}\right) ; n\right)$ is the truncated expectation of order $n$ w.r.t. the propagator $g_{\omega}^{(h)}$, which is the analogue of (3.34) with $\Psi^{(u . v .)}$ replaced by $\Psi^{(h)}$ and with $P\left(d \Psi^{(u . v .)}\right)$ replaced by $P_{f_{h}^{-1}, \bar{A}_{h-1}}\left(d \Psi^{(h)}\right)$.

Note that the above procedure allows us to write the effective renormalizations $\vec{v}_{h}=\left(\zeta_{h}, c_{h}\right)$, $h \leq 0$, in terms of $\vec{v}_{k}, h<k \leq 0$, namely $\vec{v}_{h-1}=\beta_{h}\left(\vec{v}_{h}, \ldots, \vec{v}_{0}\right)$, where $\beta_{h}$ is the so-called Beta function.

Tree expansion for the effective potentials. An iterative implementation of (3.74) leads to a representation of $\mathcal{V}^{(h)}\left(\Psi^{(\leq h)}\right)$ in terms of a tree expansion, defined as follows.

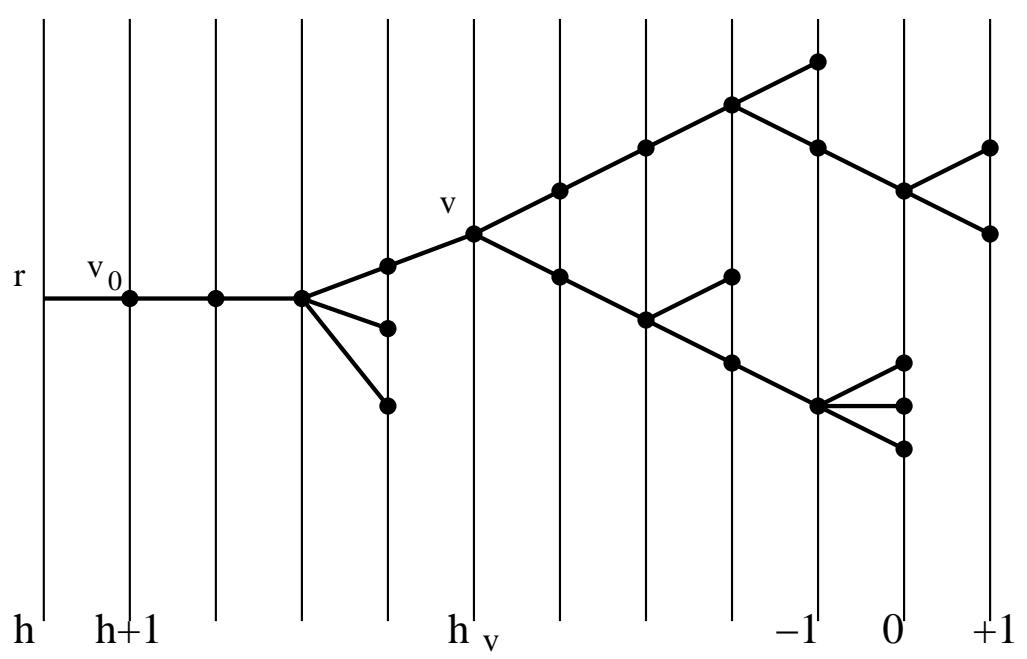

FIG. 1: A tree $\tau \in \mathcal{T}_{h, n}$ with its scale labels.

1) Let us consider the family of all trees which can be constructed by joining a point $r$, the root, with an ordered set of $n \geq 1$ points, the endpoints of the unlabeled tree, so that $r$ is not a branching point. $n$ will be called the order of the unlabeled tree and the branching points will be called the non trivial vertices. The unlabeled trees are partially ordered from the root to the endpoints in the natural way; we shall use the symbol $<$ to denote the partial order. Two unlabeled trees are identified if they can be superposed by a suitable continuous deformation, so that the endpoints with the same index coincide. It is then easy to see that the number of unlabeled trees with $n$ end-points is bounded by $4^{n}$. We shall also consider the labelled trees (to be called simply trees in the following); they are defined by associating some labels with the unlabelled trees, as explained in the following items.

2) We associate a label $h \leq-1$ with the root and we denote $\mathcal{T}_{h, n}$ the corresponding set of labeled trees with $n$ endpoints. Moreover, we introduce a family of vertical lines, labeled by an integer taking values in $[h, 1]$, and we represent any tree $\tau \in \mathcal{T}_{h, n}$ so that, if $v$ is an endpoint or a non trivial vertex, it is contained in a vertical line with index $h_{v}>h$, to be called the scale of $v$, while the root $r$ is on the line with index $h$. In general, the tree will intersect the vertical lines in set of points different from the root, the endpoints and the branching points; these points will be called trivial vertices. The set of the vertices will be the union of the endpoints, of the trivial vertices and of the non trivial vertices; note that the root is not a vertex. Every vertex $v$ of a tree will be associated to its scale label $h_{v}$, defined, as above, as the label of the vertical line whom $v$ belongs to. Note that, if $v_{1}$ and $v_{2}$ are two vertices and $v_{1}<v_{2}$, then $h_{v_{1}}<h_{v_{2}}$.

3) There is only one vertex immediately following the root, which will be denoted $v_{0}$ and cannot be an endpoint; its scale is $h+1$.

4) Given a vertex $v$ of $\tau \in \mathcal{T}_{h, n}$ that is not an endpoint, we can consider the subtrees of $\tau$ with root $v$, which correspond to the connected components of the restriction of $\tau$ to the vertices $w \geq v$. If a subtree with root $v$ contains only $v$ and an endpoint on scale $h_{v}+1$, it will be called 
a trivial subtree.

5) With each endpoint $v$ we associate one of the monomials with four or more Grassmann fields contributing to $\mathcal{R} \mathcal{V}^{(0)}\left(\Psi^{\left(\leq h_{v}-1\right)}\right)$, corresponding to the terms with $n \geq 2$ in the r.h.s. of (3.53) (with $\Psi^{(\leq 0)}$ replaced by $\Psi^{\left(\leq h_{v}-1\right)}$ ) and a set $\mathbf{x}_{v}$ of space-time points (the corresponding integration variables in the $\mathbf{x}$-space representation).

6) We introduce a field label $f$ to distinguish the field variables appearing in the terms associated with the endpoints as in item 3); the set of field labels associated with the endpoint $v$ will be called $I_{v}$; note that $\left|I_{v}\right|$ is the order of the monomial contributing to $\mathcal{V}^{(0)}\left(\Psi^{\left(\leq h_{v}-1\right)}\right)$ and associated to $v$. Analogously, if $v$ is not an endpoint, we shall call $I_{v}$ the set of field labels associated with the endpoints following the vertex $v ; \mathbf{x}(f), \varepsilon(f), \sigma(f), \rho(f)$ and $\omega(f)$ will denote the space-time point, the $\varepsilon$ index, the $\sigma$ index, the $\rho$ index and the $\omega$ index, respectively, of the Grassmann field variable with label $f$.

In terms of these trees, the effective potential $\mathcal{V}^{(h)}, h \leq-1$, can be written as

$$
\mathcal{V}^{(h)}\left(\Psi^{(\leq h)}\right)+\beta|\Lambda| \bar{e}_{k+1}=\sum_{n=1}^{\infty} \sum_{\tau \in \mathcal{T}_{h, n}} \mathcal{V}^{(h)}\left(\tau, \Psi^{(\leq h)}\right),
$$

where, if $v_{0}$ is the first vertex of $\tau$ and $\tau_{1}, \ldots, \tau_{s}\left(s=s_{v_{0}}\right)$ are the subtrees of $\tau$ with root $v_{0}$, $\mathcal{V}^{(h)}\left(\tau, \Psi^{(\leq h)}\right)$ is defined inductively as follows:

i) if $s>1$, then

$$
\mathcal{V}^{(h)}\left(\tau, \Psi^{(\leq h)}\right)=\frac{(-1)^{s+1}}{s !} \mathcal{E}_{h+1}^{T}\left[\overline{\mathcal{V}}^{(h+1)}\left(\tau_{1}, \Psi^{(\leq h+1)}\right) ; \ldots ; \overline{\mathcal{V}}^{(h+1)}\left(\tau_{s}, \Psi^{(\leq h+1)}\right)\right],
$$

where $\overline{\mathcal{V}}^{(h+1)}\left(\tau_{i}, \Psi^{(\leq h+1)}\right)$ is equal to $\mathcal{R} \mathcal{V}^{(h+1)}\left(\tau_{i}, \Psi^{(\leq h+1)}\right)$ if the subtree $\tau_{i}$ contains more than one end-point, or if it contains one end-point but it is not a trivial subtree; it is equal to $\mathcal{R} \mathcal{V}^{(0)}\left(\tau_{i}, \Psi^{(\leq h+1)}\right)$ if $\tau_{i}$ is a trivial subtree;

ii) if $s=1$, then $\mathcal{V}^{(h)}\left(\tau, \Psi^{(\leq h)}\right)$ is equal to $\mathcal{E}_{h+1}^{T}\left[\mathcal{R} \mathcal{V}^{(h+1)}\left(\tau_{1}, \Psi^{(\leq h+1)}\right)\right]$ if $\tau_{1}$ is not a trivial subtree; it is equal to $\mathcal{E}_{h+1}^{T}\left[\mathcal{R} \mathcal{V}^{(0)}\left(\Psi^{(\leq h+1)}\right)-\mathcal{R} \mathcal{V}^{(0)}\left(\Psi^{(\leq h)}\right)\right]$ if $\tau_{1}$ is a trivial subtree.

Using its inductive definition, the right hand side of (3.75) can be further expanded, and in order to describe the resulting expansion we need some more definitions.

We associate with any vertex $v$ of the tree a subset $P_{v}$ of $I_{v}$, the external fields of $v$. These subsets must satisfy various constraints. First of all, if $v$ is not an endpoint and $v_{1}, \ldots, v_{s_{v}}$ are the $s_{v} \geq 1$ vertices immediately following it, then $P_{v} \subseteq \cup_{i} P_{v_{i}}$; if $v$ is an endpoint, $P_{v}=I_{v}$. If $v$ is not an endpoint, we shall denote by $Q_{v_{i}}$ the intersection of $P_{v}$ and $P_{v_{i}}$; this definition implies that $P_{v}=\cup_{i} Q_{v_{i}}$. The union $\mathcal{I}_{v}$ of the subsets $P_{v_{i}} \backslash Q_{v_{i}}$ is, by definition, the set of the internal fields of $v$, and is non empty if $s_{v}>1$. Given $\tau \in \mathcal{T}_{h, n}$, there are many possible choices of the subsets $P_{v}, v \in \tau$, compatible with all the constraints. We shall denote $\mathcal{P}_{\tau}$ the family of all these choices and $\mathbf{P}$ the elements of $\mathcal{P}_{\tau}$.

With these definitions, we can rewrite $\mathcal{V}^{(h)}\left(\tau, \Psi^{(\leq h)}\right)$ in the r.h.s. of (3.75) as:

$$
\begin{aligned}
& \mathcal{V}^{(h)}\left(\tau, \Psi^{(\leq h)}\right)=\sum_{\mathbf{P} \in \mathcal{P}_{\tau}} \mathcal{V}^{(h)}(\tau, \mathbf{P}), \\
& \mathcal{V}^{(h)}(\tau, \mathbf{P})=\int d \mathbf{x}_{v_{0}} \widetilde{\Psi}^{(\leq h)}\left(P_{v_{0}}\right) K_{\tau, \mathbf{P}}^{(h+1)}\left(\mathbf{x}_{v_{0}}\right),
\end{aligned}
$$

where

$$
\widetilde{\Psi}^{(\leq h)}\left(P_{v}\right)=\prod_{f \in P_{v}} \Psi_{\mathbf{x}(f), \sigma(f), \rho(f), \omega(f)}^{(\leq h) \varepsilon(f)}
$$

and $K_{\tau, \mathbf{P}}^{(h+1)}\left(\mathbf{x}_{v_{0}}\right)$ is defined inductively by the equation, valid for any $v \in \tau$ which is not an endpoint,

$$
K_{\tau, \mathbf{P}}^{\left(h_{v}\right)}\left(\mathbf{x}_{v}\right)=\frac{1}{s_{v} !} \prod_{i=1}^{s_{v}}\left[K_{v_{i}}^{\left(h_{v}+1\right)}\left(\mathbf{x}_{v_{i}}\right)\right] \mathcal{E}_{h_{v}}^{T}\left[\widetilde{\Psi}^{\left(h_{v}\right)}\left(P_{v_{1}} \backslash Q_{v_{1}}\right), \ldots, \widetilde{\Psi}^{\left(h_{v}\right)}\left(P_{v_{s_{v}}} \backslash Q_{v_{s_{v}}}\right)\right]
$$

where $\widetilde{\Psi}^{\left(h_{v}\right)}\left(P_{v_{i}} \backslash Q_{v_{i}}\right)$ has a definition similar to (3.78). Moreover, if $v_{i}$ is an endpoint $K_{v_{i}}^{\left(h_{v}+1\right)}\left(\mathbf{x}_{v_{i}}\right)$ is equal to one of the kernels of the monomials contributing to $\mathcal{R V}^{(0)}\left(\Psi^{\left(\leq h_{v}\right)}\right)$, 
corresponding to the terms with $n \geq 2$ in the r.h.s. of (3.53) (with $\Psi^{(\leq 0)}$ replaced by $\Psi^{\left(\leq h_{v}\right)}$ ); if $v_{i}$ is not an endpoint, $K_{v_{i}}^{\left(h_{v}+1\right)}=K_{\tau_{i}, \mathbf{P}_{i}}^{\left(h_{v}+1\right)}$, where $\mathbf{P}_{i}=\left\{P_{w}, w \in \tau_{i}\right\}$.

(3.75) - 3.79 is not the final form of our expansion; we further decompose $\mathcal{V}^{(h)}(\tau, \mathbf{P})$, by using the following representation of the truncated expectation in the r.h.s. of (3.79). Let us put $s=s_{v}, P_{i} \equiv P_{v_{i}} \backslash Q_{v_{i}} ;$ moreover we order in an arbitrary way the sets $P_{i}^{ \pm} \equiv\left\{f \in P_{i}, \varepsilon(f)= \pm\right\}$, we call $f_{i j}^{ \pm}$their elements and we define $\mathbf{x}^{(i)}=\cup_{f \in P_{i}^{-}} \mathbf{x}(f), \mathbf{y}^{(i)}=\cup_{f \in P_{i}^{+}} \mathbf{x}(f), \mathbf{x}_{i j}=\mathbf{x}\left(f_{i j}^{-}\right)$, $\mathbf{y}_{i j}=\mathbf{x}\left(f_{i j}^{+}\right)$. Note that $\sum_{i=1}^{s}\left|P_{i}^{-}\right|=\sum_{i=1}^{s}\left|P_{i}^{+}\right| \equiv n$, otherwise the truncated expectation vanishes. A couple $l \equiv\left(f_{i j}^{-}, f_{i^{\prime} j^{\prime}}^{+}\right) \equiv\left(f_{l}^{-}, f_{l}^{+}\right)$will be called a line joining the fields with labels $f_{i j}^{-}, f_{i^{\prime} j^{\prime}}^{+}$, sector indices $\omega_{l}^{-}=\omega\left(f_{l}^{-}\right), \omega_{l}^{+}=\omega\left(f_{l}^{+}\right), \rho$-indices $\rho_{l}^{-}=\rho\left(f_{l}^{-}\right), \rho_{l}^{+}=\rho\left(f_{l}^{+}\right)$, and spin indices $\sigma_{l}^{-}=\sigma\left(f_{l}^{-}\right), \sigma_{l}^{+}=\sigma\left(f_{l}^{+}\right)$, connecting the points $\mathbf{x}_{l} \equiv \mathbf{x}_{i j}$ and $\mathbf{y}_{l} \equiv \mathbf{y}_{i^{\prime} j^{\prime}}$, the endpoints of $l$. Moreover, if $\omega_{l}^{-}=\omega_{l}^{+}$, we shall put $\omega_{l} \equiv \omega_{l}^{-}=\omega_{l}^{+}$. Then, we use the Brydges-BattleFederbush formula (e.g., see [13, 18]) saying that, up to a sign, if $s>1$,

$\mathcal{E}_{h}^{T}\left(\widetilde{\Psi}^{(h)}\left(P_{1}\right), \ldots, \widetilde{\Psi}^{(h)}\left(P_{s}\right)\right)=\sum_{T} \prod_{l \in T} \delta_{\omega_{l}^{-}, \omega_{l}^{+}} \delta_{\sigma_{l}^{-}, \sigma_{l}^{+}}\left[g_{\omega_{l}}^{(h)}\left(\mathbf{x}_{l}-\mathbf{y}_{l}\right)\right]_{\rho_{l}^{-}, \rho_{l}^{+}} \int d P_{T}(\mathbf{t}) \operatorname{det} G^{h, T}(\mathbf{t})$,

where $T$ is a set of lines forming an anchored tree graph between the clusters of points $\mathbf{x}^{(i)} \cup \mathbf{y}^{(i)}$, that is $T$ is a set of lines, which becomes a tree graph if one identifies all the points in the same cluster. Moreover $\mathbf{t}=\left\{t_{i i^{\prime}} \in[0,1], 1 \leq i, i^{\prime} \leq s\right\}, d P_{T}(\mathbf{t})$ is a probability measure with support on a set of $\mathbf{t}$ such that $t_{i i^{\prime}}=\mathbf{u}_{i} \cdot \mathbf{u}_{i^{\prime}}$ for some family of vectors $\mathbf{u}_{i} \in \mathbb{R}^{s}$ of unit norm. Finally $G^{h, T}(\mathbf{t})$ is a $(n-s+1) \times(n-s+1)$ matrix, whose elements are given by

$$
G_{i j, i^{\prime} j^{\prime}}^{h, T}=t_{i i^{\prime}} \delta_{\omega_{l}^{-}, \omega_{l}^{+}} \delta_{\sigma_{l}^{-}, \sigma_{l}^{+}}\left[g_{\omega_{l}}^{(h)}\left(\mathbf{x}_{i j}-\mathbf{y}_{i^{\prime} j^{\prime}}\right)\right]_{\rho_{l}^{-}, \rho_{l}^{+}}
$$

with $\left(f_{i j}^{-}, f_{i^{\prime} j^{\prime}}^{+}\right)$not belonging to $T$. In the following we shall use (3.78) even for $s=1$, when $T$ is empty, by interpreting the r.h.s. as equal to 1 , if $\left|P_{1}\right|=0$, otherwise as equal to $\operatorname{det} G^{h}=\mathcal{E}_{h}^{T}\left(\widetilde{\Psi}^{(h)}\left(P_{1}\right)\right)$.

Remark. It is crucial to note that $G^{h, T}$ is a Gram matrix, i.e., defining $\mathbf{e}_{+}=\mathbf{e}_{\uparrow}=(1,0)$ and $\mathbf{e}_{-}=\mathbf{e}_{\downarrow}=(0,1)$, the matrix elements in (3.81) can be written in terms of scalar products:

$$
\begin{aligned}
& t_{i i^{\prime}} \delta_{\omega_{l}^{-}, \omega_{l}^{+}} \delta_{\sigma_{l}^{-}, \sigma_{l}^{+}}\left[g_{\omega_{l}}^{(h)}\left(\mathbf{x}_{i j}-\mathbf{y}_{i^{\prime} j^{\prime}}\right)\right]_{\rho_{l}^{-}, \rho_{l}^{+}}= \\
& \quad=\left(\mathbf{u}_{i} \otimes \mathbf{e}_{\omega_{l}^{-}} \otimes \mathbf{e}_{\sigma_{l}^{-}} \otimes A\left(\mathbf{x}_{i j}-\cdot\right), \mathbf{u}_{i^{\prime}} \otimes \mathbf{e}_{\omega_{l}^{+}} \otimes \mathbf{e}_{\sigma_{l}^{+}} \otimes B\left(\mathbf{x}_{i^{\prime} j^{\prime}}-\cdot\right)\right) \equiv\left(\mathbf{f}_{\alpha}, \mathbf{g}_{\beta}\right),
\end{aligned}
$$

where

$$
\begin{aligned}
& A(\mathbf{x})=\frac{1}{\beta|\Lambda|} \sum_{\mathbf{k}^{\prime} \in \mathcal{D}_{\beta, L}^{\omega}} e^{-i \mathbf{k}^{\prime} \mathbf{x}} \sqrt{f_{h}\left(\mathbf{k}^{\prime}\right)} \mathbb{1} \\
& B(\mathbf{x})=\frac{1}{\beta|\Lambda|} \sum_{\mathbf{k}^{\prime} \in \mathcal{D}_{\beta, L}^{\omega}} e^{-i \mathbf{k}^{\prime} \mathbf{x}} \sqrt{f_{h}\left(\mathbf{k}^{\prime}\right)}\left[\bar{A}_{h-1, \omega}\left(\mathbf{k}^{\prime}\right)\right]^{-1} .
\end{aligned}
$$

The symbol $(\cdot, \cdot)$ denotes the inner product, i.e.,

$$
\begin{aligned}
& \left(\mathbf{u}_{i} \otimes \mathbf{e}_{\omega} \otimes \mathbf{e}_{\sigma} \otimes A(\mathbf{x}-\cdot), \mathbf{u}_{i^{\prime}} \otimes \mathbf{e}_{\omega^{\prime}} \otimes \mathbf{e}_{\sigma^{\prime}} \otimes B\left(\mathbf{x}^{\prime}-\cdot\right)\right)= \\
& =\left(\mathbf{u}_{i} \cdot \mathbf{u}_{i^{\prime}}\right)\left(\mathbf{e}_{\omega} \cdot \mathbf{e}_{\omega^{\prime}}\right)\left(\mathbf{e}_{\sigma} \cdot \mathbf{e}_{\sigma^{\prime}}\right) \cdot \int d \mathbf{z} A^{*}(\mathbf{x}-\mathbf{z}) B\left(\mathbf{x}^{\prime}-\mathbf{z}\right),
\end{aligned}
$$

and the vectors $\mathbf{f}_{\alpha}, \mathbf{g}_{\beta}$ with $\alpha, \beta=1, \ldots, n-s+1$ are implicitely defined by (3.82). The usefulness of the representation (3.82) is that, by the Gram-Hadamard inequality (see, e.g., [13] $),\left|\operatorname{det}\left(\mathbf{f}_{\alpha}, \mathbf{g}_{\beta}\right)\right| \leq \prod_{\alpha}\left\|f_{\alpha}\right\|\left\|g_{\alpha}\right\|$. In our case, $\left\|\mathbf{f}_{\alpha}\right\| \leq C \gamma^{3 h / 2}$ and $\left\|\mathbf{g}_{\alpha}\right\| \leq C \gamma^{h / 2}$. Therefore, $\left\|f_{\alpha}\right\|\left\|g_{\alpha}\right\| \leq C \gamma^{2 h}$, uniformly in $\alpha$, so that the Gram determinant can be bounded by $C^{n-s+1} \gamma^{2 h(n-s+1)}$. 
If we apply the expansion (3.80) in each vertex of $\tau$ different from the endpoints, we get an expression of the form

$$
\mathcal{V}^{(h)}(\tau, \mathbf{P})=\sum_{T \in \mathbf{T}} \int d \mathbf{x}_{v_{0}} \widetilde{\Psi}^{(\leq h)}\left(P_{v_{0}}\right) W_{\tau, \mathbf{P}, T}^{(h)}\left(\mathbf{x}_{v_{0}}\right) \equiv \sum_{T \in \mathbf{T}} \mathcal{V}^{(h)}(\tau, \mathbf{P}, T),
$$

where $\mathbf{T}$ is a special family of graphs on the set of points $\mathbf{x}_{v_{0}}$, obtained by putting together an anchored tree graph $T_{v}$ for each non trivial vertex $v$. Note that any graph $T \in \mathbf{T}$ becomes a tree graph on $\mathbf{x}_{v_{0}}$, if one identifies all the points in the sets $\mathbf{x}_{v}$, with $v$ an endpoint. Given $\tau \in \mathcal{T}_{h, n}$ and the labels $\mathbf{P}, T$, calling $v_{i}^{*}, \ldots, v_{n}^{*}$ the endpoints of $\tau$ and putting $h_{i}=h_{v_{i}^{*}}$, the explicit representation of $W_{\tau, \mathbf{P}, T}^{(h)}\left(\mathbf{x}_{v_{0}}\right)$ in (3.85) is

$$
\begin{aligned}
& W_{\tau, \mathbf{P}, T}\left(\mathbf{x}_{v_{0}}\right)=\left[\prod_{i=1}^{n} K_{v_{i}^{*}}^{\left(h_{i}\right)}\left(\mathbf{x}_{v_{i}^{*}}\right)\right] . \\
& \left\{\prod_{\substack{v \\
\text { not e.p. }}} \frac{1}{s_{v} !} \int d P_{T_{v}}\left(\mathbf{t}_{v}\right) \operatorname{det} G^{h_{v}, T_{v}}\left(\mathbf{t}_{v}\right)\left[\prod_{l \in T_{v}} \delta_{\omega_{l}^{-}, \omega_{l}^{+}} \delta_{\sigma_{l}^{-}, \sigma_{l}^{+}}\left[g_{\omega_{l}}^{(h)}\left(\mathbf{x}_{l}-\mathbf{y}_{l}\right)\right]_{\rho_{l}^{-}, \rho_{l}^{+}}\right]\right\},
\end{aligned}
$$

Analyticity of the effective potentials. The tree expansion described above allows us to express the effective potential $\mathcal{V}^{(h)}$ in terms of the running coupling constants $\zeta_{h}, c_{h}$ and of the renormalization functions $\sigma_{k}(\mathbf{k}), t_{k, \omega}(\mathbf{k})$.

The next goal will be the proof of the following result.

TheOREm 2. There exists a constants $U_{0}>0$ such that, if $|U| \leq U_{0}$, then the kernels $W_{2 l, \underline{\rho}, \underline{\omega}}^{(h)}\left(\mathbf{x}_{1}, \ldots, \mathbf{x}_{2 l}\right)$ in (3.58), $h \leq-1$, are analytic functions of $U$, satisfying, for any $0 \leq \bar{\theta}<1$ and a suitable constant $C>0$, the following estimates:

$$
\frac{1}{\beta|\Lambda|} \int d \mathbf{x}_{1} \cdots d \mathbf{x}_{2 l}\left|W_{2 l, \underline{\rho}, \underline{\omega}}^{(h)}\left(\mathbf{x}_{1}, \ldots, \mathbf{x}_{2 l}\right)\right| \leq \gamma^{h(3-2 l+\theta)}(C|U|)^{\max (1, l-1)} .
$$

Moreover, the constants $e_{h}$ and $\bar{e}_{h}$ defined by (3.68) and (3.73) are also analytic functions of $U$ in the domain $|U| \leq U_{0}$, and there they satisfy the estimate $\left|\bar{e}_{h}\right|+\left|e_{h}\right| \leq C U_{0} \gamma^{h(3+\theta)}$.

Remark. The above result immediately implies the analyticity of the specific free energy $f_{\beta}(U)$ and of its zero temperature limit $e(U)$, i.e., of the specific ground state energy. In fact, by construction, $f_{\beta}(U)=F_{0}+\sum_{h=h_{\beta}}^{0}\left(e_{h}+\bar{e}_{h}\right)$, with $F_{0}$ an analytic function of $U$, see the discussion after (3.36) and in Appendix B. Therefore, Theorem 2 implies the part of the statement of Theorem 1 concerning the free energy and the ground state energy. For the proof of analyticity of the Schwinger functions, see next Section.

Proof of Theorem 2. Let us preliminarily assume that, for $h \leq-1$, and for suitable constants $c, c_{n}$, the corrections $z_{h}, \delta_{h}, \sigma_{h}\left(\mathbf{k}^{\prime}\right)$ and $\tau_{h}\left(\mathbf{k}^{\prime}\right)$ defined in (3.62) and (3.64), satisfy the following estimates:

$$
\begin{gathered}
\max \left\{\left|z_{h}\right|,\left|\delta_{h}\right|\right\} \leq c|U| \gamma^{\theta h}, \\
\sup _{\left|\mathbf{k}^{\prime}\right| \text { s.t. } \chi_{h}\left(\mathbf{k}^{\prime}\right) \neq 0}\left\{|| \partial_{\mathbf{k}^{\prime}}^{n} \sigma_{h}\left(\mathbf{k}^{\prime}\right) \|,|| \partial_{\mathbf{k}^{\prime}}^{n} \tau_{h, \omega}\left(\mathbf{k}^{\prime}\right)||\right\} \leq c_{n}|U| \gamma^{(1+\theta-n) h} .
\end{gathered}
$$

Using (3.88) we inductively see that the running coupling functions $\zeta_{h}, c_{h}, s_{h}\left(\mathbf{k}^{\prime}\right)$ and $t_{h}\left(\mathbf{k}^{\prime}\right)$ satisfy similar estimates:

$$
\begin{gathered}
\max \left\{\left|\zeta_{h}-1\right|,\left|c_{h}-3 / 2\right|\right\} \leq c|U| \\
\sup _{\left|\mathbf{k}^{\prime}\right| \text { s.t. } \chi_{h}\left(\mathbf{k}^{\prime}\right) \neq 0}\left\{|| \partial_{\mathbf{k}^{\prime}}^{n} s_{h}\left(\mathbf{k}^{\prime}\right)||,|| \partial_{\mathbf{k}^{\prime}}^{n} t_{h, \omega}\left(\mathbf{k}^{\prime}\right)||\right\} \leq c_{n}|U| \gamma^{(1+\theta-n) h}
\end{gathered}
$$

Now, using the definition of $g_{\omega}^{(h)}$, see (3.72) and (3.66), we get, after integration by parts, for any $N \geq 0$,

$$
\left\|\left[\partial_{x_{0}}^{n_{0}} \partial_{x_{1}}^{n_{1}} \partial_{x_{2}}^{n_{2}} g_{\omega}^{(h)}\left(\mathbf{x}_{1}, \mathbf{x}_{2}\right)\right]_{\rho, \rho^{\prime}}\right\| \leq C_{N, n} \frac{\gamma^{(2+n) h}}{1+\left(\gamma^{h}\left|\mathbf{x}_{1}-\mathbf{x}_{2}\right|\right)^{N}}
$$


where $n=n_{0}+n_{1}+n_{2} \geq 0$ and $C_{N, n}$ is a suitable constant.

Using the tree expansion described above and, in particular, Eqs.(3.75), (3.77), (3.85) and (3.86), we find that the l.h.s. of (3.87) can be bounded from above by

$$
\begin{aligned}
\sum_{n \geq 1} \sum_{\tau \in \mathcal{T}_{h, n}} \sum_{\substack{\mathbf{P} \in \mathcal{P}_{\tau} \\
\left|P_{v_{0}}\right|=2 l}} \sum_{T \in \mathbf{T}} \int \prod_{l \in T^{*}} d\left(\mathbf{x}_{l}-\mathbf{y}_{l}\right)\left[\prod_{i=1}^{n}\left|K_{v_{i}^{*}}^{\left(h_{i}\right)}\left(\mathbf{x}_{v_{i}^{*}}\right)\right|\right] \\
\cdot\left[\prod_{v \text { not e.p. }} \frac{1}{s_{v} !} \max _{\mathbf{t}_{v}}\left|\operatorname{det} G^{h_{v}, T_{v}}\left(\mathbf{t}_{v}\right)\right| \prod_{l \in T_{v}}\left\|g_{\omega_{l}}^{(h)}\left(\mathbf{x}_{l}-\mathbf{y}_{l}\right)\right\|\right]
\end{aligned}
$$

where $\|\cdot\|$ is the spectral norm and where $T^{*}$ is a tree graph obtained from $T=\cup_{v} T_{v}$, by adding in a suitable (obvious) way, for each endpoint $v_{i}^{*}, i=1, \ldots, n$, one or more lines connecting the space-time points belonging to $\mathbf{x}_{v_{i}^{*}}$.

A standard application of Gram-Hadamard inequality, combined with the dimensional bound on $g_{\omega}^{(h)}(\mathbf{x})$ given by (3.90), see the remark after (3.81), implies that

$$
\left|\operatorname{det} G^{h_{v}, T_{v}}\left(\mathbf{t}_{v}\right)\right| \leq c^{\sum_{i=1}^{s_{v}}\left|P_{v_{i}}\right|-\left|P_{v}\right|-2\left(s_{v}-1\right)} \cdot \gamma^{h_{v}\left(\sum_{i=1}^{s_{v}}\left|P_{v_{i}}\right|-\left|P_{v}\right|-2\left(s_{v}-1\right)\right)} .
$$

By the decay properties of $g_{\omega}^{(h)}(\mathbf{x})$ given by (3.90), it also follows that

$$
\prod_{v \text { not e.p. }} \frac{1}{s_{v} !} \int \prod_{l \in T_{v}} d\left(\mathbf{x}_{l}-\mathbf{y}_{l}\right)\left\|g_{\omega_{l}}^{\left(h_{v}\right)}\left(\mathbf{x}_{l}-\mathbf{y}_{l}\right)\right\| \leq c^{n} \prod_{v \text { not e.p. }} \frac{1}{s_{v} !} \gamma^{-h_{v}\left(s_{v}-1\right)} .
$$

The bound (3.37) on the kernels produced by the ultraviolet integration implies that

$$
\int \prod_{l \in T^{*} \backslash \cup_{v} T_{v}} d\left(\mathbf{x}_{l}-\mathbf{y}_{l}\right) \prod_{i=1}^{n}\left|K_{v_{i}^{*}}^{\left(h_{i}\right)}\left(\mathbf{x}_{v_{i}^{*}}\right)\right| \leq \prod_{i=1}^{n} C^{p_{i}}|U|^{\frac{p_{i}}{2}-1},
$$

where $p_{i}=\left|P_{v_{i}^{*}}\right|$. Combining the previous bounds, we find that (3.91) can be bounded above by

$$
\sum_{n \geq 1} \sum_{\tau \in \mathcal{T}_{h, n}} \sum_{\substack{\mathbf{P} \in \mathcal{P}_{\tau} \\\left|P_{v_{0}}\right|=2 l}} \sum_{T \in \mathbf{T}} C^{n}\left[\prod_{v} \frac{1}{\text { not e.p. }} \frac{1}{s_{v} !} \gamma^{h_{v}}\left(\sum_{i=1}^{s_{v}}\left|P_{v_{i}}\right|-\left|P_{v}\right|-3\left(s_{v}-1\right)\right)\right]\left[\prod_{i=1}^{n} C^{p_{i}}|U|^{\frac{p_{i}}{2}-1}\right]
$$

Let us define $n(v)=\sum_{i: v_{i}^{*}>v} 1$ as the number of endpoints following $v$ on $\tau$ and $v^{\prime}$ as the vertex immediately preceding $v$ on $\tau$. Recalling that $\left|I_{v}\right|$ is the number of field labels associated to the endpoints following $v$ on $\tau$ (note that $\left|I_{v}\right| \geq 4 n(v)$ ) and using that

$$
\begin{aligned}
& \sum_{v \text { not e.p. }}\left[\left(\sum_{i=1}^{s_{v}}\left|P_{v_{i}}\right|\right)-\left|P_{v}\right|\right]=\left|I_{v_{0}}\right|-\left|P_{v_{0}}\right|, \\
& \sum_{v \text { not e.p. }}\left(s_{v}-1\right)=n-1, \\
& \sum_{v \text { not e.p. }}\left(h_{v}-h\right)\left[\left(\sum_{i=1}^{s_{v}}\left|P_{v_{i}}\right|\right)-\left|P_{v}\right|\right]=\sum_{v \text { not e.p. }}\left(h_{v}-h_{v^{\prime}}\right)\left(\left|I_{v}\right|-\left|P_{v}\right|\right), \\
& \sum_{v \text { not e.p. }}\left(h_{v}-h\right)\left(s_{v}-1\right)=\sum_{v \text { not e.p. }}\left(h_{v}-h_{v^{\prime}}\right)(n(v)-1),
\end{aligned}
$$

we find that (3.95) can be bounded above by

$$
\begin{aligned}
\sum_{n \geq 1} \sum_{\tau \in \mathcal{T}_{h, n}} \sum_{\substack{\mathbf{P} \in \mathcal{P}_{\tau} \\
\left|P_{v_{0}}\right|=2 l}} \sum_{T \in \mathbf{T}} C^{n} \gamma^{h\left(3-\left|P_{v_{0}}\right|+\left|I_{v_{0}}\right|-3 n\right)} . \\
\cdot\left[\prod_{v} \frac{1}{\text { not e.p. }} \frac{1}{s_{v} !} \gamma^{\left(h_{v}-h_{v^{\prime}}\right)\left(3-\left|P_{v}\right|+\left|I_{v}\right|-3 n(v)\right)}\right]\left[\prod_{i=1}^{n} C^{\left.p_{i}|U|^{\frac{p_{i}}{2}-1}\right] .}\right.
\end{aligned}
$$


Finally, let $\bar{n}(v)$ be the number of endpoints following $v$ but not following any of the vertices $w>v$ and let $\bar{p}(v)$ be the number of field labels associated to endpoints following $v$ but not following any of the vertices $w>v$. Using the identities

$$
\begin{aligned}
& \gamma^{h n} \prod_{v \text { not e.p. }} \gamma^{\left(h_{v}-h_{v^{\prime}}\right) n(v)}=\prod_{v \text { not e.p. }} \gamma^{h_{v} \bar{n}(v)}, \\
& \gamma^{h\left|I_{v_{0}}\right|} \prod_{v \text { not e.p. }} \gamma^{\left(h_{v}-h_{v^{\prime}}\right)\left|I_{v}\right|}=\prod_{v \text { not e.p. }} \gamma^{h_{v} \bar{p}(v)}
\end{aligned}
$$

we obtain

$$
\begin{aligned}
& \frac{1}{\beta|\Lambda|} \int d \mathbf{x}_{1} \cdots d \mathbf{x}_{2 l}\left|W_{2 l, \underline{\rho}, \underline{\omega}}^{(h)}\left(\mathbf{x}_{1}, \ldots, \mathbf{x}_{2 l}\right)\right| \leq \sum_{n \geq 1} \sum_{\tau \in \mathcal{T}_{h, n}} \sum_{\substack{\mathbf{P} \in \mathcal{P}_{\tau} \\
\left|P_{v_{0}}\right|=2 l}} \sum_{T \in \mathbf{T}} C^{n} \gamma^{h\left(3-\left|P_{v_{0}}\right|\right) .} \\
& \cdot\left[\prod_{v \text { not e.p. }} \frac{1}{s_{v} !} \gamma^{-\left(h_{v}-h_{v^{\prime}}\right)\left(\left|P_{v}\right|-3\right)}\right]\left[\prod_{v \text { not e.p. }} \gamma^{h_{v}(\bar{p}(v)-3 \bar{n}(v))}\right]\left[\prod_{i=1}^{n} C^{p_{i}} \mid U^{\frac{p_{i}}{2}-1}\right] .
\end{aligned}
$$

Note that, if $v$ is not an endpoint, $\left|P_{v}\right|-3 \geq 1$ by the definition of $\mathcal{R}$. Moreover $\bar{p}(v)-3 \bar{n}(v) \geq 0$ and $\sum_{v \text { not e.p. }}(\bar{p}(v)-3 \bar{n}(v)) \geq n$; in particular, this means that there exists at least one vertex $v^{*}$ that is not an endpoint, such that $\bar{p}\left(v^{*}\right)-3 \bar{n}\left(v^{*}\right) \geq 1$. Therefore, we get

$$
\prod_{v \text { not e.p. }} \gamma^{h_{v}(\bar{p}(v)-3 \bar{n}(v))} \leq \gamma^{h_{*}}
$$

with $h_{*}$ the highest scale label of the tree. Now, note that the number of terms in $\sum_{T \in \mathbf{T}}$ can be bounded by $C^{n} \prod_{v}$ not e.p. $s_{v}$ !. Using also that $\left|P_{v}\right|-3 \geq 1$ and $\left|P_{v}\right|-3 \geq\left|P_{v}\right| / 4$, we find that the 1.h.s. of (3.99) can be bounded as

$$
\begin{aligned}
& \frac{1}{\beta|\Lambda|} \int d \mathbf{x}_{1} \cdots d \mathbf{x}_{2 l}\left|W_{2 l, \underline{\rho}, \underline{\omega}}^{(h)}\left(\mathbf{x}_{1}, \ldots, \mathbf{x}_{2 l}\right)\right| \leq \gamma^{h\left(3-\left|P_{v_{0}}\right|\right)} \sum_{n \geq 1} C^{n} \sum_{\tau \in \mathcal{T}_{h, n}} \gamma^{h_{*}} . \\
& \cdot\left(\prod_{v \text { not e.p. }} \gamma^{-\theta\left(h_{v}-h_{v^{\prime}}\right)} \gamma^{-(1-\theta)\left(h_{v}-h_{v^{\prime}}\right) / 2}\right) \sum_{\substack{\mathbf{P} \in \mathcal{P}_{\tau} \\
\left|P_{v_{0}}\right|=2 l}}\left(\prod_{v \text { not e.p. }} \gamma^{-(1-\theta)\left|P_{v}\right| / 8}\right) \prod_{i=1}^{n} C^{p_{i}|U|^{\frac{p_{i}}{2}-1}} .
\end{aligned}
$$

Now, the sum over $\mathbf{P}$ can be bounded using the following combinatorial inequality (see for instance $\S \mathrm{A} 6.1$ of [13] $)$ : let $\left\{p_{v}, v \in \tau\right\}$, with $\tau \in \mathcal{T}_{h, n}$, a set of integers such that $p_{v} \leq \sum_{i=1}^{s_{v}} p_{v_{i}}$ for all $v \in \tau$ which are not endpoints; then, if $\alpha>0$,

$$
\prod_{\text {v not e.p. }} \sum_{p_{v}} \gamma^{-\alpha p_{v}} \leq C_{\alpha}^{n}
$$

This implies that

$$
\sum_{\substack{\mathbf{P} \in \mathcal{P}_{\tau} \\\left|P_{v_{0}}\right|=2 l}}\left(\prod_{v \text { not e.p. }} \gamma^{-(1-\theta)\left|P_{v}\right| / 8}\right) \prod_{i=1}^{n} C^{p_{i}}|U|^{\frac{p_{i}}{2}-1} \leq C_{\theta}^{n}|U|^{n}
$$

Finally, using that $\gamma^{h_{*}} \prod_{v \text { not e.p. }} \gamma^{-\theta\left(h_{v}-h_{v^{\prime}}\right)} \leq \gamma^{\theta h}$, and that, for $0<\theta<1$,

$$
\sum_{\tau \in \mathcal{T}_{h, n}} \prod_{v} \gamma^{-(1-\theta)\left(h_{v}-h_{v^{\prime}}\right) / 2} \leq C^{n}
$$

as it follows by the fact that the number of non trivial vertices in $\tau$ is smaller than $n-1$ and that the number of trees in $\mathcal{T}_{h, n}$ is bounded by const $^{n}$, and collecting all the previous bounds, we obtain

$$
\frac{1}{\beta|\Lambda|} \int d \mathbf{x}_{1} \cdots d \mathbf{x}_{2 l}\left|W_{2 l, \underline{\rho}, \underline{\omega}}^{(h)}\left(\mathbf{x}_{1}, \ldots, \mathbf{x}_{2 l}\right)\right| \leq \gamma^{h\left(3-\left|P_{v_{0}}\right|+\theta\right)} \sum_{n \geq 1} C^{n}|U|^{n}
$$


which is the desired result.

It remains to prove the assumption (3.88). We proceed by induction. The assumption is valid for $h=0$, as it follows by (3.37) and by the discussion in Appendix B. Now, assume that (3.88) is valid for all $h \geq k+1$, and let us prove it for $k-1$. The functions $-i z_{k} k_{0}+\sigma_{k}\left(\mathbf{k}^{\prime}\right)$ and $\delta_{k}\left(i k_{1}^{\prime}-\omega k_{2}^{\prime}\right)+\tau_{k, \omega}\left(\mathbf{k}^{\prime}\right)$ admit a representation in terms of $W_{2, \underline{\rho},(\omega, \omega)}^{(k)}(\mathbf{x}, \mathbf{y})$. In particular,

$$
\max \left\{\left|z_{k}\right|,\left|\delta_{k}\right|\right\} \leq \frac{1}{\beta|\Lambda|} \int d \mathbf{x}_{1} d \mathbf{x}_{2}|\mathbf{x}-\mathbf{y}|\left|W_{2, \underline{\rho},(\omega, \omega)}^{(k)}(\mathbf{x}, \mathbf{y})\right|
$$

and

$$
\sup _{\left|\mathbf{k}^{\prime}\right| \text { s.t. } \chi_{k}\left(\mathbf{k}^{\prime}\right) \neq 0}\left\{\left\|\partial_{\mathbf{k}^{\prime}}^{n} \sigma_{k}\left(\mathbf{k}^{\prime}\right)\right\|,\left\|\partial_{\mathbf{k}^{\prime}}^{n} \tau_{k, \omega}\left(\mathbf{k}^{\prime}\right)\right\|\right\} \leq C \gamma^{2 k} \frac{1}{\beta|\Lambda|} \int d \mathbf{x}_{1} d \mathbf{x}_{2}|\mathbf{x}-\mathbf{y}|^{n+2}\left|W_{2, \underline{\rho},(\omega, \omega)}^{(k)}(\mathbf{x}, \mathbf{y})\right| .
$$

The same proof leading to (3.102) shows that the r.h.s. of (3.103) can be bounded by the r.h.s. of (3.102) times $\gamma^{-k}$ (that is the dimensional estimate for $|\mathbf{x}-\mathbf{y}|$ ), and that the r.h.s. of (3.103) can be bounded by the r.h.s. of (3.102) times $\gamma^{2 k} \gamma^{-(n+2) k}$ (where $\gamma^{-k(n+2)}$ is the dimensional estimate for $\left.|\mathbf{x}-\mathbf{y}|^{n+2}\right)$. This concludes the proof of Theorem 2 .

\section{The two point Schwinger function}

In this section we describe how to modify the expansion for the free energy described in previous sections in order to compute the Schwinger functions at distinct space-time points. For simplicity, we shall restrict our attention to the case of the two point Schwinger function. The general case can be worked out along the same lines.

The Schwinger functions can be derived from the generating function defined as

$$
\mathcal{W}(\phi)=\log \int P(d \Psi) e^{-\mathcal{V}(\psi)+\int d \mathbf{x}\left[\phi_{\mathbf{x}, \sigma, \rho}^{+} \Psi_{\mathbf{x}, \sigma, \rho}^{-}+\Psi_{\mathbf{x}, \sigma, \rho}^{+} \phi_{\mathbf{x}, \sigma, \rho}^{-}\right]}
$$

where summation over repeated indices is understood and the variables $\phi_{\mathbf{x}, \sigma, \rho}^{\varepsilon}$ are Grassmann variables, anticommuting among themselves and with the variables $\Psi_{\mathbf{x}, \sigma, \rho}^{\varepsilon}$. The two-point Schwinger function $S(\mathbf{x}-\mathbf{y})_{\rho, \rho^{\prime}} \stackrel{\text { def }}{=} S_{2}\left(\mathbf{x}, \sigma,-, \rho ; \mathbf{y}, \sigma,+, \rho^{\prime}\right)$ is given by

$$
S(\mathbf{x}-\mathbf{y})_{\rho, \rho^{\prime}}=\left.\frac{\partial^{2}}{\partial \phi_{\mathbf{x}, \sigma, \rho}^{+} \partial \phi_{\mathbf{y}, \sigma, \rho^{\prime}}^{-}} \mathcal{W}(\phi)\right|_{\phi=0} .
$$

We start by studying the generating function and, in analogy with the procedure described before, we begin by decomposing the field $\Psi$ in an ultraviolet and an infrared component: $\Psi=\Psi^{(u . v .)}+\Psi^{(i . r .)}$, with $\Psi_{\mathbf{x}, \sigma, \rho}^{(i . r .) \pm}=\sum_{\omega= \pm} e^{i \vec{p}_{F}^{\omega} \vec{x}} \Psi_{\mathbf{x}, \sigma, \rho, \omega}^{(\leq 0) \pm}$. After the integration of the $\Psi^{(u . v .)}$ variables, and after rewriting $\phi_{\mathbf{x}, \sigma, \rho}^{ \pm}=\sum_{\omega= \pm} e^{i \vec{p}_{F}^{\omega} \vec{x}_{\mathbf{x}, \sigma, \rho}^{ \pm}}$, we get:

$$
\begin{aligned}
& e^{\mathcal{W}(\phi)}=e^{-\beta|\Lambda| F_{0}+S^{(\geq 0)}(\phi)} \int P_{\chi_{0}, A_{0}}\left(d \Psi^{(\leq 0)}\right) . \\
& \cdot e^{-\mathcal{V}^{(0)}\left(\psi^{(\leq 0)}\right)-B^{(0)}\left(\Psi^{(\leq 0)}, \phi\right)+\int d \mathbf{x}\left[\phi_{\mathbf{x}, \sigma, \rho, \omega}^{+} \Psi_{\mathbf{x}, \sigma, \rho, \omega}^{(\leq 0)-}+\Psi_{\mathbf{x}, \sigma, \rho, \omega}^{(\leq 0)+} \phi_{\mathbf{x}, \sigma, \rho, \omega}^{-}\right]}
\end{aligned}
$$

where $S^{(\geq 0)}(\phi)$ (chosen in such a way that $S^{(\geq 0)}(0)=0$ ) collects the terms depending on $\phi$ but not on $\Psi^{(\leq 0)}$ and $B^{(0)}\left(\Psi^{(\leq 0)}, \phi\right)$ the terms depending both on $\phi$ and $\Psi^{(\leq 0)}$ generated by the ultraviolet integration.

Proceeding as in Section IIIC, we inductively show (see below for details) that, if $h \leq 0$, $e^{\mathcal{W}(\phi)}$ can be rewritten as:

$$
\begin{aligned}
& e^{\mathcal{W}(\phi)}=e^{-\beta|\Lambda| F_{h}+S^{(\geq h)}(\phi)} \int P_{\chi_{h}, A_{h}}\left(d \Psi^{(\leq h)}\right) . \\
& \left.\cdot e^{-\mathcal{V}^{(h)}\left(\Psi^{(\leq h)}\right)-B^{(h)}\left(\Psi^{(\leq h)}, \phi\right)+\int d \mathbf{k}^{\prime}\left[\hat{\phi}_{\mathbf{k}^{\prime}, \sigma, \rho, \omega}^{+} \hat{Q}_{\mathbf{k}^{\prime}, \omega, \rho, \rho^{\prime}}^{(h+1)} \hat{\Psi}_{\mathbf{k}^{\prime}, \sigma, \rho^{\prime}, \omega}^{(\leq h)-}+\hat{\Psi}_{\mathbf{k}^{\prime}, \sigma, \rho, \omega}^{(\leq h)+} \hat{Q}_{\mathbf{k}^{\prime}, \omega, \rho, \rho^{\prime}}^{(h+1)} \hat{\phi}_{\mathbf{k}^{\prime}, \sigma, \rho^{\prime}, \omega}^{-}\right.}\right]
\end{aligned}
$$


where: $\int d \mathbf{k}^{\prime}$ must be interpreted as equal to $(\beta|\Lambda|)^{-1} \sum_{\mathbf{k} \in \mathcal{D}_{\beta, L}^{\omega}} ; B^{(h)}\left(\Psi^{(\leq h)}, \phi\right)$ can be written as $B_{\phi}^{(h)}\left(\Psi^{(\leq h)}\right)+W_{R}^{(h)}$, with $W_{R}^{(h)}$ containing the terms of third or higher order in $\phi$ and $B_{\phi}^{(h)}\left(\Psi^{(\leq h)}\right)$ of the form

$$
\begin{aligned}
& \int d \mathbf{x}\left[\phi_{\cdot, \sigma, \rho_{1}, \omega}^{+} * G_{\omega, \rho_{1}, \rho_{2}}^{(h+1)} * \frac{\partial \mathcal{V}^{(h)}\left(\Psi^{(\leq h)}\right)}{\partial \Psi_{\left(, \sigma, \rho_{2}, \omega\right.}^{(\leq h)+}}+\frac{\partial \mathcal{V}^{(h)}\left(\Psi^{(\leq h)}\right)}{\partial \Psi_{\cdot, \sigma, \rho_{1}, \omega}^{(\leq h)-}} * G_{\omega, \rho_{1}, \rho_{2}}^{(h+1)} * \phi_{\cdot, \sigma, \rho_{2}, \omega}^{-}+\right. \\
& \left.+\phi_{\cdot, \sigma_{1}, \rho_{1}, \omega_{1}}^{+} * G_{\omega_{1}, \rho_{1}, \rho_{2}}^{(h+1)} * \frac{\partial^{2}}{\partial \Psi_{\cdot, \sigma_{1}, \rho_{2}, \omega_{1}}^{(\leq h)+} \partial \Psi_{\cdot, \sigma_{2}, \rho_{3}, \omega_{2}}^{(\leq h)-}} \mathcal{R V}^{(h)}\left(\Psi^{(\leq h)}\right) * G_{\omega_{2}, \rho_{3}, \rho_{4}}^{(h+1)} * \phi_{\cdot, \sigma_{2}, \rho_{4}, \omega_{2}}^{-}\right]
\end{aligned}
$$

with

$$
\hat{G}_{\omega, \rho, \rho^{\prime}}^{(h+1)}\left(\mathbf{k}^{\prime}\right)=\sum_{k=h+1}^{1} \hat{g}_{\omega, \rho, \rho^{\prime \prime}}^{(k)}\left(\mathbf{k}^{\prime}\right) \hat{Q}_{\mathbf{k}^{\prime}, \omega, \rho^{\prime \prime}, \rho^{\prime}}^{(k)}
$$

and $\hat{Q}_{\mathbf{k}^{\prime}, \omega, \rho, \rho^{\prime}}^{(h)}$ defined inductively by the relations

$$
\hat{Q}_{\mathbf{k}^{\prime}, \omega, \rho, \rho^{\prime}}^{(h)}=\hat{Q}_{\mathbf{k}^{\prime}, \omega, \rho, \rho^{\prime}}^{(h+1)}-W_{2, \rho, \rho^{\prime \prime},(\omega, \omega)}^{(h)}\left(\mathbf{k}^{\prime}\right) \hat{G}_{\omega, \rho^{\prime \prime}, \rho^{\prime}}^{(h+1)}\left(\mathbf{k}^{\prime}\right), \quad Q_{\mathbf{k}^{\prime}, \omega, \rho, \rho^{\prime}}^{(1)} \equiv \delta_{\rho, \rho^{\prime}},
$$

where $W_{2, \underline{\rho}, \underline{\omega}}^{(h)}$ is the kernel of $\mathcal{L} \mathcal{V}^{(h)}$, as defined in (3.61). In (3.110), $\hat{g}_{\omega}^{(1)}$ is defined as

$$
\hat{g}_{\omega}^{(1)}\left(\mathbf{k}^{\prime}\right)=\hat{g}^{(u . v .)}\left(\mathbf{k}^{\prime}+\mathbf{p}_{F}^{\omega}\right)\left[\mathbb{1}\left(\left\|\mathbf{k}^{\prime}\right\|<\left\|\mathbf{k}^{\prime}+\mathbf{p}_{F}^{\omega}-\mathbf{p}_{F}^{-\omega}\right\|\right)+\frac{1}{2} \mathbb{1}\left(\left\|\mathbf{k}^{\prime}\right\|=\left\|\mathbf{k}^{\prime}+\mathbf{p}_{F}^{\omega}-\mathbf{p}_{F}^{-\omega}\right\|\right)\right],
$$

where $\mathbf{p}_{F}^{\omega} \stackrel{\text { def }}{=}\left(0, \vec{p}_{F}^{\omega}\right)$. Note that, by the compact support properties of $\hat{g}_{\omega}^{(h)}\left(\mathbf{k}^{\prime}\right)$, if $\hat{g}_{\omega}^{(h)}\left(\mathbf{k}^{\prime}\right) \neq 0$, $h<0$, then $\hat{g}^{(j)}(\mathbf{k})=0$ for $|j-h|>1$, so that

$$
\hat{Q}_{\mathbf{k}^{\prime}, \omega, \rho, \rho^{\prime}}^{(h)}=1-\hat{W}_{2, \rho, \rho_{1},(\omega, \omega)}^{(h)}\left(\mathbf{k}^{\prime}\right) \hat{g}_{\omega, \rho_{1}, \rho_{2}}^{(h+1)}\left(\mathbf{k}^{\prime}\right) \hat{Q}_{\mathbf{k}^{\prime}, \omega, \rho_{2}, \rho^{\prime}}^{(h+1)},
$$

and, therefore, proceeding by induction, we see that on the support of $\hat{g}_{\omega}^{(h)}\left(\mathbf{k}^{\prime}\right)$ we have

$$
\left\|\hat{Q}_{\mathbf{k}^{\prime}, \omega}^{(h)}-1\right\| \leq C|U| \gamma^{\theta h}, \quad\left\|\partial_{\mathbf{k}^{\prime}}^{n} \hat{Q}_{\mathbf{k}^{\prime}, \omega}^{(h)}\right\| \leq C_{n}|U| \gamma^{(\theta-n) h} .
$$

In order to derive (3.112), we used Theorem 2 and the decay bounds (3.90).

Using (3.112), the definition (3.110) and the decay bounds (3.90), we find that

$$
\int d \mathbf{x}|\mathbf{x}|^{j}\left\|G_{\omega}^{(h)}(\mathbf{x})\right\| \leq C_{j} \gamma^{-(1+j) h} .
$$

Let us now prove (3.108). We proceed by induction. For $h=0$ (3.108) is clearly true (it coincides with (3.107)). Assuming inductively that the representation (3.108) is valid up to a certain value of $h<0$, we can show that the same representation is valid for $h-1$. In fact, we can rewrite the term $\mathcal{V}^{(h)}$ in the exponent of (3.108) as $\mathcal{V}^{(h)}=\mathcal{L V}^{(h)}+\mathcal{R} \mathcal{V}^{(h)}$, as in (3.60), and we can "absorb" $\mathcal{L} \mathcal{V}^{(h)}$ in the fermionic integration, as explained in Section IIIC see (3.63)-(3.65). Similarly we rewrite

$$
\frac{\partial}{\partial \Psi_{\mathbf{x}, \sigma, \rho, \omega}^{(\leq h) \pm}} \mathcal{V}^{(h)}\left(\Psi^{(\leq h)}\right)=\int d \mathbf{y} W_{2,\left(\rho, \rho^{\prime}\right),(\omega, \omega)}^{(h)}(\mathbf{x}, \mathbf{y}) \Psi_{\mathbf{y}, \sigma, \rho^{\prime}, \omega}^{(\leq h) \mp}+\frac{\partial}{\partial \Psi_{\mathbf{x}, \sigma, \rho, \omega}^{(\leq h) \pm}} \mathcal{R} \mathcal{V}^{(h)}\left(\Psi^{(\leq h)}\right)
$$

This rewriting induces a decomposition of the first line of (3.109) into two pieces, the first proportional to $W_{2}^{(h)}$, the second identical to the first line of (3.109) itself, with $\mathcal{V}^{(h)}$ replaced by $\mathcal{R} \mathcal{V}^{(h)}$, that we will call $\mathcal{R} B_{\phi}^{(h)}\left(\Psi^{(\leq h)}\right)$. We choose to "absorb" the term proportional to $W_{2}^{(h)}$ into the definition of $Q^{(h)}$, and this gives the recursion relation (3.111). Moreover, note that combining $\mathcal{R} B_{\phi}^{(h)}\left(\Psi^{(\leq h)}\right)$ with $\mathcal{R} \mathcal{V}^{(h)}\left(\Psi^{(\leq h)}\right)$ we find:

$$
\mathcal{R} \mathcal{V}^{(h)}\left(\Psi^{(\leq h)}\right)+\mathcal{R} B_{\phi}^{(h)}\left(\Psi^{(\leq h)}\right)=\mathcal{R} \mathcal{V}^{(h)}\left(\Psi^{(\leq h)}+G^{(h+1)} * \phi\right)+W_{R, 1}^{(h)},
$$


with $W_{R, 1}^{(h)}$ containing terms of third or higher order in $\phi$. We define $\bar{W}_{R}^{(h)}=W_{R}^{(h)}+W_{R, 1}^{(h)}$.

After these splittings and redefinitions, we can rewrite (3.108) as

$$
\begin{aligned}
& e^{\mathcal{W}(\phi)}=e^{-\beta|\Lambda|\left(F_{h}+e_{h}\right)+S^{(\geq h)}(\phi)} \int P_{\chi_{h-1}, A_{h-1}}\left(d \Psi^{(\leq h-1)}\right) \int P_{f_{h}^{-1}, \bar{A}_{h-1}}\left(d \Psi^{(h)}\right) \cdot \\
& \cdot e^{-\mathcal{R} \mathcal{V}^{(h)}\left(\Psi^{(\leq h)}+G^{(h+1)} * \phi\right)-\bar{W}_{R}^{(h)}+\int d \mathbf{k}^{\prime}\left[\hat{\phi}_{\mathbf{k}^{\prime}}^{+} \hat{Q}_{\mathbf{k}^{\prime}}^{(h)} \hat{\Psi}_{\mathbf{k}^{\prime}}^{(\leq h)-}+\hat{\Psi}_{\mathbf{k}^{\prime}}^{(\leq h)+} \hat{Q}_{\mathbf{k}^{\prime}}^{(h)} \hat{\phi}_{\mathbf{k}^{\prime}}^{-}\right]} .
\end{aligned}
$$

Integrating the field $\Psi^{(h)}$, we get the analogue of (3.73):

$$
\begin{gathered}
\int P_{f_{h}^{-1}, \bar{A}_{h-1}}\left(d \Psi^{(h)}\right) e^{-\mathcal{R} \mathcal{V}^{(h)}\left(\Psi^{(\leq h)}+G^{(h+1)} * \phi\right)-\bar{W}_{R}^{(h)}+\int d \mathbf{k}^{\prime}\left[\hat{\phi}_{\mathbf{k}^{\prime}}^{+} \hat{Q}_{\mathbf{k}^{\prime}}^{(h)} \hat{\Psi}_{\mathbf{k}^{\prime}}^{(\leq h)-}+\hat{\Psi}_{\mathbf{k}^{\prime}}^{(\leq h)+} \hat{Q}_{\mathbf{k}^{\prime}}^{(h)} \hat{\phi}_{\mathbf{k}^{\prime}}^{-}\right]}= \\
=e^{-\bar{e}_{h} \beta|\Lambda|-\mathcal{V}^{(h-1)}\left(\Psi^{(\leq h-1)}+G^{(h)} * \phi\right)+\int d \mathbf{k}^{\prime} \hat{\phi}_{\mathbf{k}^{\prime}}^{+} \hat{Q}_{\mathbf{k}^{\prime}}^{(h)} \hat{g}^{(h)}\left(\mathbf{k}^{\prime}\right) \hat{Q}_{\mathbf{k}^{\prime}}^{(h)} \hat{\phi}_{\mathbf{k}^{\prime}}^{-}-W_{R, 2}^{(h-1)} \cdot} \cdot(3.117) \\
\cdot e^{\int d \mathbf{k}^{\prime}\left[\hat{\phi}_{\mathbf{k}^{\prime}}^{+} \hat{Q}_{\mathbf{k}^{\prime}}^{(h)} \hat{\Psi}_{\mathbf{k}^{\prime}}^{(\leq h-1)-}+\hat{\Psi}_{\mathbf{k}^{\prime}}^{(\leq h-1)+} \hat{Q}_{\mathbf{k}^{\prime}}^{(h)} \hat{\phi}_{\mathbf{k}^{\prime}}^{-}\right]},
\end{gathered}
$$

with $G^{(h)}$ defined by the recursion relation (3.110) and $W_{R, 2}^{(h-1)}$ a term of third or higher order in $\phi$. Eq. (3.117) can be proved by making use of a formal change of Grassmann variables $\hat{\Psi}_{\mathbf{k}^{\prime}}^{\prime}=\hat{\Psi}_{\mathbf{k}^{\prime}}-\hat{g}^{(h)}\left(\mathbf{k}^{\prime}\right) Q_{\mathbf{k}^{\prime}}^{(h)} \hat{\phi}_{\mathbf{k}^{\prime}}$, as described in Ch.4 of [2]. At this point it is straightforward to check that the final expression for $e^{\mathcal{W}(\phi)}$ that we end up with is given by the r.h.s. of (3.108), with $h$ replaced by $h-1$, and the inductive assumption is proved.

From the definitions and the construction above, we get

$$
\begin{aligned}
& S_{\rho, \rho^{\prime}}(\mathbf{x}-\mathbf{y})=\sum_{\omega= \pm} e^{-i \vec{p}_{F}^{\omega}(\vec{x}-\vec{y})} S_{\omega, \rho, \rho^{\prime}}(\mathbf{x}-\mathbf{y}) \equiv \sum_{\omega= \pm} e^{-i \vec{p}_{F}^{\omega}(\vec{x}-\vec{y})} \\
& \cdot \sum_{h=-\infty}^{1}\left[\left(Q_{\omega, \rho, \rho_{1}}^{(h)} * g_{\omega, \rho_{1}, \rho_{2}}^{(h)} * Q_{\omega, \rho_{2}, \rho^{\prime}}^{(h)}\right)(\mathbf{x}-\mathbf{y})-\left(G_{\omega, \rho, \rho_{1}}^{(h)} * W_{2,\left(\rho_{1}, \rho_{2}\right),(\omega, \omega)}^{(h-1)} * G_{\omega, \rho_{2}, \rho^{\prime}}^{(h)}\right)(\mathbf{x}-\mathbf{y})\right]
\end{aligned}
$$

Analyticity of $S_{\rho, \rho^{\prime}}(\mathbf{x}-\mathbf{y})$ follows from this representation and the results of Theorem 2 . Concerning the representation (2.10), let us take the Fourier transform of $S_{\omega, \rho, \rho^{\prime}}(\mathbf{x}-\mathbf{y})$. If we define $h_{\mathbf{k}}=\min \left\{h: \hat{g}_{\omega}^{(h)}\left(\mathbf{k}^{\prime}\right) \not \equiv 0\right\}$, we get, for $\mathbf{k}^{\prime}$ inside the support of $\Psi_{\mathbf{k}^{\prime}, \sigma, \rho, \omega}^{(\leq 0), \rho, \rho^{\prime}}$

$$
\begin{aligned}
\hat{S}_{\omega, \rho, \rho^{\prime}}\left(\mathbf{k}^{\prime}\right) & =\sum_{j=h_{\mathbf{k}}}^{h_{\mathbf{k}}+1} Q_{\mathbf{k}^{\prime}, \omega, \rho, \rho_{1}}^{(j)} g_{\omega, \rho_{1}, \rho_{2}}^{(j)}\left(\mathbf{k}^{\prime}\right)^{(j)} Q_{\mathbf{k}^{\prime}, \omega, \rho_{2}, \rho^{\prime}}^{(j)}- \\
& -\sum_{j=h_{\mathbf{k}}}^{h_{\mathbf{k}}+1} G_{\omega, \rho, \rho_{1}}^{(j)}\left(\mathbf{k}^{\prime}\right) W_{2,\left(\rho_{1}, \rho_{2}\right),(\omega, \omega)}^{(j-1)}\left(\mathbf{k}^{\prime}\right) G_{\omega, \rho_{2}, \rho^{\prime}}^{(j)}\left(\mathbf{k}^{\prime}\right),
\end{aligned}
$$

which readily implies (2.10): in fact, using the explicit expression of $g_{\omega}^{(h)}$ and the inductive bounds on $Q^{(h)}$, see (3.112), it is easy to see that the term in the first line of (3.119) can be written as in (2.10) and that their only singularity is located at $\mathbf{k}^{\prime}=\mathbf{0}$.

The contributions from the second line can be bounded using the bounds on $W_{2}^{(h)}$ proved in Theorem 2, and we find that they can be bounded by $C|U| \gamma^{h_{\mathbf{k}^{\prime}}(-1+\theta)}$, which means that they only contribute to the error term appearing in (2.10). This also implies that no other singularity, besides the one at the Fermi points, can be produced by such terms.

Finally, if $\mathbf{k}$ does not belong to the support of $\Psi^{(\leq 0)}$, we can write

$$
\hat{S}_{\rho, \rho^{\prime}}(\mathbf{k})=\hat{S}_{\rho, \rho^{\prime}}^{(u . v .)}(\mathbf{k})=g^{(u . v .)}(\mathbf{x}-\mathbf{y})-\left(g_{\rho, \rho_{1}}^{(u . v .)} * W_{2,\left(\rho_{1}, \rho_{2}\right)} * g_{\rho_{2}, \rho^{\prime}}^{(u . v .)}\right)(\mathbf{x}-\mathbf{y}),
$$

with $W_{2, \underline{\rho}}$ defined by (3.35). The bounds discussed in Section 11 B and Appendix Вimply that $S_{\rho, \rho^{\prime}}^{(u . v)}(\mathbf{x}-\mathbf{y})$ decays faster than any power, so that no singularity can appear in its Fourier transform.

A similar expansion can be obtained for higher order Schwinger functions, but we will not belabor the details here. This concludes the proof of Theorem 1 . 


\section{APPENDIX A: THE NON-INTERACTING THEORY}

In this Appendix we give some details about the computation of the Schwinger functions of the non interacting theory, i.e., of model (2.1) with $U=0$. In this case the Hamiltonian of interest reduces to

$$
H_{0, \Lambda}=-\sum_{\substack{\vec{x} \in \Lambda \\ i=1,2,3}} \sum_{\sigma=\uparrow \downarrow}\left(a_{\vec{x}, \sigma}^{+} b_{\vec{x}+\vec{\delta}_{i}, \sigma}^{-}+b_{\vec{x}+\vec{\delta}_{i}, \sigma}^{+} a_{\vec{x}, \sigma}^{-}\right)
$$

with $\Lambda, a_{\vec{x}, \sigma}^{ \pm}, b_{\vec{x}+\vec{\delta}_{i}, \sigma}^{ \pm}$defined as in items (1)-(4) after (2.1).

First of all, let us remind that, being $H_{0, \Lambda}$ quadratic, the $2 n$-point Schwinger functions satisfy the Wick rule, i.e.,

$$
\begin{aligned}
& \left\langle\mathbf{T}\left\{\Psi_{\mathbf{x}_{1}, \sigma_{1}, \rho_{1}}^{-} \cdots \Psi_{\mathbf{x}_{n}, \sigma_{n}, \rho_{n}}^{-} \Psi_{\mathbf{y}_{1}, \sigma_{1}^{\prime}, \rho_{1}^{\prime}}^{+} \cdots \Psi_{\mathbf{y}_{n}, \sigma_{n}^{\prime}, \rho_{n}^{\prime}}^{+}\right\}\right\rangle_{\beta, \Lambda}=-\operatorname{det} G, \\
& G_{i j}=\delta_{\sigma_{i} \sigma_{j}^{\prime}}\left\langle\mathbf{T}\left\{\Psi_{\mathbf{x}_{i}, \sigma_{i}, \rho_{i}}^{-} \Psi_{\mathbf{y}_{j}, \sigma_{j}^{\prime}, \rho_{j}^{\prime}}^{+}\right\}\right\rangle_{\beta, \Lambda} .
\end{aligned}
$$

Moreover, every $n$-point Schwinger function $S_{n}^{\beta, \Lambda}\left(\mathbf{x}_{1}, \varepsilon_{1}, \sigma_{1}, \rho_{1} ; \ldots ; \mathbf{x}_{n}, \varepsilon_{n}, \sigma_{n}, \rho_{n}\right)$ with $\sum_{i=1}^{n} \varepsilon_{i} \neq 0$ is identically zero. Therefore, in order to construct the whole set of Schwinger functions of $H_{0, \Lambda}$, it is enough to compute the 2-point function $S_{0}^{\beta, \Lambda}(\mathbf{x}-\mathbf{y})=\left\langle\mathbf{T}\left\{\Psi_{\mathbf{x}, \sigma, \rho}^{-} \Psi_{\mathbf{y}, \sigma, \rho^{\prime}}^{+}\right\}\right\rangle_{\beta, \Lambda}$, and in order to do this, it is convenient to first diagonalize $H_{0, \Lambda}$. Let us proceed as follows.

We identify $\Lambda$ with the set of vectors in a fundamental cell, and we write

$$
\Lambda=\left\{n_{1} \vec{a}_{1}+n_{2} \vec{a}_{2}: 0 \leq n_{1}, n_{2} \leq L-1\right\},
$$

with $\vec{a}_{1}=\frac{1}{2}(3, \sqrt{3})$ and $\vec{a}_{2}=\frac{1}{2}(3,-\sqrt{3})$. The reciprocal lattice $\Lambda^{*}$ is the set of vectors such that $e^{i \vec{K} \vec{x}}=1$, if $\vec{x} \in \Lambda$. A basis $\vec{b}_{1}, \vec{b}_{2}$ for $\Lambda^{*}$ can be obtained by the inversion formula:

$$
\left(\begin{array}{ll}
b_{11} & b_{12} \\
b_{21} & b_{22}
\end{array}\right)=2 \pi\left(\begin{array}{ll}
a_{11} & a_{21} \\
a_{12} & a_{22}
\end{array}\right)^{-1}
$$

which gives

$$
\vec{b}_{1}=\frac{2 \pi}{3}(1, \sqrt{3}), \quad \vec{b}_{2}=\frac{2 \pi}{3}(1,-\sqrt{3})
$$

We call $\mathcal{D}_{L}$ the set of quasi-momenta $\vec{k}$ of the form

$$
\vec{k}=\frac{m_{1}}{L} \vec{b}_{1}+\frac{m_{2}}{L} \vec{b}_{2}, \quad m_{1}, m_{2} \in \mathbb{Z},
$$

identified modulo $\Lambda^{*}$; this means that $\mathcal{D}_{L}$ can be identified with the vectors $\vec{k}$ of the form (2.2) and restricted to the first Brillouin zone:

$$
\mathcal{D}_{L}=\left\{\vec{k}=\frac{m_{1}}{L} \vec{b}_{1}+\frac{m_{2}}{L} \vec{b}_{2}: 0 \leq m_{1}, m_{2} \leq L-1\right\} .
$$

Given a periodic function $f: \Lambda \rightarrow \mathbb{R}$, its Fourier transform is defined as

$$
f(\vec{x})=\frac{1}{|\Lambda|} \sum_{\vec{k} \in \mathcal{D}_{L}} e^{i \vec{k} \vec{x}} \hat{f}(\vec{k})
$$

which can be inverted into

$$
\hat{f}(\vec{k})=\sum_{\vec{x} \in \Lambda} e^{-i \vec{k} \vec{x}} f(\vec{x}), \quad \vec{k} \in \mathcal{D}_{L}
$$

where we used the identity

$$
\sum_{\vec{x} \in \Lambda} e^{i \vec{k} \vec{x}}=|\Lambda| \delta_{\vec{k}, \overrightarrow{0}}
$$


and $\delta$ is the periodic Kronecker delta function over $\Lambda^{*}$.

We now associate to the set of creation/annihilation operators $a_{\vec{x}, \sigma}^{ \pm}, b_{\vec{x}+\vec{\delta}_{i}, \sigma}^{ \pm}$the corresponding set of operators in momentum space:

$$
a_{\vec{x}, \sigma}^{ \pm}=\frac{1}{|\Lambda|} \sum_{\vec{k} \in \mathcal{D}_{L}} e^{ \pm i \vec{k} \vec{x}} \hat{a}_{\vec{k}, \sigma}^{ \pm}, \quad \quad b_{\vec{x}+\vec{\delta}_{1}, \sigma}^{ \pm}=\frac{1}{|\Lambda|} \sum_{\vec{k} \in \mathcal{D}_{L}} e^{ \pm i \vec{k} \vec{x}} \hat{b}_{\vec{k}, \sigma}^{ \pm} .
$$

Note that, using (A[8)-(A|10), we find that

$$
\hat{a}_{\vec{k}, \sigma}^{ \pm}=\sum_{\vec{x} \in \Lambda} e^{\mp i \vec{k} \vec{x}} a_{\vec{x}, \sigma}^{ \pm}, \quad \quad \hat{b}_{\vec{k}, \sigma}^{ \pm}=\sum_{\vec{x} \in \Lambda} e^{\mp i \vec{k} \vec{x}} b_{\vec{x}+\vec{\delta}_{1}, \sigma}^{ \pm}
$$

are fermionic creation/annihilation operators satisfying

$$
\left\{a_{\vec{k}, \sigma}^{\varepsilon}, a_{\vec{k}^{\prime}, \sigma^{\prime}}^{\varepsilon^{\prime}}\right\}=|\Lambda| \delta_{\vec{k}, \vec{k}^{\prime}} \delta_{\varepsilon,-\varepsilon^{\prime}} \delta_{\sigma, \sigma^{\prime}}, \quad\left\{b_{\vec{k}, \sigma}^{\varepsilon}, \quad b_{\vec{k}^{\prime}, \sigma^{\prime}}^{\varepsilon^{\prime}}\right\}=|\Lambda| \delta_{\vec{k}, \overrightarrow{k^{\prime}}} \delta_{\varepsilon,-\varepsilon^{\prime}} \delta_{\sigma, \sigma^{\prime}}
$$

and $\left\{a_{\vec{k}, \sigma}^{\varepsilon}, b_{\vec{k}^{\prime}, \sigma^{\prime}}^{\varepsilon^{\prime}}\right\}=0$. With these definitions, we can rewrite

$$
\begin{aligned}
& H_{0, \Lambda}=-\sum_{\substack{\vec{x} \in \Lambda \\
i=1,2,3}} \sum_{\sigma=\uparrow \downarrow}\left(a_{\vec{x}, \sigma}^{+} b_{\vec{x}+\vec{\delta}_{i}, \sigma}^{-}+b_{\vec{x}+\vec{\delta}_{i}, \sigma}^{+} a_{\vec{x}, \sigma}^{-}\right)= \\
= & -\frac{1}{|\Lambda|^{2}} \sum_{\substack{\vec{x} \in \Lambda \\
i=1,2,3}} \sum_{\sigma=\uparrow \downarrow \vec{k}, \vec{k}^{\prime} \in \mathcal{D}_{L}}\left(e^{+i \vec{k} \vec{x}} e^{-i \vec{k}^{\prime}\left(\vec{x}+\vec{\delta}_{i}-\vec{\delta}_{1}\right)} \hat{a}_{\vec{k}, \sigma}^{+} \hat{b}_{\vec{k}^{\prime}, \sigma}^{-}+e^{-i \vec{k} \vec{x}} e^{+i \vec{k}^{\prime}\left(\vec{x}+\vec{\delta}_{i}-\vec{\delta}_{1}\right)} \hat{b}_{\vec{k}^{\prime}, \sigma}^{+} \hat{a}_{\vec{k}, \sigma}^{-}\right)= \\
= & -\frac{1}{|\Lambda|} \sum_{\vec{k} \in \mathcal{D}_{L}} \sum_{\sigma=\uparrow \downarrow}\left(v_{\vec{k}}^{*} \hat{a}_{\vec{k}, \sigma}^{+} \hat{b}_{\vec{k}, \sigma}^{-}+v_{\vec{k}} \hat{b}_{\vec{k}, \sigma}^{+} \hat{a}_{\vec{k}, \sigma}^{-}\right),
\end{aligned}
$$

with

$$
v_{\vec{k}}=\sum_{i=1}^{3} e^{i\left(\vec{\delta}_{i}-\vec{\delta}_{1}\right) \vec{k}}=1+2 e^{-i \frac{3}{2} k_{1}} \cos \frac{\sqrt{3}}{2} k_{2} .
$$

The Hamiltonian $H_{0, \Lambda}$ can be diagonalized by introducing the fermionic operators

$$
\hat{\alpha}_{\vec{k}, \sigma}=\frac{\hat{a}_{\vec{k}, \sigma}}{\sqrt{2}}+\frac{v_{\vec{k}}^{*}}{\sqrt{2}\left|v_{\vec{k}}\right|} \hat{b}_{\vec{k}, \sigma}, \quad \hat{\beta}_{\vec{k}, \sigma}=\frac{\hat{a}_{\vec{k}, \sigma}}{\sqrt{2}}-\frac{v_{\vec{k}}^{*}}{\sqrt{2}\left|v_{\vec{k}}\right|} \hat{b}_{\vec{k}, \sigma},
$$

in terms of which we can re-write

$$
H_{0, \Lambda}=\frac{1}{|\Lambda|} \sum_{\vec{k} \in \mathcal{D}_{L}} \sum_{\sigma=\uparrow \downarrow}\left(-\left|v_{\vec{k}}\right| \hat{\alpha}_{\vec{k}, \sigma}^{+} \hat{\alpha}_{\vec{k}, \sigma}+\left|v_{\vec{k}}\right| \hat{\beta}_{\vec{k}, \sigma}^{+} \hat{\beta}_{\vec{k}, \sigma}\right)
$$

with

$$
\left|v_{\vec{k}}\right|=\sqrt{\left(1+2 \cos \left(3 k_{1} / 2\right) \cos \left(\sqrt{3} k_{2} / 2\right)\right)^{2}+4 \sin ^{2}\left(3 k_{1} / 2\right) \cos ^{2}\left(\sqrt{3} k_{2} / 2\right)},
$$

which is vanishing iff $\vec{k}=\vec{p}_{F}^{\omega}, \omega= \pm$, with

$$
\vec{p}_{F}^{\omega}=\left(\frac{2 \pi}{3}, \omega \frac{2 \pi}{3 \sqrt{3}}\right) .
$$

Now, for $\vec{x} \in \Lambda$, we define $\alpha_{\vec{x}, \sigma}^{ \pm}=|\Lambda|^{-1} \sum_{\vec{k} \in \mathcal{D}_{L}} e^{ \pm i \vec{k} \vec{x}} \hat{\alpha}_{\vec{k}, \sigma}$ and $\beta_{\vec{x}, \sigma}^{ \pm}=|\Lambda|^{-1} \sum_{\vec{k} \in \mathcal{D}_{L}} e^{ \pm i \vec{k} \vec{x}} \hat{\alpha}_{\vec{k}, \sigma}$; moreover, if $\mathbf{x}=\left(x_{0}, \vec{x}\right)$ we define $\alpha_{\mathbf{x}, \sigma}^{ \pm}=e^{H_{0, \Lambda} x_{0}} \alpha_{\vec{x}, \sigma}^{ \pm} e^{-H_{0, \Lambda} x_{0}}$ and $\beta_{\mathbf{x}, \sigma}^{ \pm}=e^{H_{0, \Lambda} x_{0}} \beta_{\vec{x}, \sigma}^{ \pm} e^{-H_{0, \Lambda} x_{0}}$. A straightforward computation, see, e.g., Appendix 1 of [2], shows that, if $-\beta<x_{0}-y_{0} \leq \beta$,

$$
\begin{aligned}
& \left\langle\mathbf{T}\left\{\alpha_{\mathbf{x}, \sigma}^{-} \alpha_{\mathbf{y}, \sigma^{\prime}}^{+}\right\}\right\rangle_{\beta, \Lambda}= \\
& =\frac{\delta_{\sigma, \sigma^{\prime}}}{|\Lambda|} \sum_{\vec{k} \in \mathcal{D}_{L}} e^{-i \vec{k}(\vec{x}-\vec{y})}\left[\mathbb{1}\left(x_{0}-y_{0}>0\right) \frac{e^{\left(x_{0}-y_{0}\right)\left|v_{\vec{k}}\right|}}{1+e^{\beta\left|v_{\vec{k}}\right|}}-\mathbb{1}\left(x_{0}-y_{0} \leq 0\right) \frac{e^{\left(x_{0}-y_{0}+\beta\right)\left|v_{\vec{k}}\right|}}{1+e^{\beta\left|v_{\vec{k}}\right|}}\right]
\end{aligned}
$$




$$
\begin{aligned}
& \left\langle\mathbf{T}\left\{\beta_{\mathbf{x}, \sigma}^{-} \beta_{\mathbf{y}, \sigma^{\prime}}^{+}\right\}\right\rangle_{\beta, \Lambda}= \\
& =\frac{\delta_{\sigma, \sigma^{\prime}}}{|\Lambda|} \sum_{\vec{k} \in \mathcal{D}_{L}} e^{-i \vec{k}(\vec{x}-\vec{y})}\left[\mathbb{1}\left(x_{0}-y_{0}>0\right) \frac{e^{-\left(x_{0}-y_{0}\right)\left|v_{\vec{k}}\right|}}{1+e^{-\beta\left|v_{\vec{k}}\right|}}-\mathbb{1}\left(x_{0}-y_{0} \leq 0\right) \frac{e^{-\left(x_{0}-y_{0}+\beta\right)\left|v_{\vec{k}}\right|}}{1+e^{-\beta\left|v_{\vec{k}}\right|}}\right]
\end{aligned}
$$

and $\left\langle\mathbf{T}\left\{\alpha_{\mathbf{x}, \sigma}^{-} \beta_{\mathbf{y}, \sigma^{\prime}}^{+}\right\}\right\rangle_{\beta, \Lambda}=\left\langle\mathbf{T}\left\{\beta_{\mathbf{x}, \sigma}^{-} \alpha_{\mathbf{y}, \sigma^{\prime}}^{+}\right\}\right\rangle_{\beta, \Lambda}=0$. A priori Eq. Al21 and (Al22) are defined only for $-\beta<x_{0}-y_{0} \leq \beta$, but we can extend them periodically over the whole real axis; the periodic extension of the propagator is continuous in the time variable for $x_{0}-y_{0} \notin \beta \mathbb{Z}$, and it has jump discontinuities at the points $x_{0}-y_{0} \in \beta \mathbb{Z}$. Note that at $x_{0}-y_{0}=\beta n$, the difference between the right and left limits is equal to $(-1)^{n} \delta_{\vec{x}, \vec{y}}$, so that the propagator is discontinuous only at $\mathbf{x}-\mathbf{y}=\beta \mathbb{Z} \times \overrightarrow{0}$. For $\mathbf{x}-\mathbf{y} \notin \beta \mathbb{Z} \times \overrightarrow{0}$, we can write

$$
\begin{aligned}
\left\langle\mathbf{T}\left\{\alpha_{\mathbf{x}, \sigma}^{-} \alpha_{\mathbf{y}, \sigma^{\prime}}^{+}\right\}\right\rangle_{\beta, \Lambda} & =\lim _{M \rightarrow \infty} \frac{\delta_{\sigma, \sigma^{\prime}}}{\beta|\Lambda|} \sum_{\mathbf{k} \in \mathcal{D}_{\beta, L}} e^{-i \mathbf{k}(\mathbf{x}-\mathbf{y})} \frac{1}{-i k_{0}-\left|v_{\vec{k}}\right|}, \\
\left\langle\mathbf{T}\left\{\beta_{\mathbf{x}, \sigma}^{-} \beta_{\mathbf{y}, \sigma^{\prime}}^{+}\right\}\right\rangle_{\beta, \Lambda} & =\lim _{M \rightarrow \infty} \frac{\delta_{\sigma, \sigma^{\prime}}}{\beta|\Lambda|} \sum_{\mathbf{k} \in \mathcal{D}_{\beta, L}} e^{-i \mathbf{k}(\mathbf{x}-\mathbf{y})} \frac{1}{-i k_{0}+\left|v_{\vec{k}}\right|} .
\end{aligned}
$$

Note indeed that for $x_{0}-y_{0} \notin \beta \mathbb{Z}$ the sums over $k_{0}$ in $\mathrm{A}[22$ are convergent, uniformly in $M$; if $x_{0}-y_{0}=\beta n$ and $\vec{x} \neq \vec{y}$, the r.h.s. of $(\underline{A \mid 22}$ is equal to

$$
\begin{array}{r}
\frac{1}{2}\left(\lim _{x_{0}-y_{0} \rightarrow(\beta n)^{+}}\left\langle\mathbf{T}\left\{\alpha_{\mathbf{x}, \sigma^{-}}^{-} \alpha_{\mathbf{y}, \sigma^{\prime}}^{+}\right\}\right\rangle_{\beta, \Lambda}+\lim _{x_{0}-y_{0} \rightarrow(\beta n)^{-}}\left\langle\mathbf{T}\left\{\alpha_{\mathbf{x}, \sigma^{-}}^{-} \alpha_{\mathbf{y}, \sigma^{\prime}}^{+}\right\}\right\rangle_{\beta, \Lambda}\right)= \\
=\left.\left\langle\mathbf{T}\left\{\alpha_{\mathbf{x}, \sigma^{-}}^{-} \alpha_{\mathbf{y}, \sigma^{\prime}}^{+}\right\}\right\rangle_{\beta, \Lambda}\right|_{x_{0}-y_{0}=\beta n} .
\end{array}
$$

A similar remark is valid for $\left\langle\mathbf{T}\left\{\beta_{\mathbf{x}, \sigma}^{-} \beta_{\mathbf{y}, \sigma^{\prime}}^{+}\right\}\right\rangle_{\beta, \Lambda}$. If we now re-express $\alpha_{\mathbf{x}, \sigma}^{ \pm}$and $\beta_{\mathbf{x}, \sigma}^{ \pm}$in terms of $a_{\mathbf{x}, \sigma}^{ \pm}$and $b_{\mathbf{x}+\boldsymbol{\delta}_{1}, \sigma}^{ \pm}$, using (A[16), we get (2.6). Note finally that if $\mathbf{x}=\mathbf{y}$

$$
\lim _{M \rightarrow \infty} \frac{1}{\beta|\Lambda|} \sum_{\mathbf{k} \in \mathcal{D}_{\beta, L}} \hat{g}_{\mathbf{k}}=\sum_{\mathbf{k} \in \mathcal{D}_{\beta, L}} \frac{1}{k_{0}^{2}+|v(\vec{k})|^{2}}\left(\begin{array}{cc}
0 & -v^{*}(\vec{k}) \\
-v(\vec{k}) & 0
\end{array}\right),
$$

so that the diagonal part is vanishing; on the contrary, using (A] 16 and the fact that $\left\langle\hat{\alpha}_{\vec{k}, \sigma^{\prime}}^{+} \hat{\beta}_{\vec{k}^{\prime}, \sigma^{\prime}}\right\rangle_{\beta, \Lambda}=\left\langle\hat{\beta}_{\vec{k}, \sigma}^{+} \hat{\alpha}_{\vec{k}^{\prime}, \sigma^{\prime}}\right\rangle_{\beta, \Lambda}=0$, we get

$$
\begin{aligned}
S_{0}\left(0^{-}, \overrightarrow{0}\right)_{1,1} & =S_{0}\left(0^{-}, \overrightarrow{0}\right)_{2,2}=-\frac{1}{2}\left(\left\langle\alpha_{\vec{x}, \sigma}^{+} \alpha_{\vec{x}, \sigma}\right\rangle_{\beta, \Lambda}+\left\langle\beta_{\vec{x}, \sigma}^{+} \beta_{\vec{x}, \sigma}\right\rangle_{\beta, \Lambda}\right)= \\
& =-\frac{1}{2|\Lambda|} \sum_{\vec{k} \in \mathcal{D}_{L}}\left(\frac{e^{\beta\left|v_{\vec{k}}\right|}}{1+e^{\beta\left|v_{\vec{k}}\right|}}+\frac{e^{-\beta\left|v_{\vec{k}}\right|}}{1+e^{-\beta\left|v_{\vec{k}}\right|}}\right)=-\frac{1}{2},
\end{aligned}
$$

and this explains why there are no quadratic terms in $V(\Psi)$, see (3.19).

\section{APPENDIX B: THE ULTRAVIOLET INTEGRATION}

In order to prove Eq.(3.35) -3.37), a simple application of (3.80) and determinant bounds is not enough, because $g^{(u . v .)}(\mathbf{x})$ does not admit a Gram representation, which is a key property needed for the implementation of standard fermionic cluster expansion methods. As mentioned in Section IIIB, a way out of this problem is to decompose the ultraviolet propagator into a sum of propagators, each admitting a Gram representation, and performing a simple multiscale analysis of the ultraviolet problem, in analogy with the standard strategy for ultraviolet problems in fermionic Quantum Field Theories [9, 15]. This multiscale analysis is very similar to (but much simpler than) the one describe in Section IIIC it has been performed in several previous papers [3, 4, 5] and it is reported here for completeness.

Let $M$ be the integer introduced after (2.6), and let us write

$$
g^{(u . v .)}(\mathbf{x})=\sum_{h=1}^{h_{M}} g^{(h)}(\mathbf{x})
$$


where

$$
g^{(h)}(\mathbf{x})=\frac{1}{\beta|\Lambda|} \sum_{\mathbf{k} \in \mathcal{D}_{\beta, L}} f_{u . v .}(\mathbf{k}) H_{h}\left(k_{0}\right) e^{-i \mathbf{k x}} \hat{g}_{\mathbf{k}}
$$

with $H_{1}\left(k_{0}\right)=\chi_{0}\left(\left|k_{0}\right|\right), H_{h}\left(k_{0}\right)=\chi_{0}\left(\gamma^{-h+1}\left|k_{0}\right|\right)-\chi_{0}\left(\gamma^{-h+2}\left|k_{0}\right|\right)$ and $h_{M}$ is the smallest integer such that $f_{u . v .}(\mathbf{k}) H_{j}\left(k_{0}\right) \equiv 0$ for all $j>h_{M}$ (note that $\left.h_{M} \simeq \log (M / \beta)\right)$. Note that $g^{(h)}(\mathbf{0})=0$ and, for any integer $K \geq 0, g^{(h)}(\mathbf{x})$ satisfies the bound

$$
\left|g^{(h)}(\mathbf{x})\right| \leq \frac{C_{K}}{1+\left(\gamma^{h}\left|x_{0}\right|_{\beta}+|\vec{x}|_{\Lambda}\right)^{K}}
$$

where $|\cdot|_{\beta}$ is the distance from the origin on the one dimensional torus of size $\beta$, while $|\cdot|_{\Lambda}$ is the distance on $\Lambda$. Moreover, $g^{(h)}(\mathbf{x})$ admits a Gram representation: $g^{(h)}(\mathbf{x}-\mathbf{y})=\int d \mathbf{z} A_{h}^{*}(\mathbf{x}-$ $\mathbf{z}) \cdot B_{h}(\mathbf{y}-\mathbf{z})$, with

$$
\begin{aligned}
& A_{h}(\mathbf{x})=\frac{1}{\beta|\Lambda|} \sum_{\mathbf{k} \in \mathcal{D}_{\beta, L}} \sqrt{f_{u . v .}(\mathbf{k}) H_{h}\left(k_{0}\right)} \frac{e^{-i \mathbf{k x}}}{k_{0}^{2}+|v(\vec{k})|^{2}}\left(\begin{array}{cc}
1 & 0 \\
0 & 1
\end{array}\right), \\
& B_{h}(\mathbf{x})=\frac{1}{\beta|\Lambda|} \sum_{\mathbf{k} \in \mathcal{D}_{\beta, L}} \sqrt{f_{u . v .}(\mathbf{k}) H_{h}\left(k_{0}\right)} e^{-i \mathbf{k x}}\left(\begin{array}{cc}
i k_{0} & -v^{*}(\vec{k}) \\
-v(\vec{k}) & i k_{0}
\end{array}\right)
\end{aligned}
$$

and

$$
\left\|A_{h}\right\|^{2}=\int d \mathbf{z}\left|A_{h}(\mathbf{z})\right|^{2} \leq C \gamma^{-3 h}, \quad\left\|B_{h}\right\|^{2} \leq C \gamma^{3 h},
$$

for a suitable constant $C$.

Our goal is to compute

$$
e^{-\beta|\Lambda| F_{0}-\mathcal{V}\left(\Psi^{(i . r)}\right)}=\lim _{M \rightarrow \infty} \int P\left(d \Psi^{\left[1, h_{M}\right]}\right) e^{V\left(\Psi^{(i . r .)}+\Psi^{\left[1, h_{M}\right]}\right)},
$$

where $P\left(d \Psi^{\left[1, h_{M}\right]}\right)$ is the fermionic "Gaussian integration" associated with the propagator $\sum_{h=1}^{h_{M}} \hat{g}^{(h)}(\mathbf{k})$ (i.e., it is the same as $P\left(d \Psi^{(u . v .)}\right)$ ). We perform the integration of (B],6) in an iterative fashion, analogous to the procedure described in Section IIIC for the infrared integration. We can inductively prove the analogue of (3.57), i.e.,

$$
e^{-\beta|\Lambda| F_{0}-\mathcal{V}\left(\Psi^{(i . r)}\right)}=e^{-\beta|\Lambda| F_{h}} \int P\left(d \Psi^{[1, h]}\right) e^{\mathcal{V}^{(h)}\left(\Psi^{(i . r .)}+\Psi^{[1, h]}\right)}
$$

where $P\left(d \Psi^{[1, h]}\right)$ is the fermionic "Gaussian integration" associated with the propagator $\sum_{h=1}^{h} \hat{g}^{(h)}(\mathbf{k})$ and

$$
\mathcal{V}^{(h)}\left(\Psi^{[1, h]}\right)=\sum_{n=1}^{\infty} \sum_{\underline{\rho}, \underline{\underline{\sigma}}} \int d \mathbf{x}_{1} \cdots d \mathbf{x}_{2 n}\left[\prod_{j=1}^{n} \Psi_{\mathbf{x}_{2 j-1}, \sigma_{j}, \rho_{2 j-1}}^{[1, h]+} \Psi_{\mathbf{x}_{2 j}, \sigma_{j}, \rho_{2 j}}^{[1, h]--}\right] W_{2 n, \underline{\rho}}^{(h)}\left(\mathbf{x}_{1}, \ldots, \mathbf{x}_{2 n}\right) .
$$

In order to inductively prove $(\mathrm{B}, 7)-(\mathrm{B}, 8)$ we simply use the addition principle to rewrite

$$
\int P\left(d \Psi^{[1, h]}\right) e^{\mathcal{V}^{(h)}\left(\Psi^{(i . r .)}+\Psi^{[1, h]}\right)}=\int P\left(d \Psi^{[1, h-1]}\right) \int P\left(d \Psi^{(h)}\right) e^{\mathcal{V}^{(h)}\left(\Psi^{(i . r .)}+\Psi^{[1, h-1]}+\Psi^{(h)}\right)},
$$

where $P\left(d \Psi^{(h)}\right)$ is the fermionic Gaussian integration with propagator $\hat{g}^{(h)}(\mathbf{k})$. After the integration of $\left.\Psi^{(h)}\right)$ we define

$$
e^{\mathcal{V}^{(h-1)}\left(\Psi^{(i . r .)}+\Psi^{[1, h-1]}\right)-\beta|\Lambda| \bar{e}_{h}}=\int P\left(d \Psi^{(h)}\right) e^{\mathcal{V}^{(h)}\left(\Psi^{(i . r .)}+\Psi^{[1, h-1]}+\Psi^{(h)}\right)},
$$


which proves (B/7). In analogy with (3.74) we have

$$
\bar{e}_{h}+\mathcal{V}^{(h-1)}(\Psi)=\sum_{n \geq 1} \frac{1}{n !}(-1)^{n+1} \mathcal{E}_{h}^{T}\left(\mathcal{V}^{(h)}\left(\Psi+\Psi^{(h)}\right) ; n\right)
$$

As described in Section \IC, the iterative action of $\mathcal{E}_{h_{i}}^{T}$ can be conveniently represented in terms of trees $\tau \in \mathcal{T}_{M ; h, n}$, where $\mathcal{T}_{M ; h, n}$ is a set of labelled trees, completely analogous to the set $\mathcal{T}_{h, n}$ described before Eq.(3.75), unless for the following modifications:

1. a tree $\tau \in \mathcal{T}_{M ; h, n}$ has vertices $v$ associated with scale labels $h+1 \leq h_{v} \leq h_{M}+1$, while the root $r$ has scale $h$;

2. with each end-point $v$ we associate $V\left(\Psi^{\left[1, h_{M}\right]}\right)$, with $V(\Psi)$ defined in (3.19).

In terms of these trees, the effective potential $\mathcal{V}^{(h)}, 0 \leq h \leq h_{M}$ (with $\mathcal{V}^{(0)}\left(\Psi^{(i . r .)}\right)$ identified with $\left.\mathcal{V}\left(\Psi^{(i . r .)}\right)\right)$, can be written as

$$
\mathcal{V}^{(h)}\left(\Psi^{[1, h]}\right)+\beta|\Lambda| \bar{e}_{h+1}=\sum_{n=1}^{\infty} \sum_{\tau \in \mathcal{T}_{M ; h, n}} \mathcal{V}^{(h)}\left(\tau, \Psi^{[1, h]}\right)
$$

where, if $v_{0}$ is the first vertex of $\tau$ and $\tau_{1}, \ldots, \tau_{s}\left(s=s_{v_{0}}\right)$ are the subtrees of $\tau$ with root $v_{0}$, $\mathcal{V}^{(h)}\left(\tau, \Psi^{[1, h]}\right)$ is defined inductively as follows:

i) if $s>1$, then

$$
\mathcal{V}^{(h)}\left(\tau, \Psi^{[1, h]}\right)=\frac{(-1)^{s+1}}{s !} \mathcal{E}_{h+1}^{T}\left[\overline{\mathcal{V}}^{(h+1)}\left(\tau_{1}, \Psi^{[1, h+1]}\right) ; \ldots ; \overline{\mathcal{V}}^{(h+1)}\left(\tau_{s}, \Psi^{[1, h+1]}\right)\right]
$$

where $\overline{\mathcal{V}}^{(h+1)}\left(\tau_{i}, \Psi^{[1, h+1]}\right)$ is equal to $\mathcal{V}^{(h+1)}\left(\tau_{i}, \Psi^{[1, h+1]}\right)$ if the subtree $\tau_{i}$ contains more than one end-point, or if it contains one end-point but it is not a trivial subtree; it is equal to $V\left(\Psi^{[1, h+1]}\right)$ if $\tau_{i}$ is a trivial subtree;

ii) if $s=1$, then $\mathcal{V}^{(h)}\left(\tau, \Psi^{(\leq h)}\right)$ is equal to $\mathcal{E}_{h+1}^{T}\left[\mathcal{V}^{(h+1)}\left(\tau_{1}, \Psi^{[1, h+1]}\right)\right]$ if $\tau_{1}$ is not a trivial subtree; it is equal to $\mathcal{E}_{h+1}^{T}\left[V\left(\Psi^{[1, h+1]}\right)-V\left(\Psi^{[1, h]}\right)\right]$ if $\tau_{1}$ is a trivial subtree.

Note that, with $V(\Psi)$ defined as in 3.19 and with the present choice of the ultraviolet cutoff ( such that $\left.g^{(h)}(\mathbf{0})=0\right)$, we get $\mathcal{E}_{h+1}^{T}\left[V\left(\Psi^{[1, h+1]}\right)-V\left(\Psi^{[1, h]}\right)\right]=0$. This implies that, if $v$ is not an endpoint and $n(v)$ is the number of endpoints following $v$ on $\tau$, and if $\tau$ has a vertex $v$ with $n(v)=1$, then its value vanishes: therefore, in the sum over the trees, we can freely impose the constraint that $n(v)>1$ for all vertices $v \in \tau$. From now on we shall assume that the trees in $\mathcal{T}_{M ; h, n}$ satisfy this constraint.

Repeating step by step the discussion leading to (3.77), (3.85) and (3.86), and using analogous definitions, we find that

$$
\mathcal{V}^{(h)}(\tau, \mathbf{P})=\sum_{T \in \mathbf{T}} \int d \mathbf{x}_{v_{0}} \widetilde{\Psi}^{(\leq h)}\left(P_{v_{0}}\right) W_{\tau, \mathbf{P}, T}^{(h)}\left(\mathbf{x}_{v_{0}}\right) \equiv \sum_{T \in \mathbf{T}} \mathcal{V}^{(h)}(\tau, \mathbf{P}, T),
$$

where

$$
\widetilde{\Psi}^{(\leq h)}\left(P_{v}\right)=\prod_{f \in P_{v}} \Psi_{\mathbf{x}(f), \sigma(f), \rho(f)}^{(\leq h) \varepsilon(f)}
$$

and

$W_{\tau, \mathbf{P}, T}\left(\mathbf{x}_{v_{0}}\right)=U^{n}\left\{\prod_{\substack{v \\ \text { not e.p. }}} \frac{1}{s_{v} !} \int d P_{T_{v}}\left(\mathbf{t}_{v}\right) \operatorname{det} G^{h_{v}, T_{v}}\left(\mathbf{t}_{v}\right)\left[\prod_{l \in T_{v}} \delta_{\sigma_{l}^{-}, \sigma_{l}^{+}}\left[g^{(h)}\left(\mathbf{x}_{l}-\mathbf{y}_{l}\right)\right]_{\rho_{l}^{-}, \rho_{l}^{+}}\right]\right\}$.

Moreover, $G^{h_{v}, T_{v}}\left(\mathbf{t}_{v}\right)$ is a matrix, analogous to (3.81), with $\delta_{\omega_{l}^{+}, \omega_{l}^{-}}$replaced by 1 and $g_{\omega_{l}}^{(h)}$ replaced by $g^{(h)}$. 


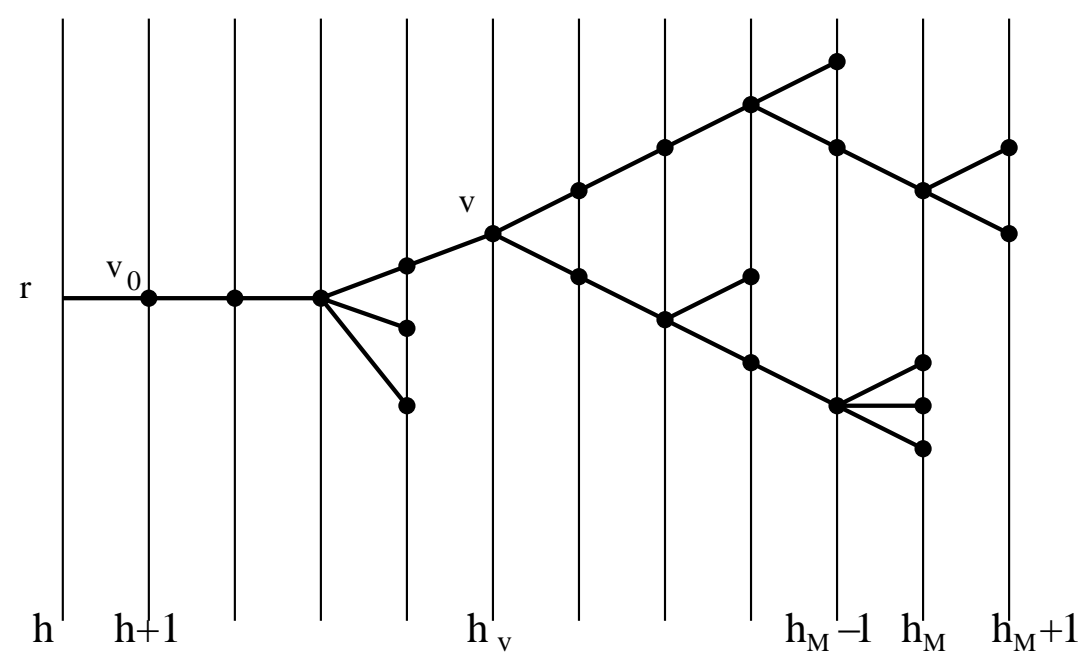

FIG. 2: A tree $\tau \in \mathcal{T}_{M ; h, n}$ with its scale labels.

As in the proof of Theorem 2, we get the bound

$$
\begin{gathered}
\frac{1}{\beta|\Lambda|} \int d \mathbf{x}_{1} \cdots d \mathbf{x}_{2 l}\left|W_{2 l, \underline{\rho}}^{(h)}\left(\mathbf{x}_{1}, \ldots, \mathbf{x}_{2 l}\right)\right| \leq \sum_{n \geq 1}|U|^{n} \sum_{\tau \in \mathcal{T}_{M ; h, n}} \sum_{\substack{\mathbf{P} \in \mathcal{P}_{\tau} \\
\left|P_{v_{0}}\right|=2 l}} \sum_{T \in \mathbf{T}} \int \prod_{l \in T^{*}} d\left(\mathbf{x}_{l}-\mathbf{y}_{l}\right) \cdot \\
\cdot\left[\prod_{i=1}^{n}\left|K_{v_{i}^{*}}\right|\right] \cdot\left[\prod_{v \text { not e.p. }} \frac{1}{s_{v} !} \max _{\mathbf{t}_{v}}\left|\operatorname{det} G^{h_{v}, T_{v}}\left(\mathbf{t}_{v}\right)\right| \prod_{l \in T_{v}} \| g^{(h)}\left(\mathbf{x}_{l}-\mathbf{y}_{l}\right)||\right]
\end{gathered}
$$

and, using the analogues of the estimates (3.92), (3.93) and (3.94), taking into account the new scaling of the propagator, we find that (B.17) can be bounded above by

$$
\sum_{n \geq 1} \sum_{\tau \in \mathcal{T}_{M ; h, n}} \sum_{\substack{\mathbf{P} \in \mathcal{P}_{\tau} \\\left|P_{v_{0}}\right|=2 l}} \sum_{T \in \mathbf{T}} C^{n}|U|^{n}\left[\prod_{v} \frac{1}{\text { not e.p. }_{\text {e. }} !} \gamma^{-h_{v}\left(s_{v}-1\right)}\right] .
$$

Using (3.96) we find that the latter expression can be rewritten as

$$
\sum_{n \geq 1} \sum_{\tau \in \mathcal{T}_{M ; h, n}} \sum_{\substack{\mathbf{P} \in \mathcal{P}_{\mathcal{T}} \\\left|P_{v_{0}}\right|=2 l}} \sum_{T \in \mathbf{T}} C^{n}|U|^{n} \gamma^{-h(n-1)}\left[\prod_{v \text { not e.p. }} \frac{1}{s_{v} !} \gamma^{-\left(h_{v}-h_{v^{\prime}}\right)(n(v)-1)}\right],
$$

where we remind the reader that $n(v)>1$ for any $\tau \in \mathcal{T}_{M ; h, n}$. Performing the sums over $T, \mathbf{P}$ and $\tau$ as in the proof of Theorem 2, we finally find

$$
\frac{1}{\beta|\Lambda|} \int d \mathbf{x}_{1} \cdots d \mathbf{x}_{2 l}\left|W_{2 l, \underline{\rho}}^{(h)}\left(\mathbf{x}_{1}, \ldots, \mathbf{x}_{2 l}\right)\right| \leq C|U|^{\max \{1, n-1\}},
$$

which is a special case of (3.37). The proof of the general case is completely analogous.

\section{APPENDIX C: GRAPHENE AS ASYMPTOTIC INFRARED MASSIVE $Q E D_{2+1}$}

In this Appendix we describe the relation between 2D graphene and a regularized version of euclidean $Q E D_{2+1}$ with a massive photon, massless dirac fermions and an ultraviolet cut-off. Let us first introduce the model of regularized $Q E D_{2+1}$ and let us next describe its connections with the graphene model described in this paper. 
We consider the following generating function for euclidean $Q E D_{2+1}$ :

$$
e^{\mathcal{W}_{L, a}(J, \phi)}=\int P(d \psi) P(d A) e^{\int d \mathbf{x}\left(e_{0} A_{\mu, \mathbf{x}} \bar{\psi}_{\mathbf{x}} \gamma_{\mu} \psi_{\mathbf{x}}+J_{\mu, \mathbf{x}} \bar{\psi}_{\mathbf{x}} \gamma_{\mu} \psi_{\mathbf{x}}+\phi_{\mathbf{x}} \bar{\psi}_{\mathbf{x}}+\bar{\phi}_{\mathbf{x}} \psi_{\mathbf{x}}\right)}
$$

where:

1. if $c$ is the speed of light, $\int d \mathbf{x}$ is a shorthand for $a^{3} c^{-1} \sum_{\mathbf{x} \in \Lambda_{a}}, a$ is the lattice spacing and $\Lambda_{a}$ is a periodic lattice of side $L c^{-1}$ in the time direction, of side $L$ in the two spatial directions, and with sites labelled by $x_{0}=n_{0} a c^{-1}, \vec{x}=\vec{n} a$, with $L a^{-1}$ integer and $n_{\mu}=0, \ldots, L a^{-1}-1, \mu=0,1,2$;

2. summation over repeated indices $\mu=0,1,2$ is understood;

3. $e_{0}$ is a constant, $J_{\mu}$ and $\phi$ are the external fields, and $\gamma_{\mu}$ are euclidean gamma matrices, satisfying $\left\{\gamma_{\mu}, \gamma_{\nu}\right\}=-2 \delta_{\mu \nu}$, and defined as

$$
\begin{aligned}
& \gamma_{0}=-i\left(\begin{array}{cc}
0 & \sigma_{0} \\
\sigma_{0} & 0
\end{array}\right), \quad \gamma_{1}=\left(\begin{array}{cc}
0 & \sigma_{2} \\
-\sigma_{2} & 0
\end{array}\right), \quad \gamma_{2}=\left(\begin{array}{cc}
0 & \sigma_{1} \\
-\sigma_{1} & 0
\end{array}\right) \\
& \text { with } \sigma_{\mu}, \mu=0,1,2 \text {, the Pauli matrices: } \\
& \qquad \sigma_{0}=\left(\begin{array}{cc}
1 & 0 \\
0 & 1
\end{array}\right), \quad \sigma_{1}=\left(\begin{array}{cc}
0 & 1 \\
1 & 0
\end{array}\right), \quad \sigma_{2}=\left(\begin{array}{cc}
0 & -i \\
i & 0
\end{array}\right) ;
\end{aligned}
$$

4. $\psi_{\mathbf{x}}$ is a 4-components Grassmann spinor of components $\psi_{\mathbf{x}, i}, i=1, \ldots, 4$; moreover, $\bar{\psi}_{\mathbf{x}}=\psi_{\mathbf{x}}^{+} \gamma_{0}$, with $\psi_{\mathbf{x}}^{+}$a Grassmann spinor of components $\psi_{\mathbf{x}, i}^{+}$;

5. let $\mathcal{D}$ be the set of space-time momenta $\mathbf{k}$ with $k_{0}=2 \pi c L^{-1}\left(m_{0}+\frac{1}{2}\right), \vec{k}=2 \pi L^{-1} \vec{m}$, with $m_{\mu}=0,1, \ldots, L a^{-1}-1, \mu=0,1,2$; if we define $\hat{\psi}_{\mathbf{k}, i}^{ \pm}=a^{3} c^{-1} \sum_{\mathbf{x} \in \Lambda_{a}} e^{\mp i \mathbf{k x}} \psi_{\mathbf{x}, i}$, the fermionic integration can be written as

$$
P(d \psi)=\frac{1}{\mathcal{N}}\left[\prod_{\mathbf{k} \in \mathcal{D}} \prod_{i=1}^{4} d \hat{\psi}_{\mathbf{k}, i}^{+} d \hat{\psi}_{\mathbf{k}, i}\right] \exp \left\{-\frac{Z}{L^{3} c^{-1}} \sum_{\mathbf{k} \in \mathcal{D}} \chi_{0}^{-1}(|\mathbf{k}|) \bar{\psi}_{\mathbf{k}} i \mathbf{k} \psi_{\mathbf{k}}\right\},
$$

where $\mathbf{k}=\gamma_{\mu} k_{\mu}, c$ is the speed of light, $Z$ is the wave function renormalization, $\mathcal{N}$ is a normalization constant and $\chi_{0}$ is the cut-off function introduced in Sec IIIB.

6. $A_{\mu, \mathbf{x}}$ is a euclidean gaussian boson field associated to the gaussian measure $P(d A)$ with covariance

$$
v_{\mu, \nu}(\mathbf{x}-\mathbf{y})=\delta_{\mu \nu} v(\mathbf{x}-\mathbf{y}) \equiv \delta_{\mu \nu} \frac{c}{L^{3}} \sum_{\mathbf{p} \in \mathcal{D}} e^{-i p_{0}(\mathbf{x}-\mathbf{y})} \frac{\chi_{0}(|\mathbf{p}|)}{\mathbf{p}^{2}+M^{2}}
$$

with $M>1$ the "photon mass".

Integrating out the gaussian boson field, we can rewrite:

$$
e^{\mathcal{W}_{L, a}(J, \phi)}=\int P(d \psi) e^{-\mathcal{V}(\psi)+\int d \mathbf{x} J_{\mu, \mathbf{x}} \bar{\psi}_{\mathbf{x}} \gamma_{\mu} \psi_{\mathbf{x}}+\int d \mathbf{x}\left(\phi_{\mathbf{x}} \bar{\psi}_{\mathbf{x}}+\bar{\phi}_{\mathbf{x}} \psi_{\mathbf{x}}\right)}
$$

where

$$
\mathcal{V}(\psi)=-\frac{e_{0}^{2}}{2} \int d \mathbf{x} d \mathbf{y}\left(\bar{\psi}_{\mathbf{x}} \gamma_{\mu} \psi_{\mathbf{x}}\right) v(\mathbf{x}-\mathbf{y})\left(\bar{\psi}_{\mathbf{y}} \gamma_{\mu} \psi_{\mathbf{y}}\right)
$$

The four dimensional version of the above model was studied in [19] by RG methods; the analysis (that can be repeated for the three dimensional model considered here without any relevant difference) is essentially identical to the one described in this paper for the 2D Hubbard model. 
Note in particular that, identifying the spinor $\hat{\psi}_{\mathbf{k}}$ with $\left(\hat{\Psi}_{\mathbf{k}, \sigma, 1,+}, \hat{\Psi}_{\mathbf{k}, \sigma, 2,+}, \hat{\Psi}_{\mathbf{k}, \sigma, 2,-}, \hat{\Psi}_{\mathbf{k}, \sigma, 1,-}\right)$, both the fermionic integration $P(d \psi)$ and the effective interaction $\mathcal{V}(\psi)$ are invariant under a number of symmetries, analogous to (4)-(8) of Lemma 1, i.e.,

$\left(4^{\prime}\right) \hat{\Psi}_{\left(k_{0}, k_{1}, k_{2}\right), \sigma, 1, \omega}^{ \pm} \rightarrow \hat{\Psi}_{\left(k_{0}, k_{2}, k_{1}\right), \sigma, 2, \omega}^{ \pm}, \hat{\Psi}_{\left(k_{0}, k_{2}, k_{1}\right), \sigma, 2, \omega}^{ \pm} \rightarrow(\mp i \omega) \hat{\Psi}_{\left(k_{0}, k_{1}, k_{2}\right), \sigma, 1, \omega}^{ \pm} ;$

$\left(5^{\prime}\right) \hat{\Psi}_{\mathbf{k}, \sigma, \rho, \omega}^{ \pm} \rightarrow \hat{\Psi}_{-\mathbf{k}, \sigma, \rho,-\omega}^{ \pm}, c \rightarrow c^{*}$, where $c$ is a generic constant appearing in $P(d \Psi)$ and/or in $\mathcal{V}(\psi)$;

$\left(6^{\prime}\right.$.a) $\hat{\Psi}_{\left(k_{0}, k_{1}, k_{2}\right), \sigma, 1, \omega}^{ \pm} \hat{\Psi}_{\left(k_{0},-k_{1}, k_{2}\right), \sigma, 2, \omega}^{ \pm}$

$\left(6^{\prime} . \mathrm{b}\right) \hat{\Psi}_{\left(k_{0}, k_{1}, k_{2}\right), \sigma, \rho, \omega}^{ \pm} \rightarrow \hat{\Psi}_{\left(k_{0}, k_{1},-k_{2}\right), \sigma, \rho,-\omega}^{ \pm} ;$

$\left(7^{\prime}\right) \hat{\Psi}_{\left(k_{0}, \vec{k}\right), \sigma, \rho, \omega}^{ \pm} \rightarrow i \hat{\Psi}_{\left(k_{0},-\vec{k}\right), \sigma, \rho,-\omega}^{\mp} ;$

$\left(8^{\prime}\right) \hat{\Psi}_{\left(k_{0}, \vec{k}\right), \sigma, \rho}^{ \pm} \rightarrow i(-1)^{\rho} \hat{\Psi}_{\left(-k_{0}, \vec{k}\right), \sigma, \rho}^{ \pm}$.

It is important to note that, in addition to the symmetries (4')-(8') above, $Q E D_{2+1}$ also admits extra symmetries, related to its relativistic invariance, which have no counterpart in the Hubbard model, e.g.,

$\left(9^{\prime}\right) \psi_{\mathbf{k}} \rightarrow e^{\frac{\theta}{4}\left[\gamma_{0}, \gamma_{1}\right]} \psi_{R_{\theta}^{-1} \mathbf{k}}, \bar{\psi}_{\mathbf{k}} \rightarrow \bar{\psi}_{R_{\theta}^{-1} \mathbf{k}} e^{-\frac{\theta}{4}\left[\gamma_{0}, \gamma_{1}\right]}$, where $R_{\theta} \mathbf{k}=\left(k_{0} \cos \theta-c k_{1} \sin \theta, k_{1} \cos \theta+\right.$ $\left.c^{-1} k_{0} \sin \theta, k_{2}\right)$. Note that in the limit $L, a^{-1} \rightarrow \infty$, there is no constraint on the choice of $\theta$, while for finite $L$ and $a$ we are forced to choose $\theta=\pi / 2$. The proof of the invariance of the model under the symmetry $\left(9^{\prime}\right)$ is a simple consequence of the remark that

$$
e^{-\frac{\theta}{4}\left[\gamma_{0}, \gamma_{1}\right]}\left(\gamma_{0}, \gamma_{1}, \gamma_{2}\right) e^{\frac{\theta}{4}\left[\gamma_{0}, \gamma_{1}\right]}=\left(\gamma_{0} \cos \theta-\gamma_{1} \sin \theta, \gamma_{1} \cos \theta+\gamma_{0} \sin \theta, \gamma_{2}\right)
$$

which implies that $\sum_{\mathbf{k} \in \mathcal{D}} \bar{\psi}_{\mathbf{k}} \mathbf{k} \psi_{\mathbf{k}}$ is invariant under $\left(9^{\prime}\right)$. In particular, if $\theta=\pi / 2$, in terms of the components $\hat{\Psi}_{\mathbf{k}, \rho, \sigma, \omega}$ of the spinor, (9') reads as follows:

$$
\begin{aligned}
& \hat{\Psi}_{\left(k_{0}, k_{1}, k_{2}\right), \sigma, \rho, \omega} \rightarrow \frac{1}{\sqrt{2}}\left(\sigma_{0}+i \sigma_{2}\right)_{\rho, \rho^{\prime}} \hat{\Psi}_{\left(c k_{1},-c^{-1} k_{0}, k_{2}\right), \sigma, \rho^{\prime}, \omega}, \\
& \hat{\Psi}_{\left(k_{0}, k_{1}, k_{2}\right), \sigma, \rho, \omega}^{+} \rightarrow \frac{1}{\sqrt{2}} \hat{\Psi}_{\left(c k_{1},-c^{-1} k_{0}, k_{2}\right), \sigma, \rho^{\prime}, \omega}^{+}\left(\sigma_{0}+i \sigma_{2}\right)_{\rho^{\prime}, \rho} .
\end{aligned}
$$

This symmetry also implies that the kernels of the quadratic part of the effective potentials have a special structure. In fact, repeating the proof of Lemma 2, using symmetries $\left(4^{\prime}\right)-\left(8^{\prime}\right)$, and if $\hat{W}_{2,\left(\rho_{1}, \rho_{2}\right), \omega}^{(h)}(\mathbf{k})$ is the kernel of the quadratic part of the effective action at scale $h$, we find the analogue of (3.62):

$$
\mathbf{k}^{\prime} \partial_{\mathbf{k}^{\prime}} \hat{W}_{2,\left(\rho_{1}, \rho_{2}\right), \omega}^{(h)}(\mathbf{0})=\left(\begin{array}{cc}
-i z_{h} k_{0} & \delta_{h}\left(i k_{1}-\omega k_{2}\right) \\
\delta_{h}\left(-i k_{1}-\omega k_{2}\right) & -i z_{h} k_{0}
\end{array}\right)_{\rho_{1}, \rho_{2}} .
$$

On the other hand, for $Q E D_{2+1}$ we also know that

$$
\sum_{\mathbf{k} \in \mathcal{D}} \hat{\Psi}_{\mathbf{k}, \sigma, \cdot, \omega}^{+}\left(\begin{array}{cc}
-i z_{h} k_{0} & \delta_{h}\left(i k_{1}-\omega k_{2}\right) \\
\delta_{h}\left(-i k_{1}-\omega k_{2}\right) & -i z_{h} k_{0}
\end{array}\right) \hat{\Psi}_{\mathbf{k}, \sigma, \cdot, \omega}
$$

must be invariant under (C.9), which implies $c z_{h}=\delta_{h}$, i.e., the speed of light is not renormalized.

The same proof shows that if, in relativistic notation, $\sum_{\mathbf{k}} \bar{\psi}_{\mathbf{k}} \mathbf{k}_{\mu} W_{\mu} \psi_{\mathbf{k}}$ is invariant under (4')(9'), then $W_{\mu}=C \gamma_{\mu}$, for some constant $C$. This is precisely the same as in four dimensional euclidean QED. Therefore, we can repeat step by step the construction in [19] and, in particular, we find that the following Ward Identity (WI) is valid:

$$
i Z e_{0} \mathbf{p}_{\mu}\left\langle j_{\mu, \mathbf{p}} ; \psi_{\mathbf{k}} \bar{\psi}_{\mathbf{k}-\mathbf{p}}\right\rangle=e\left[\left\langle\psi_{\mathbf{k}-\mathbf{p}} \bar{\psi}_{\mathbf{k}-\mathbf{p}}\right\rangle-\left\langle\psi_{\mathbf{k}} \bar{\psi}_{\mathbf{k}}\right\rangle\right]\left(1+H_{0}(\mathbf{k}, \mathbf{p})\right),
$$

where:

1. $\left\langle j_{\mu, \mathbf{p}} ; \psi_{\mathbf{k}} \bar{\psi}_{\mathbf{k}-\mathbf{p}}\right\rangle=\int d \mathbf{x} \int d \mathbf{z} e^{-i \mathbf{p}(\mathbf{z}-\mathbf{y})} e^{i \mathbf{k}(\mathbf{x}-\mathbf{y})}\left\langle j_{\mu, \mathbf{z}} ; \psi_{\mathbf{x}} \bar{\psi}_{\mathbf{y}}\right\rangle$, with

$$
\left\langle j_{\mu, \mathbf{z}} ; \psi_{\mathbf{x}} \bar{\psi}_{\mathbf{y}}\right\rangle=\left.\lim _{L, a^{-1} \rightarrow \infty} \frac{\partial^{3} \mathcal{W}_{L, a}(J, \phi)}{\partial \bar{\phi}_{\mathbf{x}} \partial \phi_{\mathbf{y}} \partial J_{\mu, \mathbf{z}}}\right|_{J=\phi=\bar{\phi}=0}
$$


similarly,

$$
\left\langle\psi_{\mathbf{x}} \bar{\psi}_{\mathbf{y}}\right\rangle=\frac{c}{L^{3}} \sum_{\mathbf{k} \in \mathcal{D}} e^{-i \mathbf{k}(\mathbf{x}-\mathbf{y})}\left\langle\psi_{\mathbf{k}} \bar{\psi}_{\mathbf{k}}\right\rangle=\left.\lim _{L, a^{-1} \rightarrow \infty} \frac{\partial^{2} \mathcal{W}_{L, a}(J, \phi)}{\partial \bar{\phi}_{\mathbf{x}} \partial \phi_{\mathbf{y}}}\right|_{J=\phi=\bar{\phi}=0}
$$

2. $e=e_{0}-c_{+} e_{0}^{3}+O\left(e_{0}^{5}\right)$, with $c_{+}$a suitable constant;

3. the correction $H_{0}(\mathbf{k}, \mathbf{p})$ is such that, for momenta $\mathbf{k}, \mathbf{p}, \mathbf{k}-\mathbf{p}$ all on the same scale $h$ (i.e, all belonging to the support of $f_{h}$, for some finite $h \leq 0$ )

$$
\left|H_{0}(\mathbf{k}, \mathbf{p})\right| \leq C|e| \gamma^{\theta h},
$$

for some $0<\theta<1$.

Note that the above WI differs from the formal WI obtained by neglecting the ultraviolet cut-off, because of the presence of the renormalized charge $e=e_{0}-c_{+} e_{0}^{3}+O\left(e_{0}^{5}\right)$ and of the correction $H_{0}(\mathbf{k}, \mathbf{p})$.

There is a strong connection between the above model and the Hubbard model. Indeed from (3.120) we know that

$$
S(\mathbf{x}-\mathbf{y})=S^{(1)}(\mathbf{x}-\mathbf{y})+\sum_{\omega= \pm} e^{-i \vec{p}_{F}^{ \pm}(\vec{x}-\vec{y})} S_{\omega}^{(\leq 0)}(\mathbf{x}-\mathbf{y})
$$

where $S_{\omega}^{(\leq 0)}(\mathbf{x}-\mathbf{y})$ is given by the sum in the second line of (3.118) restricted to $h \leq 0$, and $\left|S^{(1)}(\mathbf{x}-\mathbf{y})\right| \leq C|\mathbf{x}-\mathbf{y}|^{-2-\theta}$ for $|\mathbf{x}-\mathbf{y}| \geq 1$; this means that, for large distances, $S^{(1)}$ is asymptotically negligible with respect to $S_{\omega}^{(\leq 0)}(\mathbf{x}-\mathbf{y})$.

By (2.10) and the construction in Sections IIIC and IIID, we expect that the Grassmann spinor $\left(\hat{\Psi}_{\mathbf{k}, \sigma, 1,+}^{(\leq 0)}, \hat{\Psi}_{\mathbf{k}, \sigma, 2,+}^{(\leq 0)}, \hat{\Psi}_{\mathbf{k}, \sigma, 2,-}^{(\leq 0)}, \hat{\Psi}_{\mathbf{k}, \sigma, 1,-}^{(\leq 0)}\right)$ plays the same role as the spinor $\psi_{\mathbf{k}}$ in the $Q E D_{2+1}$ model. In order to make this intuition precise, it is convenient to combine $S_{ \pm}^{(\leq 0)}(\mathbf{x}-\mathbf{y})$ in the following matrix

$$
\mathcal{G}(\mathbf{x}-\mathbf{y})=\left(\begin{array}{cc}
0 & S_{+}^{(\leq 0)}(\mathbf{x}-\mathbf{y}) \\
S_{-}^{(\leq 0) T}(\mathbf{x}-\mathbf{y}) & 0
\end{array}\right),
$$

where $S_{\omega}^{(\leq 0) T}$ is the transpose of $S_{\omega}^{(\leq 0)} \cdot \mathcal{G}(\mathbf{x}-\mathbf{y})$ will play the same role as the correlation $\left\langle\psi_{\mathbf{x}} \bar{\psi}_{\mathbf{y}}\right\rangle$ defined in (Cl15), in a sense to be made precise below. Similarly, the role of $\left\langle j_{\mu, \mathbf{z}} ; \psi_{\mathbf{x}} \bar{\psi}_{\mathbf{y}}\right\rangle$ will be played by the correlation $S_{2,1 ; \mu}(\mathbf{z} ; \mathbf{x}, \mathbf{y}), \mu=0,1,2$, defined as

$$
S_{2,1 ; \mu}(\mathbf{z} ; \mathbf{x}, \mathbf{y})_{\rho, \rho^{\prime}}=\left\langle\mathbf{T}\left\{\Psi_{\mathbf{x}, \sigma, \rho}^{-} \Psi_{\mathbf{y}, \sigma, \rho^{\prime}}^{+} \Psi_{\mathbf{z}, \sigma,}^{+}, \sigma_{\mu} \Psi_{\mathbf{z}, \sigma, .}^{-}\right\}\right\rangle-\left\langle\mathbf{T}\left\{\Psi_{\mathbf{x}, \sigma, \rho}^{-} \Psi_{\mathbf{y}, \sigma, \rho^{\prime}}^{+}\right\}\right\rangle \cdot\left\langle\Psi_{\mathbf{z}, \sigma, \cdot}^{+} \sigma_{\mu} \Psi_{\mathbf{z}, \sigma, \sigma,}^{-}\right\rangle .
$$

By an analysis similar to the one in Section IIID, we get

$$
S_{2,1 ; \mu}(\mathbf{z} ; \mathbf{x}, \mathbf{y})=S_{2,1, \mu}^{(1)}(\mathbf{z} ; \mathbf{x}, \mathbf{y})+S_{2,1 ; \mu}^{+}(\mathbf{z} ; \mathbf{x}, \mathbf{y})+S_{2,1 ; \mu}^{-}(\mathbf{z} ; \mathbf{x}, \mathbf{y}),
$$

where the first term is asymptotically negligible with respect to the last two for large distances. The terms $S_{2,1 ; \mu}^{+}(\mathbf{z} ; \mathbf{x}, \mathbf{y})$ and $S_{2,1 ; \mu}^{-}(\mathbf{z} ; \mathbf{x}, \mathbf{y})$ correspond to contributions to the correlation function coming from the infrared integration, whose computation requires, as in Sections IIC and IIID the decomposition of the infrared field into the sum of quasi-particle fields indexed by $\omega= \pm$ and supported, in momentum space, around the two different Fermi points $\vec{p}_{F}^{\omega}$. By the compact support properties of the infrared fields, in the terms contributing to $S_{2,1 ; \mu}^{ \pm}(\mathbf{z} ; \mathbf{x}, \mathbf{y})$, the quasi-particle indeces corresponding to the fields located at $\mathbf{z}$ are the same, and will be denoted by $\omega_{\mathbf{z}}$; similarly, the quasi-particle indeces corresponding to the fields located at $\mathbf{x}$ and $\mathbf{y}$ are the same, and will be denoted by $\omega_{\mathbf{x y}}$. Finally, $S_{2,1 ; \mu}^{+}(\mathbf{z} ; \mathbf{x}, \mathbf{y})$ is defined as the sum of all the contributions such that $\omega_{\mathbf{z}}=\omega_{\mathbf{x y}}$, while $S_{2,1 ; \mu}^{-}(\mathbf{z} ; \mathbf{x}, \mathbf{y})$ corresponds to the terms with $\omega_{\mathbf{z}}=-\omega_{\mathbf{x y}}$. By construction, $S_{2,1 ; \mu}^{+}(\mathbf{z} ; \mathbf{x}, \mathbf{y})$ can be written as a sum over two terms:

$$
S_{2,1 ; \mu}^{+}(\mathbf{z} ; \mathbf{x}, \mathbf{y})=\sum_{\omega= \pm} e^{-i \vec{p}_{F}^{\omega}(\vec{x}-\vec{y})} S_{2,1 ; \mu, \omega}^{+}(\mathbf{z} ; \mathbf{x}, \mathbf{y})
$$


and we can combine such terms in a single matrix

$$
\Gamma_{\mu}(\mathbf{z} ; \mathbf{x}, \mathbf{y})=\frac{1}{(\beta|\Lambda|)^{2}} \sum_{\mathbf{k}, \mathbf{p}} e^{i \mathbf{p z}} e^{-i \mathbf{k x}} e^{i(\mathbf{k}-\mathbf{p}) \mathbf{y}} \hat{\Gamma}_{\mu}(\mathbf{p}, \mathbf{k})=\left(\begin{array}{cc}
0 & \hat{S}_{2,1 ; \mu,+}^{+}(\mathbf{z} ; \mathbf{x}, \mathbf{y}) \\
\hat{S}_{2,1 ; \mu,-}^{+, T}(\mathbf{z} ; \mathbf{x}, \mathbf{y}) & 0
\end{array}\right) .
$$

It is clear from the multiscale construction of these correlation functions that, with a proper choice of the parameters, such matrices are asymptotically close to the Schwinger function of the $Q E D_{2+1}$ model seen above, as explained by the following theorem, which is, in fact, a corollary of the analysis in the previous sections and of a finite dimensional fixed point argument.

Theorem 3. Let $U$ and $e_{0}$ be small enough. It is possible to choose $Z$ and $c$ in (C.1)-(C.5) as functions of $U, e_{0}, M$ and $v(\mathbf{0})$, so that, if $\mathbf{k}, \mathbf{p}, \mathbf{k}-\mathbf{p}$ are all on the same scale $h$ (i.e., if $\left.a_{0} \gamma^{h-1} \leq|\mathbf{k}|,|\mathbf{p}|,|\mathbf{k}-\mathbf{p}| \leq a_{0} \gamma^{h+1}, h \leq 0\right)$,

$$
\begin{aligned}
& \mathcal{G}(\mathbf{k})=\left\langle\psi_{\mathbf{k}} \bar{\psi}_{\mathbf{k}}\right\rangle\left(1+O\left(\gamma^{\theta h}\right)\right), \\
& \Gamma_{\mu}(\mathbf{k}, \mathbf{p})=Z_{\mu}\left\langle j_{\mathbf{p}}^{\mu} ; \psi_{\mathbf{k}} \bar{\psi}_{\mathbf{k}+\mathbf{p}}\right\rangle\left(1+O\left(\gamma^{\theta h}\right)\right),
\end{aligned}
$$

where $Z_{0}, Z_{1}, Z_{2}$ in (C.23) are suitable constants, depending on $U, e_{0}, M$ and $v(\mathbf{0})$ and $0<\theta<1$.

Theorem 3 says that, by choosing the wave function renormalization and the velocity of light in the $Q E D$ model as suitable functions of $U, e_{0}, M$ and $v(\mathbf{0})$, its two point Schwinger functions coincide with the ones of the Hubbard model, up to corrections which are negligible at small momenta. With this choice of $Z$ and $c$, the vertex functions of $Q E D_{2+1}$ are asymptotically proportional to those of the Hubbard model, provided that the renormalizations $Z_{\mu}$ are properly chosen. Note that, while in a relativistic QFT $Z_{\mu}$ is $\mu$-independent, here it is not [14], the symmetry (9') being broken by the underlying lattice; however, one can check, by arguments similar to the ones used in the proof of Lemma 2, that the lattice symmetries imply that the renormalizations $Z_{\mu}$ are still diagonal in $\mu$ : note, in fact, that in principle the r.h.s. of (C,23) could be of the form

$$
\sum_{\nu} Z_{\mu, \nu}\left\langle j_{\mathbf{p}}^{\nu} ; \psi_{\mathbf{k}} \bar{\psi}_{\mathbf{k}+\mathbf{p}}\right\rangle\left(1+O\left(\gamma^{\theta h}\right)\right)
$$

but, remarkably, $Z_{\mu, \nu}$ turns out to be diagonal.

Theorem 3 implies that the Schwinger functions of the 2D Hubbard model on the honeycomb lattice obey to a Ward Identity analogous to (C.12), as it follows by combining (C,12) with (C|22) (Cl23), see 14]. This is true not only in the free case $U=0$ (in which case the WI can be verified by a simple explicit computation) but also, remarkably, in the interacting case. Note that, with respect to the WI for QED, the WI for the Hubbard model is modified by the presence of some proportionality constants, which take into account both the relativistic renormalization of the charge and the fact that the Hubbard model breaks some relativistic symmetries.

Let us conclude by remarking that, while here the WI can be proved a posteriori of the construction of the correlation functions, in the presence of Coulomb interactions the validity of an analogous WI is believed to play a crucial role in the construction of the model itself, as in one dimension [18]: in fact, in that case, the interparticle interaction becomes marginal in a RG sense [11] and the presence of WIs is a key ingredient in the control of the flow of the beta function equation, as in QED or in the Luttinger model.

[1] G. Benfatto, G. Gallavotti: Perturbation theory of the Fermi surface in a quantum liquid. A general quasiparticle formalism and one-dimensional systems, Jour. Stat. Phys. 59, 541-664 (1990).

[2] G. Benfatto, G. Gallavotti: Renormalization Group, Princeton University Press (1995).

[3] G. Benfatto, A. Giuliani and V. Mastropietro: Fermi liquid behavior in the 2D Hubbard model at low temperatures, Ann. Henri Poincaré 7, 809-898 (2006). 
[4] G. Benfatto, G. Gallavotti, A. Procacci, B. Scoppola: Beta function and Schwinger functions for a many fermions system in one dimension. Anomaly of the fermi surface, Comm. Math. Phys. 160, 93-171 (1994).

[5] G. Benfatto, V. Mastropietro: Renormalization group, hidden symmetries and approximate Ward identities in the XYZ model, Rev. Math. Phys. 13, 1323-1435 (2001).

[6] A. H. Castro Neto, F. Guinea, N. M. R. Peres, K. S. Novoselov, A. K. Geim: The electronic properties of graphene, Rev. Mod. Phys., in press.

[7] M. Disertori, V. Rivasseau: Interacting Fermi Liquid in Two Dimensionsat Finite Temperature. Part I and II, Comm. Math. Phys. 215, 251-290 and 291-341 (2000).

[8] J. Feldman, H. Knörrer and E. Trubowitz: A Two Dimensional Fermi Liquid, Comm. Math. Phys 247, 1-319 (2004).

[9] K. Gawedski, A. Kupiainen: Gross-Neveu model through convergent perturbation expansions, Comm. Math. Phys. 102, 1-30 (1985).

[10] A. Giuliani: Ground state energy of the low density Hubbard model: An upper bound, J. Math. Phys. 48, 023302 (2007).

[11] J. Gonzalez, F. Guinea and M. A. H. Vozmediano: Non-Fermi liquid behavior of electrons in the half-filled honeycomb lattice (A renormalization group approach), Nucl. Phys. B 424, 595-618 (1994).

[12] J. Gonzalez, F. Guinea and M. A. H. Vozmediano: Electron-electron interactions in graphene sheets, Phys. Rev. B 63, 134421 (2001).

[13] G. Gentile, V. Mastropietro: Renormalization group for one-dimensional fermions. A review on mathematical results. Renormalization group theory in the new millennium, III, Phys. Rep. 352, 273-437 (2001).

[14] A. Giuliani, V. Mastropietro: Rigorous construction of ground state correlations in graphene: renormalization of the velocities and Ward Identities, arXiv:0901.4867.

[15] A. Lesniewski: Effective action for the Yukawa 2 quantum field theory, Comm. Math. Phys. 108, 437-467 (1987).

[16] E. H. Lieb: Two Theorems on the Hubbard Model, Phys. Rev. Lett. 62, 1201-1204 (1989). Errata 62, 1927 (1989).

[17] E. H. Lieb, R. Seiringer and J. P. Solovej: Ground-state energy of the low-density Fermi gas, Phys. Rev. A 71, 053605-1-13 (2005).

[18] V. Mastropietro: Non-Perturbative Renormalization, World Scientific (2008).

[19] V. Mastropietro: Renormalization Group and Ward Identities for infrared QED4, J. Math. Phys. 48, 102303 (2007).

[20] K. S. Novoselov, A. K. Geim, S. V. Morozov, D. Jiang, Y. Zhang, S. V. Dubonos, I. V. Grigorieva, A. A. Firsov: Electric Field Effect in Atomically Thin Carbon Films, Science 306666 (2004).

[21] W. Pedra, M. Salmhofer: Determinant bounds and the Matsubara UV problem of many-fermion systems, Comm. Math. Phys. 282 797-818 (2008).

[22] M. Salmhofer: Renormalization: An Introduction, Springer (1999).

[23] R. Seiringer and J. Yin: Ground state energy of the low density Hubbard model, J. Stat. Phys. 131, 1139-1154 (2008).

[24] G. W. Semenoff: Condensed-Matter Simulation of a Three-Dimensional Anomaly, Phys. Rev. Lett. 53, 2449-2452 (1984).

[25] P. R. Wallace: The Band Theory of Graphite, Phys. Rev. Lett. 71, 622-634 (1947). 